\title{
3D Modelling and monitoring of denervated muscle under Functional Electrical Stimulation treatment and associated bone structural changes
}

Paolo Gargiulo

Department of. Science, Education and Innovation, Landspitali University Hospital and Department of. Biomedical Engineering, University of Reykjavik, Reykjavik, Iceland

\begin{abstract}
A novel clinical rehabilitation method for patients who have permanent and non recoverable muscle denervation in the legs was developed in the frame of the European Project RISE. The technique is based on FES and the project results shows, in these severely disabled patients, restoration of muscle tissue and function. This study propose novel methods based on image processing technique and medical modelling to monitor growth in denervated muscle treated with FES. Geometrical and structural changes in muscle and bone are studied and modelled. Secondary effects on the bone mineral density produced by the stimulation treatment and due the elicited muscle contraction are also investigated.

The restoration process in DDM is an important object of discussion since there isn't yet a complete understanding of the mechanisms regulating growth in denervated muscle. This study approaches the problem from a macroscopic point of view, developing 3-dimensional models of the whole stimulated muscles and following changes in volume, geometry and density very accurately. The method is based on the acquisition of high resolution Spiral CT scans from patients who have long-term flaccid paraplegia and the use of special image processing tools allowing tissue discriminations and muscle segmentation. Three patients were measured at different points of time during 4 years of electrical stimulation treatment.

In this study is quantitatively demonstrated the influences of FES treatment on the different quadriceps bellies. The rectus femoris muscle is positioned in the middle of the quadriceps and responds (in general) better to stimulation. In a patient with abundant adipose tissue surrounding the quadriceps, rectus femoris almost doubled the volume during the FES treatment while in the other bellies the changes measured were minimal. The analysis of the density shows clearly a restoration of the muscular structure in the growing muscle. The remarkable increase of muscle mass and the improved quality of the muscle tissue was observed and measured on whole muscle volume. The 3-dimensional approach proposed in this work allows the geometrical changes in denervated muscle to be measured. Central lines in rectus femoris are calculated during the treatment and the relative curvature indexes are used to quantify differences between data sets. The result shows a correlation between degeneration status and changes in shape; the differences in curvature between control and denervated muscle diminish with the growth.

Furthermore, bone mineral density is measured on the femur to monitor the structural changes induced by the current flow and to evaluate the effect on the patella bone density due to the quadriceps contraction. Though the evidences aren't yet statistically relevant a kind of structural change in the femur is measured as secondary effect from the muscle stimulation. Beside, the force applied on the patella through the elicited contraction seems also to have a beneficial effect on bone density. Indeed the mineral loss in the patella is remarkably slowed down.
\end{abstract}

Key Words: 3D Modelling and monitoring, , bone structural changes, skeletal muscle, lower motor neuron denervation, home based Functional Electrical Stimulation, h-b FES 
3D Modelling and monitoring of denervated muscle under FES

European Journal Translational Myology - Basic Applied Myology 2011; 21: 31-94

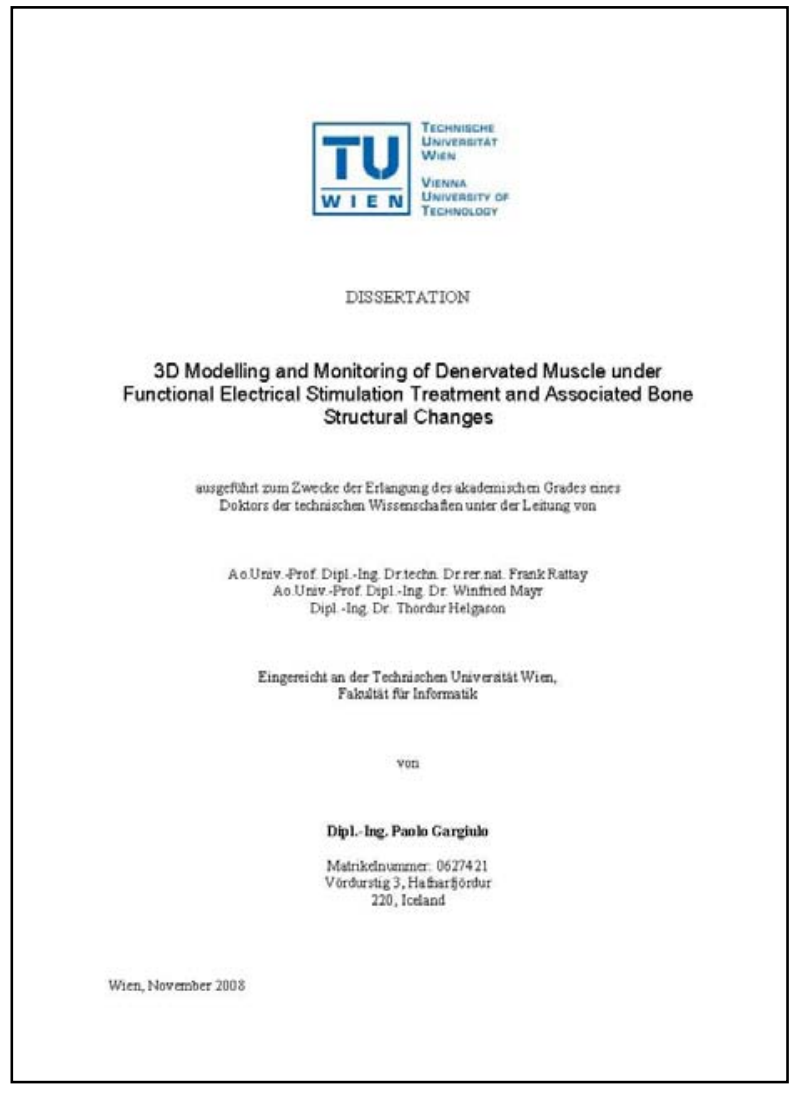

\section{INTRODUCTION}

1.1 FES: history and applications

1.1.1 FES in denervated muscle

1.2 RISE project

1.3 Medical modelling

1.3.1 Medical Modelling in Iceland

1.3.2 Modelling FES

1.4 Aim of the study

2. DATA, PROCESSING AND PATIENTS

2.1 Data acquisition

2.1.1 Spiral- Helical CT

2.1.2 Image representation

2.1.3 Use of Ct numbers for tissue characterisation

2.1.4 Acquisition parameters and scanning protocol

2.1.5 Calibration

2.2 Image processing tools

2.2.1 Segmentation techniques

2.3 Patients and compliance

\section{PUBLICATIONS}

3.1 Morphological changes in rectus femoris muscle: advanced image processing technique and 3 dimensional visualization to monitor denervated and degenerated muscles treated with functional electrical stimulation

3.2 Restoration of muscle volume and shape Induced by electrical stimulation of denervated degenerated muscles: qualitative and quantitative measurement of changes in rectus femoris using computer tomography and image segmentation

\section{MODELLING AND ANALYSIS OF RECTUS} FEMORIS MUSCLE

4.1 Introduction

4.2 Segmentation and 3-D visualization

4.3 Measurement of volume and density

4.3.1 Measurement of volume

4.3.2 Measurement of density

4.4 Muscle tissue analysis

4.4.1 Profile line method

4.4.2 Profile line results

4.5 Geometrical changes in rectus femoris

4.5.1 Central line method

4.5.2 Curvature index

4.6 Modelling muscle fiber direction

4.6.2 Denervated fiber direction: density tracker

4.6.3 Implementation: central line method

\section{MODELLING AND ANALYSIS OF QUADRICEPS MUSCLE}

5.1 Introduction

5.2 Segmentation of quadriceps muscle

5.3 Measurement of volume and density

5.3.1 Measurement of volume

5.3.2 Measurement of density distribution

5.3.3 Muscle tissue structure analysis

5.4 Monitoring of muscle and fat

6. BONE STRUCTURAL CHANGES AND MINERAL DENSITY DISTRIBUTION IN THE PATELLA

6.1 Introduction

6.2 Changes of bone density in femur bone as consequences of FES treatment on the quadriceps muscle

6.3 Modelling patella bone

6.3.1 Patella segmentation and reslicing

6.3.2 Conversion of HU values in bone mineral density

6.4 Bone mineral density measurement on the patella

7. DISCUSSION

7.1 Use of the results and future work

8. LITERATURE

9. Appendix

\section{INTRODUCTION}

\subsection{FES: history and applications}

Functional Electrical Stimulation (FES) is a part of the broader field of electrotherapy. The term was introduced relatively late in the history of FES by Moe and Post [1]. The natural electricity in the body carries signals back and forth between the central and peripheral nervous systems. The motor signals which start in the brain carry the electrical commands that cause voluntary muscles contraction. Sensory signals go in the opposite direction; they start at a limb, the trunk, or the head and carry electrical messages about touch, pressure or pain back to the brain. Injury or disease in the nervous system can interrupt the normal communication between the central and peripheral 


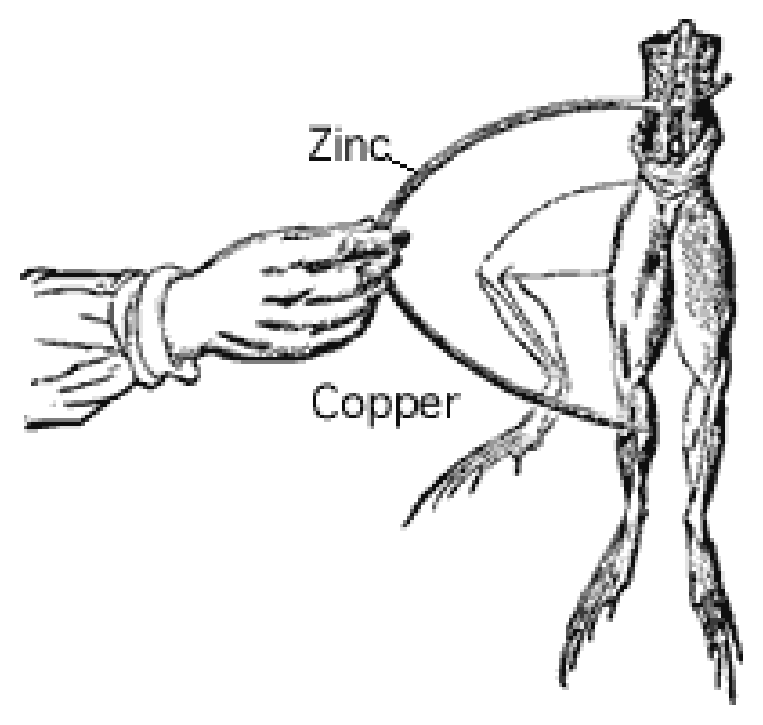

Fig 1 Galvani experiment

nervous systems. The result may be muscle weakness or paralysis.

Functional electrical stimulation (FES) is the method in which electrical stimulation is used to supply, restore or improve lost or impaired function of the body due to neurological disorders and peripheral nerve damage caused by spinal cord injury, stroke, traumatic brain injury or neurological disease.

The first known, remarkable experiment for functional electrical stimulation was the frog's leg experiment described by in Luigi Galvani 1791 [2]. In this experiment Galvani connected nerve and muscle of a frog's leg with an arc consisting of two different metals: zinc and copper (Fig. 1). The contact voltage elicited by this setup depolarised the contacted nerve and consequentially caused a contraction of the muscles. With this experiment Galvani could show the connection between electrical stimulation of the nerve and the contraction of muscles innervated by it.

The development of FES follows theoretical and technological breakthroughs in electricity and magnetism since clinical applications of this principle were always closely connected to the technological progress. The first and still today most developed FES application is the artificial heart pacemaker [3]. In 1932 Dr. Albert Hyman described an "artificial pacemaker" that he had used with experimental animals to resuscitate the "arrested" heart. About that time, the Australian physician Mark C. Lidwill, along with the physicist Major Edgar Booth, built the first portable pacemaking unit. It was demonstrated in 1931, while Hyman developed his device in 1930-31. Lidwill's apparatus had one pole applied to the skin and another into the appropriate cardiac chamber [4]. Later on, the Hyman device was successfully used in patients. Furman and Schwedel treated a patient 96

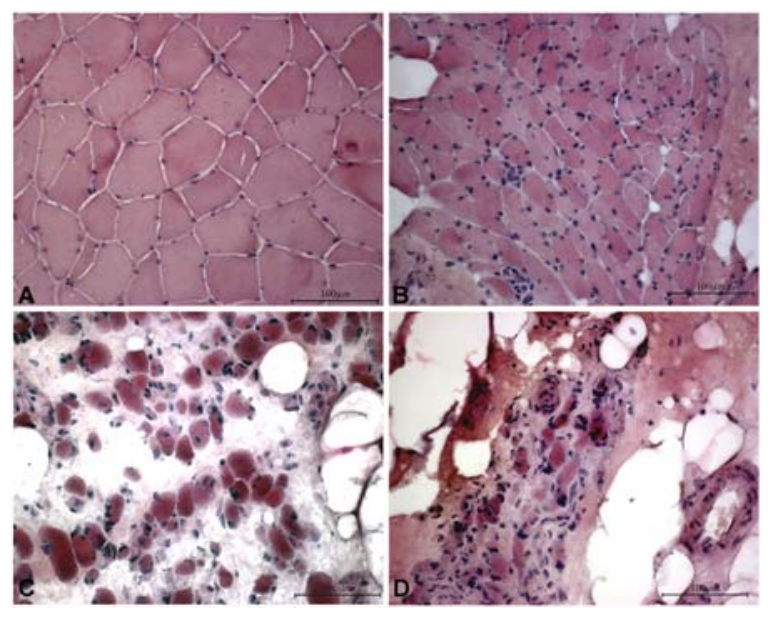

Fig 2 Muscle biopsy morphometry. A, Normal; B, 0.9-year denervation; $C$, 3.3-year denervation; D, 8.7-year denervation; Courtesy of Prof. Ugo Carraro - Padua Translational Myology Lab

days with a pacemaker in 1958. Because of the size of this pacemaker, it had to be carried on a trolley in front of the patient. The stimuli were applied trough percutaneous electrodes. A few months later the first fully implantable pacemaker was introduced. Nowadays far more than 500.000 patients per year are treated with cardiac pacemakers [5].

Most of FES clinical application are based on the principle that the electrical stimulus excite-depolarize nerve membranes or in general neural structures. Based on this principle are most developed, contemporary clinical applications of FES:

- Cochlear implant, stimulation of auditory nerve in the cochlea. [6]

- Drop-foot stimulator, stimulation of the peroneal nerve. [7]

- Hand disabilities, stimulation to support and/or restore reaching and grasping. [8,9]

- Phrenic stimulation, stimulation of the phrenic nerves to restore the function of the respiratory system [10]

FES is also assessed in other clinical applications such as: Bladder \& bowel control, treatment of pressure sores and treating of osteoporosis.

\subsubsection{FES in denervated muscle}

Practically all established clinical FES applications are based on the direct excitation of neural structures. In the case of absence of the neuromuscular junction caused by the damage of the motor units the muscle becomes denervated. The nerve-muscle connection is broken in denervated muscle and contraction can be elicited with FES by depolarizing directly the cellular membrane of each single muscle fiber. This means that the stimulation isn't selective, the muscle fatigues and gets weak very quickly because of the absence of branching nerves distributing the action potential to the 


\section{D Modelling and monitoring of denervated muscle under FES}

European Journal Translational Myology - Basic Applied Myology 2011; 21: 31-94

muscle fibers. The electrical membrane excitability strongly depends on the state of degeneration or restoration of the muscle cell, in any case excitability of denervated muscle membrane is much lower than in nerve cell and requires more energy.

The human skeletal muscle undergoes three phases during long-term denervation (Fig. 2):

i) Atrophy (up to 2-year SCI)

ii) Severe atrophy (3-5 years $\mathrm{SCI})$

iii) Degeneration (from 3-year SCI)

With the time, from twelve to eighteen months from the injury, myofiber degeneration is followed by progressive myonecrosis and by replacement with fat and fibrous connective tissue. The severe atrophy has secondary effects that include an increased propensity for developing pressure sores and osteoporosis.

From structural point of view, the lack of innervation causes severe alterations of fiber properties such as a progressive disarrangement of internal ultrastructure accompanied by functional impairment and followed by complete degeneration [11]. Some published studies demonstrate in both animal and clinical experiments that functional reactivation of denervated muscles by FES is possible, meaning that the contractile capability of the muscle isn't completely compromised even after long denervation $[12,13,14]$.

Anyway no efficient clinical treatment was available to alleviate or prevent the muscle atrophy that results from long-standing denervation until Dr. Kern with his team demonstrated the feasibility of reversing the degenerative status of the quadriceps muscle using intensive regime of direct stimulation through largearea electrodes placed over the skin of the anterior thigh. [15].

\subsection{RISE project}

Based on the idea and the empirical experience that denervated degenerated muscle can be recovered, in November 2001 the European funded project RISE started with the aim of establishing a novel clinical rehabilitation method for patients who have permanent and non recoverable muscle denervation in the legs. The technique based on Functional Electrical Stimulation (FES) aim to restore muscle tissue, muscle function and the ability to rise in these severely disabled patients.

Rehabilitative centres in Vienna (Austria), Heidelberg (Germany), Hamburg (Germany), Tuebingen (Germany), Reykjavik (Iceland) and Vicenza (Italy) specialised in SCI have been recruiting voluntary patients for the clinical trial.

The RISE project reached his goal with a multidisciplinary approach; developing the technologies to stimulate denervated muscle, developing a clinical trial with over 20 voluntary patients, making animal experiments and researching the restoration process by physiological, histological histochemical and biochemical analytical techniques.

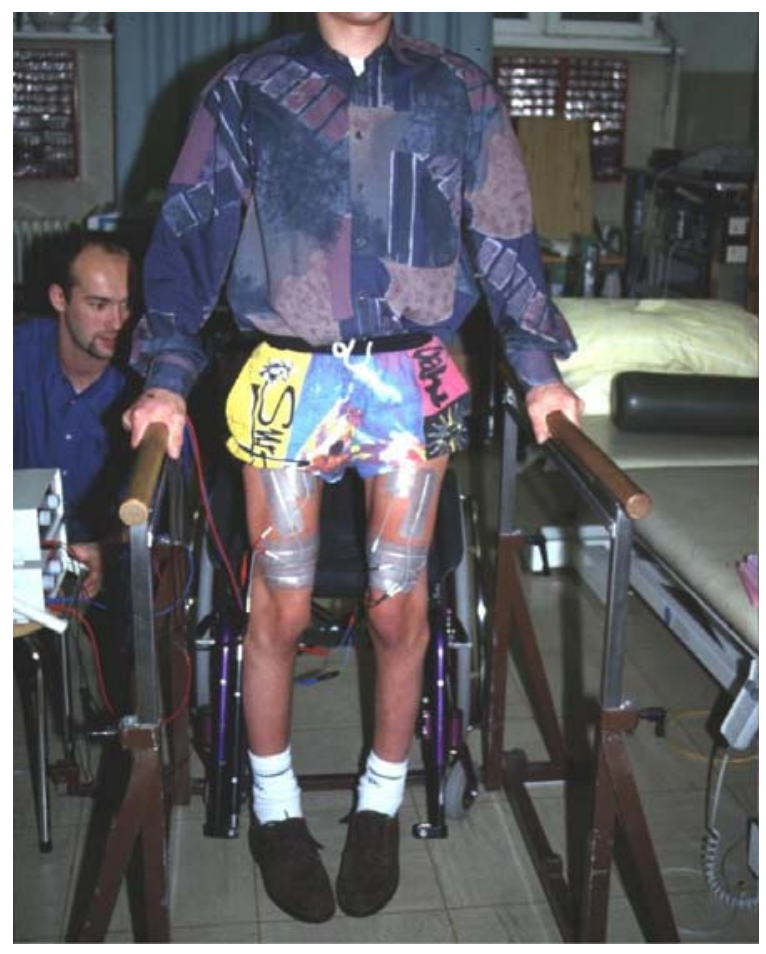

Fig 3 Standing exercise in a compliant patient participating the RISE clinical trial

As results of the RISE project appropriate technological solution were developed to allow muscle growth during clinical trial as well associate technology for animal experiment. A tremendous amount of research work was produced in the project frame and many remarkable multidisciplinary studies published in international journal.

Most of the basic scientific knowledge concerning denervated muscle and their treatment using FES is built during the RISE project thanks to striking research work done on animal models. Degeneration in long term denervated muscle fibre can be reversed; experiments on rats demonstrated the survival of residual myogenic activity in the denervated untreated muscle and a substantial increase of this activity after severe injury of the muscle [16].

Effects of long-term denervation on skeletal muscle and physiological and morphological changes induced by chronic electrical stimulation were studied on rabbits. The results show the restoration of the mass and contractile function of rabbit tibialis anterior (TA) muscles that had undergone atrophy as a result of prolonged denervation. [17] .

Remarkable also the studies on human muscle atrophy in long-lasting spastic paraplegia which demonstrate that the difference in average size of muscle fibers between long-term and mid-term paralyzed leg muscles is very small, suggesting that there are no 


\section{D Modelling and monitoring of denervated muscle under FES}

European Journal Translational Myology - Basic Applied Myology 2011; 21: 31-94

upper-time limits to begin a training program based on functional electrical stimulation [18] .

Beside research work on the ultra structural proprieties of stimulated denervated muscles shows that recovery of muscle fiber resembles some aspects of normal embryonic and postnatal muscle differentiation [19]. Anyway the results from the clinical and experimental work are still the most important; indeed the clinical trial demonstrate that electrical stimulation with exponential current can slow down atrophy of the DDM in human and modulated long-term training program restore function up to supported standing (Fig. 3). FES stimulation induced surprising recovery of muscle structure, mass, and force even in patients whose muscles had been denervated for prolonged periods before the beginning of FES training [20, 21 22].

The effectiveness of electrical stimulation protocols of DDM was demonstrated by measuring contraction force. Measurements by CT-scans reveal high variation in the increase of thigh muscle cross-sectional area during the first years of functional electrical stimulation (FES) [23].

To allow the clinical trial, proper stimulator device were developed to elicit functionally usable contractions in denervated muscles via surface electrodes. Indeed commercially available stimulation devices are only able to slow down atrophy or maintain the muscle during recovery after non permanent denervation. Most of the stimulators are only able to generate pulse widths of 300,200 or 100 microseconds ( $\mu \mathrm{sec}$ ) and much shorter pulses with about $1 \mathrm{~ms}$ pulse width but nothing in between. Beside the EU regulation for stimulators limits the output energy to $300 \mathrm{~mJ}$ per impulse which is not enough to elicit contraction of denervated and degenerated muscle fibers.

The developed stimulator activate denervated skeletal muscles via special customised surface electrodes using a biphasic long-duration impulses with a pulse width between 10 and $300 \mathrm{~ms}$ for excitation of the denervated muscle fibres and amplitudes of up to \pm 100 $\mathrm{V}$ and $\pm 250 \mathrm{~mA}$ which are sufficient for stimulating long term denervated muscles through the surrounding connective tissue.

The prototype device is designed making special attentions on patient security and safety issue; controls are limited to the user and are isolated from the power supply. The stimulator is a dual channel which can be programmed by a Palm PDA via infrared link and powered by rechargeable batteries (Fig. 4). [24]

\subsection{Medical modelling}

Computer modelling and simulation of human body and its behaviour are very useful tools in situations where it is either too risky to perform a surgical procedure or too costly for in vivo experiments. The modelling approach especially in biological

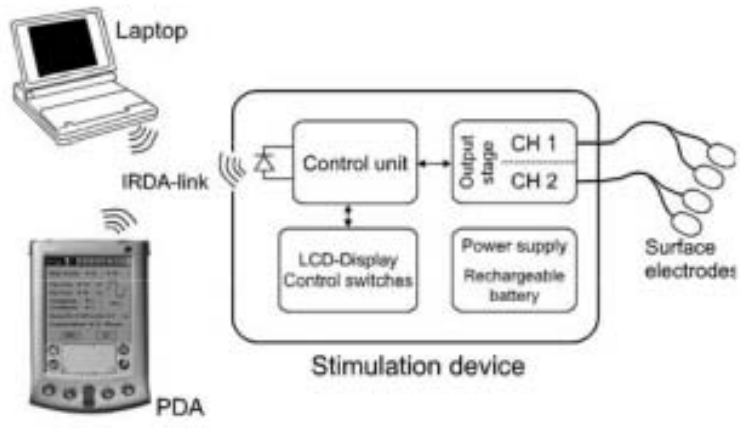

Fig 4 Schematic diagram of the surface electrode based 2-channel stimulator for denervated musculature [25]

system is designed according established criteria and objectives, indeed a complex model has such a high number of parameters that can not all be identified and accounted. Therefore the modelling work is usually constraint to describe accurately certain characteristics and approximating or ignoring others.

Medical modelling is generally applied to represent behaviours of biological systems under certain conditions: describing for example mechanical and/or electrical behaviour, visualizing in 3Dimension human anatomy and changes in geometry, representing pathologies or difficult geometry, simulate biological conditions and surgical treatments and study tissue proprieties or correlate different characteristics.

Another field of medical modelling is the so called biomodelling which is defined as the creation of highly accurate physical models of human anatomy directly from medical scan data. The process involves capturing human anatomy data, processing the data to isolate individual tissue or organs and eventually use rapid prototyping techniques to provide the physical model. Rapid prototyping is the general name used to describe computer-controlled machines that are able to manufacture physical items directly from 3Dimensional computer data. Figure 1.6B and $\mathrm{C}$ show the outcomes from two different rapid prototyping techniques: 3D printing* and stereolithography** respectively.

The main stages of medical modelling process are:

- Medical imaging

o Select the optimal modality such as CT or MRI

o Set the appropriate protocol which enable a proper visualization of the region of interest

o Scan the patient

- Export the data from the scanner in an appropriate format

- Process the data: segmentation of the organs or tissue to be modelled 


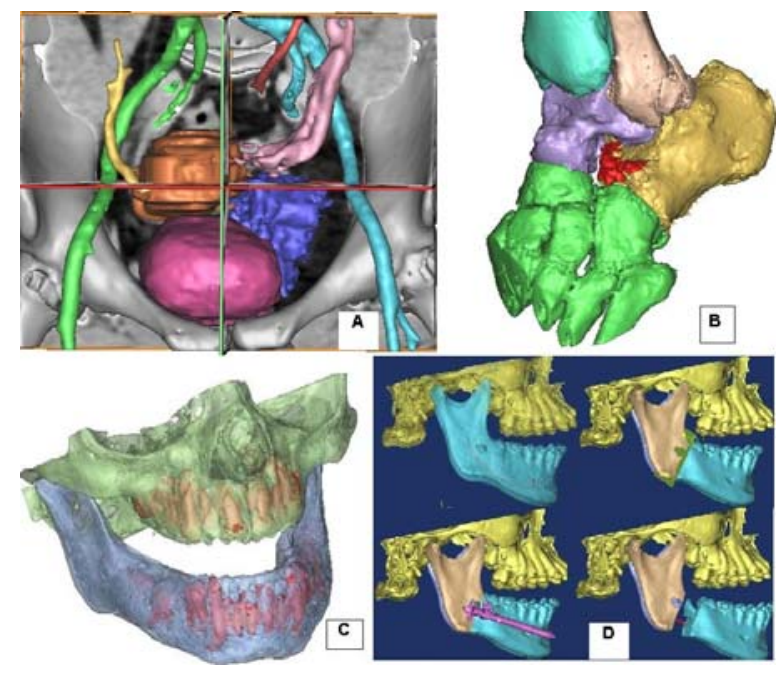

Fig 5 Different clinical applications of medical modelling in Iceland: (A) diagnostic support, (B) surgery planning, (C) challenging medical case, (D) surgery simulation.

- Further processing to analyse and model other biological characteristics and proprieties (such as tissue composition, fibre activation, bone mineral density etc.) or rapid prototyping to produce a physical model

*3D Printing: A three dimensional object is created by layering and connecting successive cross sections of material (powder compound)

**Stereolithography: Fabrication process utilizing a vat of liquid UV-curable photopolymer resin and UV laser to build parts a layer at time

\subsubsection{Medical Modelling in Iceland}

In Iceland since 2003 3Dimensional modelling techniques have been important research topics and an exciting development project. The main propose is integrating modelling from medical images in the health practise.

3Dimensional modelling (Fig. 5) is currently used in key clinical activities such as:

- Diagnostic, supporting pathologist with visualization of difficult anatomical case

- Planning surgery in complicate orthopaedic and maxilla facial operation

- Patient follow up in craniofacial trauma, studying structural changes in bone and soft tissue

- Patient compliance providing computer simulating in maxilla lengthening

Beside the clinical applications just mentioned, the modelling work in Iceland was especially successful in the planning treatment of intraoral distraction for mandibular lengthening becoming a validated service. This surgical procedure it is used for reconstruction of skeletal deformities. It involves gradual, controlled

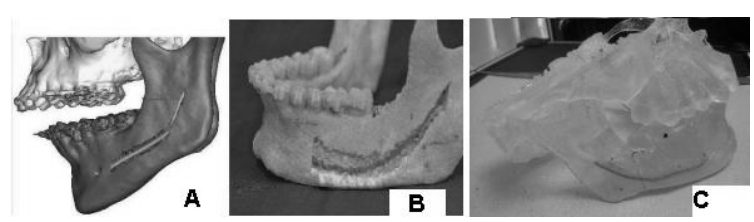

Fig 6 (A) Computer model of a jaw where the mandible nerve is rendered visible using segmentation tools. (B) $3 D$ print out of jaw (C) Stereolithographic model

displacement of surgically created fractures on both side of the jaw which results in simultaneous expansion of soft tissue and bone volume.

The distraction device is mounted either side of the jaw (Fig. 1.5D). Adjusting the distraction device and positioning it is frustrating for surgeon and takes time during surgery. Beside the surgeon must take very good care to not damage the mandible nerve during the surgery. For these reasons CT scan images are used to create 3Dimensional models of the jaw and segmentation technique are applied to render visible the mandible nerve (Fig. 1.6A). The computer model is then prototyped using 3Dimensional printing (Fig. 1.6B) or stereolithography technology (Fig. 1.6C). The physical model is used for planning previous to the start of the operation start and the distraction devices are adapted on the model "bending process" and then serialized prior the application on the jaw. This preparation allows considerable time saving in the operation theatre (Fig. 6).

Over 20 surgery planning of intraoral distraction for mandibular lengthening in three years demonstrate a diminishing of the operation time between $25 \%$ up to $35 \%$ (1 - 1,5 hours).

\subsubsection{Modelling FES}

Although in recent year's technology and methods have tremendously improved allowing more selective stimulation to target muscle membrane, better materials for the electrodes and better knowledge of the biological systems, the validation and the optimization of some FES application remain still an important issue. In the stimulation of paraplegic FES technology current objectives are:

- Improve selectivity in muscle fiber stimulation o Development of implantable device

- Reduce risk of injury during application since patients don't feel pain.

o New materials and geometry for electrodes

o Better control and monitoring of FES-parameters during treatment.

The use of models which can describe and quantify biological phonemes and interactions is very important to reproduce physiological conditions and experiment setting which can not be tested clinically. For this 


\section{D Modelling and monitoring of denervated muscle under FES}

European Journal Translational Myology - Basic Applied Myology 2011; 21: 31-94

reason the role of computer modelling and simulation is more and more important in research and development.

As base of all FES functioning principles there is the quantitative expression of the active membrane behaviour described by Hodgkin and Huxley [26] after experiments with squid axons. Many variants and modifications of their initial model exist [27].

In the frame of the RISE project the first computer model of FES of denervated human thighs was developed in 1999 by Reichel [28]. Further development of Reichel work was made in 2003 [29] by Martinek which developed a method to determine activated regions and by Mandl in 2005 which calculate the effects of muscular anisotropy during FES using segmentation techniques [30].

In recent years new computational tools such as FEMLAB [31] were used to model membrane physiology and FES parameters. Particularly interesting are the studies on the activation patterns in the stimulated human thigh; stimulation parameters and electrodes parameters have been optimised in relation to the muscle fiber activation [32]. Validation of these models and results was made using T2parameter in MRI measurement. The aim is to estimate in vivo activated regions in human skeletal muscle [33].

\subsection{Aim of the study}

Image processing techniques and 3Dimensional modelling are applied to display and study behaviour in muscle and bone from SCI patients with complete paralysis in the lower limbs and long term denervated muscle undergoing FES treatment.

The PhD work was born and developed in the frame of the European project RISE. The data processed and developed during the study are gathered from three Icelandic patients voluntary participating to the project. The study aims are:

- Development techniques to monitor muscle growth and model geometrical changes

- Study structural changes and tissue composition in degenerated muscle and bone

- Correlate induced muscle contraction and bone mineral density changes

\section{DATA, PROCESSING AND PATIENTS}

\subsection{Data acquisition}

During the RISE project to monitor morphological changes in muscle, x-ray computed tomography (CT) scans are taken at different time during the FES treatment. The method employed in Vienna consists of taking CT scans at $10 \mathrm{~cm}$ intervals from the trochanter major to the knee [20]. A comparison of two scans, taken at different times but at the same level on thigh shows the muscle growth in that specific place during the time period between the two scans. Comparing these scans yields an estimation of the total muscle growth and changes in fat and bone tissue. However, this method can give a qualitative indication and some quantitative information of how the muscle is changing but does not show the growth of the whole muscle. Beside the data from 5 - 10 scans are not enough to make a three-dimensional reconstruction of muscle and bone and therefore geometrical changes in these tissues aren't measured.

In Iceland the medical imaging methodology employed to monitor the muscle growth is different; volumetric information of the thigh are required for 3-dimensional visualization and modelling. Therefore Icelandic RISE patients are scanned with a spiral-helical CT scanner which allow fast and dose optimised scans.

\subsubsection{Spiral- Helical CT}

Computed tomography (CT) utilizes an x-ray tube, which rotates axially or helically around the patient, and a diametrically opposed array of detectors, which detect the residual radiation traversing the body [34].

Spiral or Helical CT differs from normal CT scanners because the gantry rotates continuously during scanning and the data acquisition is combined with continuous movement of the patient through the gantry. The path of the X-rays can be described as a spiral or helix, hence the name helical or spiral CT. These scanners can acquire large volume very quickly. This is advantageous for a number or reasons:

- Patient can hold their breath for the entire study therefore reducing motion artefact and radiation dose

- Due quick scanning time it allows more optimal use of intravenous contrast enhancement

- The study is quicker than the equivalent conventional CT allowing for higher resolution in the same study time.

The data obtained from spiral $\mathrm{CT}$ is perfect for $3 \mathrm{D}$ imaging. CT data are digitized and converted to crosssectional images with the help of powerful array processors. These devices record, digitize, store, and tomographically reconstruct hundreds of projection images acquired at many angles about the patient, using mathematical algorithms. Generation of a thin, fan-shaped, collimated x-ray beam and concurrent thin collimation of the detectors are required in order to reject scatter radiation emanating from outside the interrogated section. High efficiency, low-noise detectors with a linear response over a wide dynamic range and with high signal-to-noise ratio measurements are necessary. The scanner device used is called LightSpeed Pro 16 and is produced from General Electric Health Systems (Fig. 7).

The matrix detector in this scanner gives optimal 3D imaging, providing high resolution images (down to $0.625 \mathrm{~mm}$ slice thickness) and large coverage. It is built on GE patented HiLight poly cristalline ceramic technology that provides $99 \%$ absorption efficiency. The HiLight matrix detector ensures high performance 


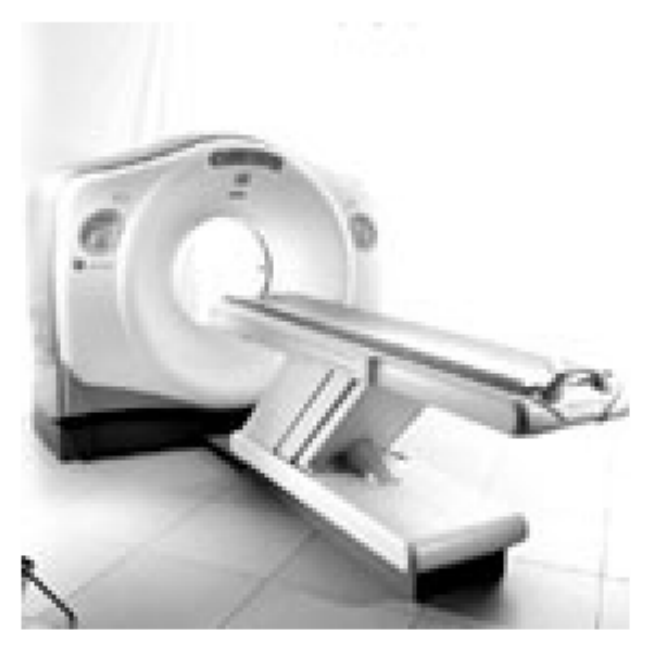

Fig 7 LightSpeed Pro 16 scanner used at Landspitali. Produced from General Electric Health Systems.

response in low signal conditions such as those encountered using low dose protocols in highly attenuating patient regions. LightSpeed Pro16 does have a dose optimization program, thus the patient gets the smallest radiation possible each time [35].

\subsubsection{Image representation}

Every acquired CT slice is subdivided into a matrix of different size, from a minimum of $128 \times 128$ up to 1024 $\times 1024$ volume elements (voxels). Each voxel is traversed, during the scan, by numerous X-ray photons and the intensity of the transmitted radiation measured by detectors. The image is then calculated from this array of voxels converting it into a matrix of picture elements (pixels) with each pixel assigned the attenuation value of the corresponding voxel. From these intensity readings, the density or attenuation value of the tissue at each point in the slice can be calculated. Specific attenuation values are assigned to each individual voxel. The degree of attenuation depends on the energy spectrum of the X-rays as well as on the average atomic number of the mass density of the patient tissue. The transmitted intensity is given by equation 2.1 .

$$
I_{t}=I_{0} e^{-\mu \Delta x} \quad \text { (eq. 2.1) }
$$

Where I0 and It are the incident and transmitted beam intensities respectively, $\Delta \mathrm{x}$ is the length of the x-ray path through the material and $\mu$ is the $\mathrm{x}$-ray linear attenuation coefficient, which varies with tissue type.

The x-ray tube rotates axially or helically around the patient therefore the whole effect of this motion is the gathering of millions of accurate absorption measurements through the body. These are taken at all angles through the slice, thus providing us with an enormous amount of information about the composition of the slice. The readings from the detectors are fed into a computer which derives the absorption of the material in the path of the $x$ ray beam, equation 2.2 .

$$
\text { Absorption }=\log \frac{x-r a y \_ \text {Intensity }}{\text { Detector_reading }}
$$

Where the absorption factor is the same is the same quantity indicated with $\mu$ in eq. 2.1 .

Image reconstruction is the process of generating an image from the data, which consists of the measurements of the detected x-ray transmission intensities. The CT reconstruction process results in a two-dimensional matrix of floating numbers in the computer which range from 0.0 up to values equal to 1.0. These numbers correspond to the average linear attenuation coefficient of the tissue contained in each voxel.

Most computer display hardware requires integer numbers and therefore the linear attenuation coefficients are rescaled to an integer range that encompasses 4096 values, between -1000 and 3095 . This scale is called CT number or Hounsfield unit (HU) and it is expressed by the following formula:

$$
C T \text { Number }=1000 \cdot \frac{\mu_{\text {pixel }}-\mu_{\text {water }}}{\mu_{\text {water }}}
$$

given pixel ( $\mu$ pixel) is equal to that of water, the CT number will be 0 . If $\mu$ pixel is less than $\mu$ water the CT number will be negative and negative CT numbers are typical for air spaces, lung tissues and fatty tissues.

Values of $\mu$ pixel greater than $\mu$ water will results in positive CT numbers. Dense tissue such as bone has large positive number. In figure 8 a thigh cross section is segmented according to the main composing tissues and corresponding HU values are indicated.

\subsubsection{Use of CT numbers for tissue characterisation}

The use of absolute CT numbers for in vivo tissue characterization and comparison must take into account a number of technical and geometrical factors. Indeed correlation between CT Hounsfield unit from one CT investigation to another and from one CT scanner device to another can be very difficult, especially when dealing with small changes in the tissue substance.

Indeed, there is a wide range of CT numbers observed for a given tissue type as a result of scanner performance, and if absolute CT numbers are used for diagnosis then these machine-related variations must be less than the differences thought to be significant. Studies performed on different CT scanner measuring CT number from phantoms shows significant differences in absolute CT numbers, even between scanners of the same manufacturer and model. CT 


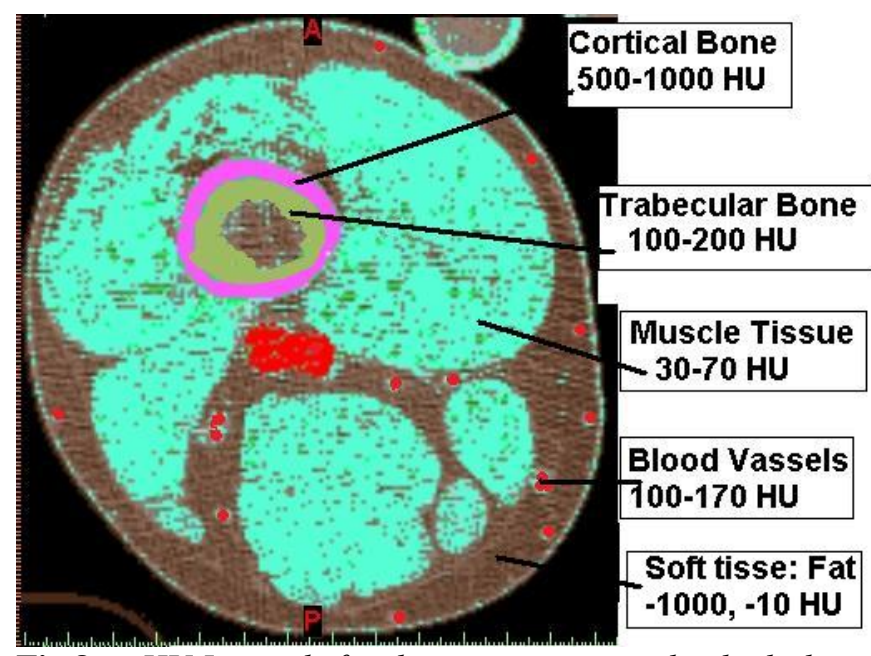

Fig 8 HU Intervals for the main tissues in the thigh: bone, muscle, blood and fat

numbers differs also between single phantom scan, depending from the location in the scan, and the format of this variability change from one scanner to another. [36, 37, 38]

Various physical factors can influence the CT number representation during a scan session. The parameter that mostly affects the accuracy and the spatial distribution of $\mathrm{HU}$ values is the applied voltage across an X-ray tube; this amplitude is measured in kilo volt $(\mathrm{kV})$ and determines the highest X-ray quantum energy and therefore the attenuation coefficient. CT number distribution is also influenced by phantom (or patient) orientation and position in scan aperture. Therefore it is necessary to know and account these variability's when CT numbers are used for tissue characterization and comparison $[39,40]$.

In order to account these facts and allow a reliable comparison between data set, the scanning protocol was established in the beginning of the clinical trial and some calibration tools used during every measurement since 2005. As can be seen in the next paragraph the scanning parameters aren't all exactly the same but varying into a range which depend from the different scanned volume (the patients have different length in their lower limbs) and from an ongoing imaging quality assessment (when a change in the protocol demonstrate to improve imaging then it is implemented).

\subsubsection{Acquisition parameters and scanning protocol}

A spiral CT is taken every four months on the Icelandic subgroup of three patients included in the RISE study. Within more than four-year of treatment each patient was scanned twelve times (first CT scan in December 2003, last in September 2008) creating a tremendous amount of data. The scanned region starts above the head of the femur and continues down to the knee joint, with both legs covered by one scan. Each study results in a total of 750-1000 CT slices, depending on the patient's size. These information allow accurate reconstruction of the 3Dimensional volume from the scanned region. The CT slides are square arrays containing $512 \times 512$ pixels, each pixel representing 4096 possible shades of gray, meaning that it is represented with a 12-bit value. The voxel has two dimensions equal to the pixel and the third dimension represents the slice thickness of the CT scan. Therefore a typical data set have a size between $512 \times 512 \times 750 \times 12=2.4$ GBit and $512 \times 512 \times 1000$ $\times 12=3.1$ GBit.

The main acquisition parameters are as following listed and described in the following sections.

\section{Slice Thickness}

The slice thickness $(\mathrm{mm})$ is $1.25 \mathrm{~mm}$. This value defines the third dimension of the volume element (voxel). The other two dimensions height and width are defined by the field of view.

\section{Pitch}

The scans are taken with a pitch of $0,625-0.8 \mathrm{~mm}$. In the helical scan the pitch is defined as the ratio between the table travel per rotation $(\mathrm{mm} / \mathrm{rot})$ and the $\mathrm{X}$-ray beam width $(\mathrm{mm})$. The helical pitch range is 0,5 - $2 \mathrm{~mm}$. Having a fixed x-ray beam: to higher pitch correspond a higher distance covered by the table during a gantry rotation.

$$
\text { pitch }=\frac{\text { table_travel }}{(\text { xray_width }) \times(\text { gantry_rot })}=N \in[0,625-0,8]
$$

This parameter set the distance between slices and since this distance is minor compared with the slice thickness which is set on $1,25 \mathrm{~mm}$, then there will be an overlapping of reconstructed slices which is suppose to increase the quality of the 3D image (Lee, 2002)

\section{Field of View (FOV)}

The field of view (mm) varies between 17 and $19 \mathrm{~cm}$ depending from the scanned volume. It is the physical distance over which the image is taken. Usual values are $10-50 \mathrm{~cm}$. If the scanning region is big then also the distance from where the image is taken must be big (high FOV). Beside, since the reconstruction matrix is $512 \times 512$ pixels then to bigger field of view correspond also a bigger pixel size. Since the FOV isn't fixed exactly but varying between 17 and $19 \mathrm{~cm}$ also pixel size slightly changes from one study to another. In volume and density measurement these differences in pixel size must be taken into account to allow correct comparison between data. 


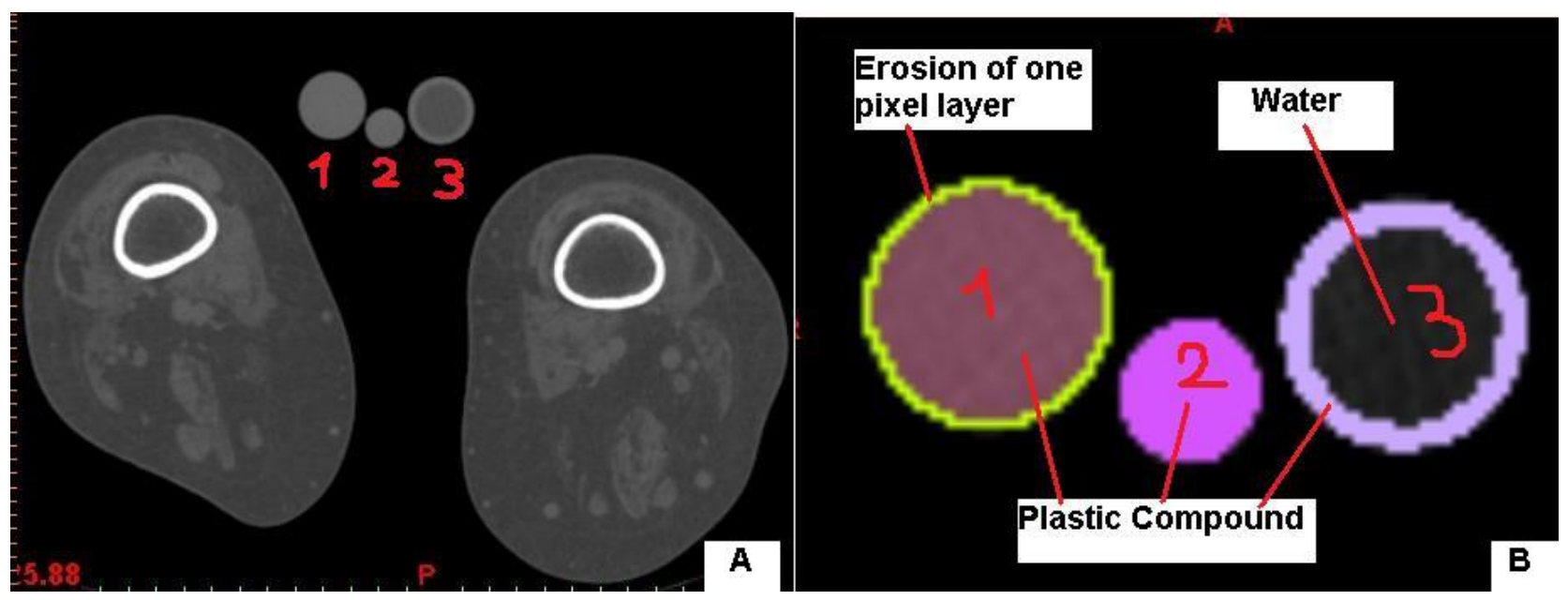

Fig 9 (A) Cross section showing the position of the calibration tools with the patient thighs. (B) Zoom on the calibration tools: $n r .1$ and 2 have different diameters (1,5 and 2,5 cm respectively) but are made of plastic compound. Nr.3 is hollow and filled with water. In the nr.1 it is visible the erosion of one pixel layer to avoid nose on the edges.

\section{Tube potential}

The tube potential is the voltage between anode and cathode, with higher potential electrons accelerate more and have higher energy. Usual values are 80$140 \mathrm{kV}$, in our scanning protocol the tube potential is set on $120 \mathrm{Kv}$.

\section{Volume radiographic exposure}

The volume radiographic exposure $(\mathrm{Cvol})$ is measure of the average radiographic exposure over the total volume scanned, where $\mathrm{C}$ is the radiographic exposure (mAs).

$$
C_{\text {vol }}=\frac{C}{\text { CT pitch factor }}=N \in[250-400]
$$

(mAs/mm)

(eq. 2.5)

In our scanning protocol the volume radiographic exposure is between $250-400 \mathrm{mAs}$.

\subsubsection{Calibration}

The CT number is in general not reliable indicator for biological tissue if it is used as absolute value as discussed in paragraph 2.14. Indeed even in the same material and during the same scan we can expect a certain range of variability for the $\mathrm{CT}$ number. The evaluation of this range is very important for accurate measurement of density and volume. To estimate these variability, since 2005 the patient scanning is performed using some calibration tools.

The calibration tools are three long cylinders made with different materials which are scanned with the patients; they are positioned on the patient bed on top or between the legs, in parallel with the patient thighs (figure 9, A). One cylinder is hollow and filled with water (indicated with nr.3 in Fig.9, B) while the other two are full filled with same plastic compound but having different diameters (nr.1 of 2,5 cm and $\mathrm{nr} .2$ of $1,5 \mathrm{~cm}$, Fig.9, B).

The cylinders are supposed to be used as reference for the HU measurements since the water and plastic compound have stable and homogenous composition. Theoretically $\mathrm{HU}$ values should not change inside the water (assuming the value $0 \mathrm{HU}$ ) and varying minimally within the plastic compound and along the tubes. Anyway the hollow cylinder showed not to be very reliable as calibration tool because of the air bubble generated inside the water which makes the HU distribution along the tube to assume values below zero and oscillate too widely. Instead the plastic tube with the larger diameter is used to calibrate the measurement and to estimate the range of tolerance where HU numbers can characterise correctly tissue composition inside the thighs. The larger diameter tube is preferred to the smaller one because it allows a better cross sectional analysis. Indeed to avoid dust or other material and partial volume effect to change local absorption on the tube edge, one pixel layer is removed from the surface as showed in the tube nr.1 in figure 9, B.

Using the calibration tool the HU mean values and standard deviation are calculated on whole cylinder volume and in five different cross sections along it. This kind of measurement is though to measure the HU shift depending from the scanned volume. In fact the tube is scanned parallel to the thighs and the five cross sections are taken starting from the patella level and ending at trochanter level with inter distance between cross sections of $10 \mathrm{~cm}$ (Figure 10). The tissue volume scanned is a lot different at patella level compared to 


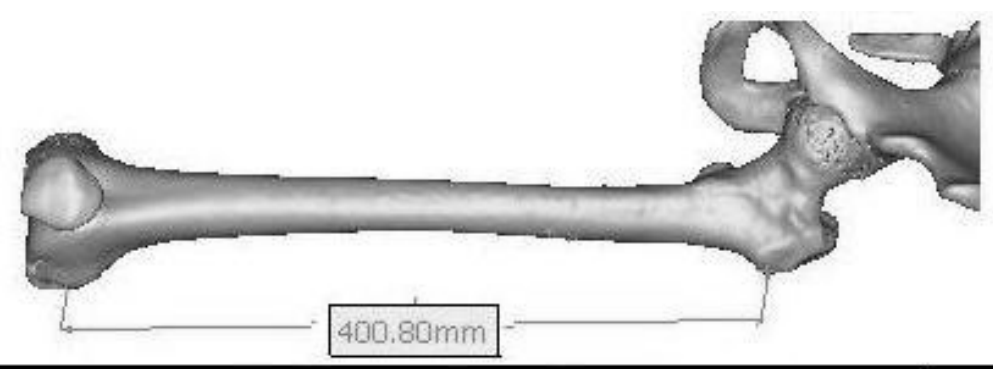

\begin{tabular}{|c|c|c|c|c|c|c|}
\hline \multirow{2}{*}{$\begin{array}{l}\text { Data-set } \\
\text { (year) }\end{array}$} & \multicolumn{5}{|c|}{$\begin{array}{c}\text { Position: patella- trochanter } \\
\text { Mean- SD Standard Deviation (HU) }\end{array}$} & \multirow{2}{*}{$\begin{array}{c}\text { Total } \\
\text { Mean- SD } \\
\text { (HU) }\end{array}$} \\
\hline & $0 \mathrm{~cm}$ & $10 \mathrm{~cm}$ & $20 \mathrm{~cm}$ & $30 \mathrm{~cm}$ & $40 \mathrm{~cm}$ & \\
\hline 2005 & 95,8 - 56,8 & $97,8-38,0$ & $97,4-40,3$ & $96,8-46,1$ & 95,2 - 58,9 & $110,8-7,4$ \\
\hline 2006 & $107,7-23,7$ & $105,0-21,0$ & 103,5 - 20,6 & $104,4-21,0$ & $101,1-21,9$ & $109,0-7,6$ \\
\hline 2007 & $97,0-24,4$ & $94,3-26,2$ & $94,7-29,2$ & $101,7-25,3$ & $100,0-30,2$ & $110,9-6,7$ \\
\hline 2008 & $100,4-28,0$ & $100,1-24,4$ & $94,6-26,2$ & $98,0-27,2$ & $93,1-28,3$ & $106,7-9,6$ \\
\hline
\end{tabular}

Fig 10 The above picture shows the 3D model from the femur bone. The region, patella-trochanter, calibrated with the plastic tubes is displayed. In the table the mean $H U$ values and the standard deviations are measured for the whole calibration tool (last column) and in 5 cross sections between patella and trochanter. The distance between cross sections is $10 \mathrm{~cm}, 0 \mathrm{~cm}$ is assumed the position at patella level.

the one at trochanter level cross. Therefore these measurements enable the quantification of the CT number deviation due the different volume crossed by the $\mathrm{x}$-ray beam.

The figures in Fig. 10 show two important facts which will be taken into account in the measurements session: - The mean HU value for the whole plastic tube doesn't change remarkably between studies, indeed the maximum difference is 4,1 HU (Mean values difference in the last column in figure 10).

- In the same study along the plastic tube the variability between cross sections mean density values are higher (Up to $11,1 \mathrm{HU}$ at $0 \mathrm{~cm}$ ). These differences are depending on the dimension of the scanned cross sectional area of the tissue.

Therefore the HU intervals that will be created and used to discriminate and measure different tissues will have to account for both the HU displacement between different studies and in the same study between different cross sectional areas.

\subsection{Image processing tools}

The CT scan data are processed, compared and developed using different computational tools. Very powerful image processing and segmentation tool has been used to isolate and make 3-Dimensional reconstruction of bone and muscle tissue. The software name is MIMICS and it is developed by a company called Materialise with head quarter in Leuven Belgium. Mimics is an image processing software package for 3-dimensional design and modeling, it generates and modifies surface 3D models from stacked medical images such as Computed Tomography (CT), Confocal Microscopy, Micro CT, and Magnetic Resonance Imaging (MRI) through image segmentation done in the STL form.

STL file describes a raw unstructured triangulated surface by the unit normal and vertices of the triangles using a three-dimensional Cartesian coordinate system. To monitor the denervated muscle changes a number of method were developed and used. All these methods are based on image processing techniques and segmentation techniques, for this reason a brief list of definition and main concept are as described in the following sections.

\subsubsection{Segmentation techniques}

In the analysis of the objects in images it is essential that we can distinguish between the objects of interest and "the rest." This latter group is also referred to as the background. The techniques that are used to find the objects of interest are usually referred to as segmentation techniques: segmenting the foreground from background. Any segmentation have as a result a set of pixels which are related to each other because of the applied segmentation role, this set of pixel is usually called segmentation mask or only mask.

In computer vision, segmentation refers to the process of partitioning a digital image into multiple regions (Sets of pixels), the results of segmenting an image is 


\section{D Modelling and monitoring of denervated muscle under FES}

European Journal Translational Myology - Basic Applied Myology 2011; 21: 31-94

to produce a binary mask image which fulfil the segmentation criteria.

Several general-purpose algorithms and techniques have been developed for image segmentation. Since there is no general solution to the image segmentation problem, these techniques often have to be combined with other knowledge in order to effectively solve the problem.

For example the main segmentation problem faced in this work was to separate muscle bellies from surrounding tissues when the surrounding were muscles as well, and therefore having the same HU values. Indeed in this case there are not automatic tools available to separate such regions and a combination of different segmentation tools must be used and adapted.

\section{Thresholding}

This segmentation technique is based upon a simple concept.

A parameter $\Theta$ called the brightness threshold is chosen and applied to the image $\mathrm{a}[\mathrm{m}, \mathrm{n}]$ as follows:

If $a[m, n] \geq \theta$ then $a[m, n]=$ object $=1$

Else $a[m, n]=$ background $=0$

In principle, the test condition could be based upon some other property than simple brightness. In our application each pixels display an HU value which varies in a range from -1024 to 3072 depending from the tissue. If the interest is to visualize tissues in a range HU1-HU2 then the test condition will appear as follow:

If $H U 1 \geq a[m, n] \geq H U 2$ then $a[m, n]=$ object $=H U m n$ Else $a[m, n]=$ background

Example, thresholding figure 11: $\mathrm{HU} 1=1, \mathrm{HU} 2=5$, object $=$ red, background $=$ white

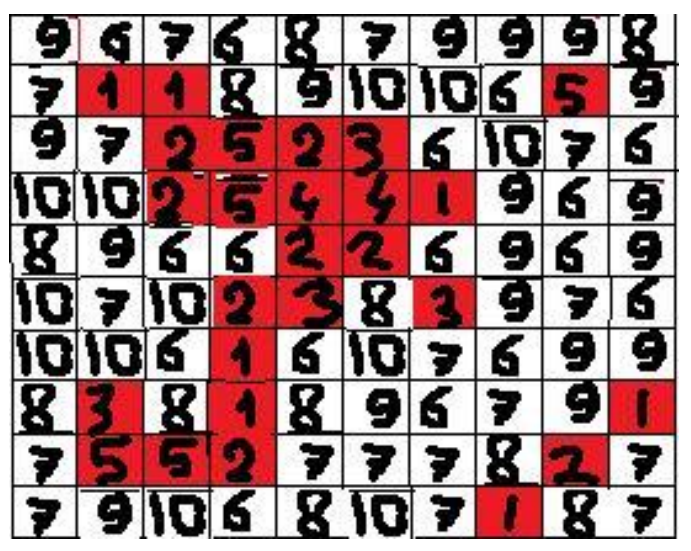

Fig 11 Matrix $10 \times 10$, the pixels displayed are with $H U$ values from 1 to 10. The threshold is [1,5], and all pixels in this interval are selected (red coloured)

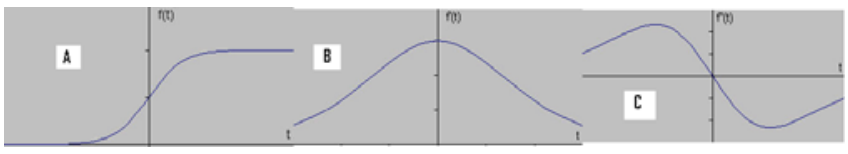

Fig 12 (A) function $f$; (B) first derivate of function $f$; (C) second derivate of function $f$.

\section{Edge Detection}

Edge detection is a terminology in image processing and computer vision, particularly in the areas of feature detection and feature extraction, to refer to algorithms which aim at identifying points in a digital image at which the image brightness changes sharply or more formally has discontinuities.

There are many ways to perform edge detection. However, the most may be grouped into two categories, gradient and Laplacian. The gradient method detects the edges by looking for the maximum and minimum in the first derivative of the image (Fig. 12, A-B). The Laplacian method searches for zero crossings in the second derivative of the image to find edges (Fig. 12, C).

A pixel location is declared an edge location if the value of the gradient exceeds some threshold. Since edges will have higher pixel intensity values in the first derivative than those surrounding it, once a threshold is set a comparison between gradient value and threshold detect the edge whenever the threshold is exceeded (Fig. 13, A). Furthermore, when the first derivative is at a maximum, the second derivative is zero. As a result, another alternative to finding the location of an edge is to locate the zeros in the second derivative (Fig.13, B).

In this work the edges are characterised by the boundary between different tissues: muscle-bone, muscle-fat and muscle-muscle. In the first two cases the differences are very evident and edge detection is rather easy, in the latter case the connective tissue separating different muscle bellies is often too thin to perform a reliable separation and therefore other tools must be used.

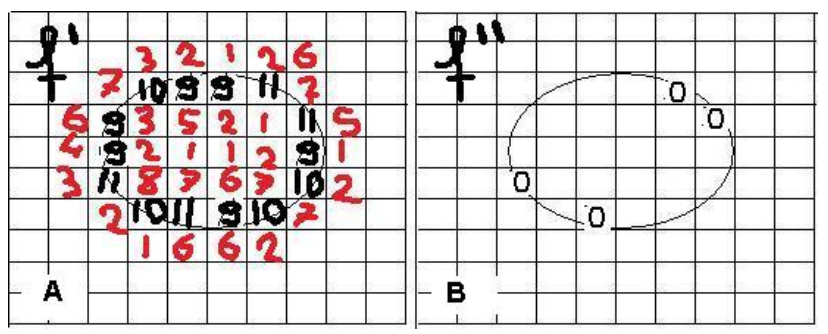

Fig 13 Edge detector technique. Implementation of gradient method (A) and Laplacian method (B) 


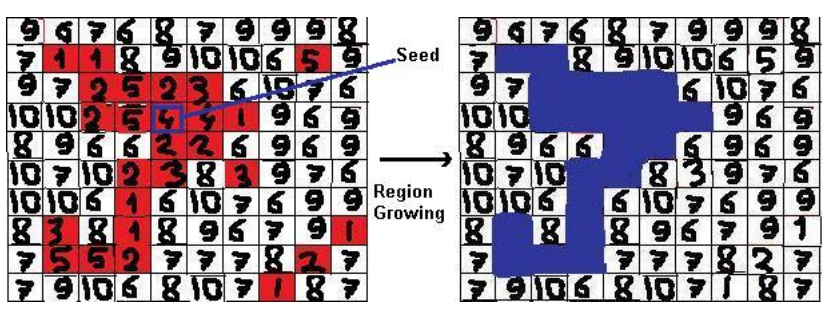

Fig 14 Implementation of region growing method using as seed the pixel framed with blue

\section{Region Growing}

Region Growing is an approach to image segmentation in which neighbouring pixels are examined and added to a region class if no edges are detected. This process is iterated for each boundary pixel in the region. An initial set of small areas are iteratively merged according to similarity constraints. Start by choosing an arbitrary seed pixel and compare it with neighbouring pixels. The region is grown from the seed pixel by adding in neighbouring pixels that are similar, increasing the size of the region. This whole process is continued until all pixels belong to some region. In our application region growing is applied on muscle regions where edges are clearly defined and the surrounding pixels are merged to the original seed only when their $\mathrm{HU}$ values belong to a certain interval [HU1, HU2].

\section{If $H U 1 \geq a[m, n] \geq H U 2$ then $a[m, n] \epsilon$ pixel seed}

Else $a[m, n]=$ background

Example, region growing figure 2.8: $\mathrm{HU} 1=1, \mathrm{HU} 2=$ 5 , seed =blue initial pixel seed 4 , background $=$ white

\section{Morphology Operations}

Morphology Operations perform actions on the 'form' of a mask. The morphological functions take or add pixels from a source mask copying the results in the target mask

The main morphology operations are "dilate and erode “(Fig. 15):

- Dilate: dilate will add a pixels layer to the edges, in figure 2.9 the additional layer is coloured with green.

- Erode: remove a pixels layer from the edges, the operation erode followed with a region grow allow separating parts (Fig. 16)

Morphological operations are used in the muscle segmentation to improve the quality of the model for instance separating noisy pixels from muscle and bone edges.

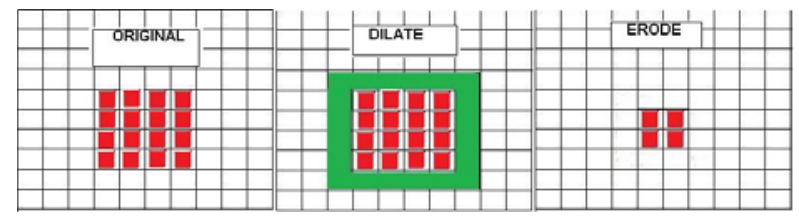

Fig 15 Morphological operation: Dilate and Erode of denervated muscle under FES

- Basic Applied Myology 2011; 21: 31-94

Boolean operations

The Boolean operations allow different kinds of combinations based on two masks: union, subtraction and intersection. The specific pixel intensity (HU in our case) isn't changed by Boolean operations. The change operates by Boolean function is in the threshold limits of the resulting mask as described in the following:

- $\quad$ Subtraction (Minus) A-B: Threshold value = Threshold value mask A

- Intersection $\mathrm{A} \cap \mathrm{B}$ : lower threshold $=\max$ (low threshold mask A, low threshold mask B)), higher threshold $=$ min (high threshold mask $\mathrm{A}$, high threshold mask B))

- Union A B : lower threshold $=$ min (low threshold mask A, low threshold mask B)), higher threshold $=\max$ (high threshold mask $\mathrm{A}$, high threshold mask B))

In muscle segmentation the Boolean operation are used for example to quantify for instance the amount of fat and connective tissues present in the muscle volume:

- Mask A: representing a muscle belly volume (which has been already segmented) including all the characterising tissues such as fat, muscle, connective tissue and water

- $\quad$ Mask B: representing the fat contained in all data set (Mask B is created simply applying a threshold operation which select pixels within the proper $\mathrm{HU}$ value displaying fat)

- $\quad \mathrm{A} \cap \mathrm{B}=$ Mask $\mathrm{C}$, the result of the intersection will represent the content of fat into the segmented muscle belly volume.

\section{Editing tools}

Editing tools are usually used to complete the segmentation process; they consist of manual editing functions performed on the mask. They are used for instance to separate the mask from the surrounding when automatic tools aren't able to do it and/or to include certain regions that can not be acquired in automatic ways.

Editing operations are drawing, erasing, or restoring the image with a certain threshold value. A typical application of the editing tools in this work was to separate the border muscle-muscle.

\subsection{Patients and compliance}

The patients participating to the FES trial in Iceland

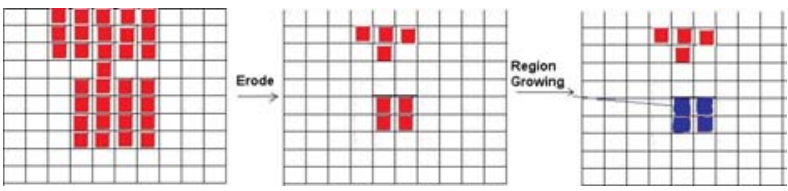

Fig 16 Use of erode to separate two masks 
3D Modelling and monitoring of denervated muscle under FES

European Journal Translational Myology - Basic Applied Myology 2011; 21: 31-94

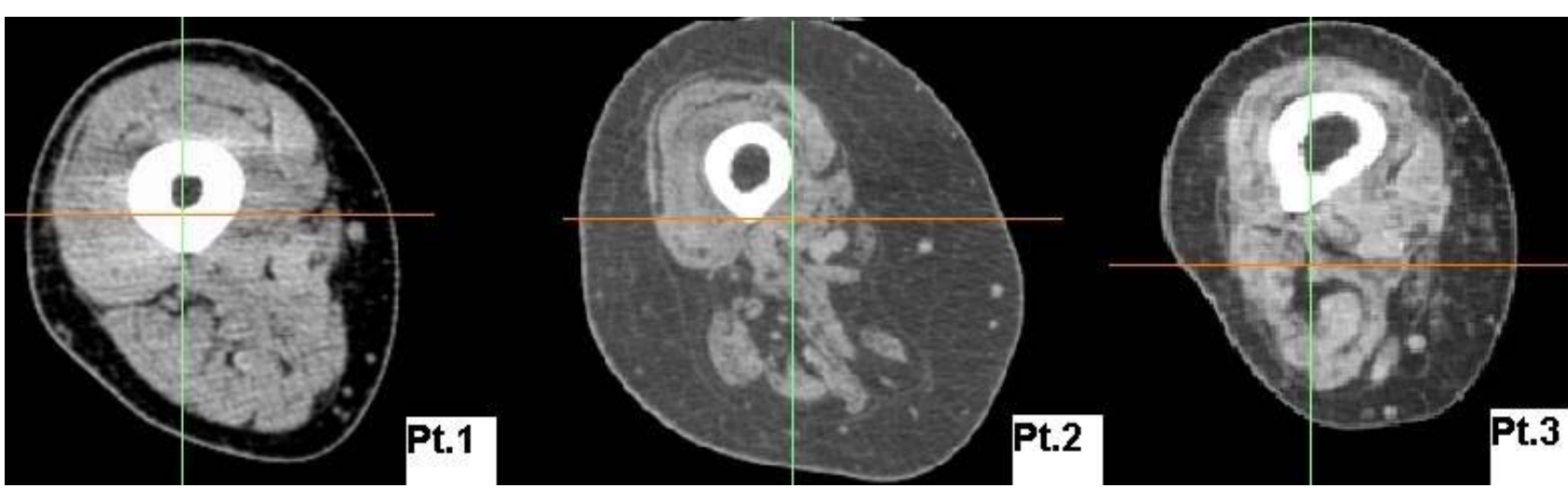

Fig 17 Initial right thigh cross sections from the three Icelandic patients

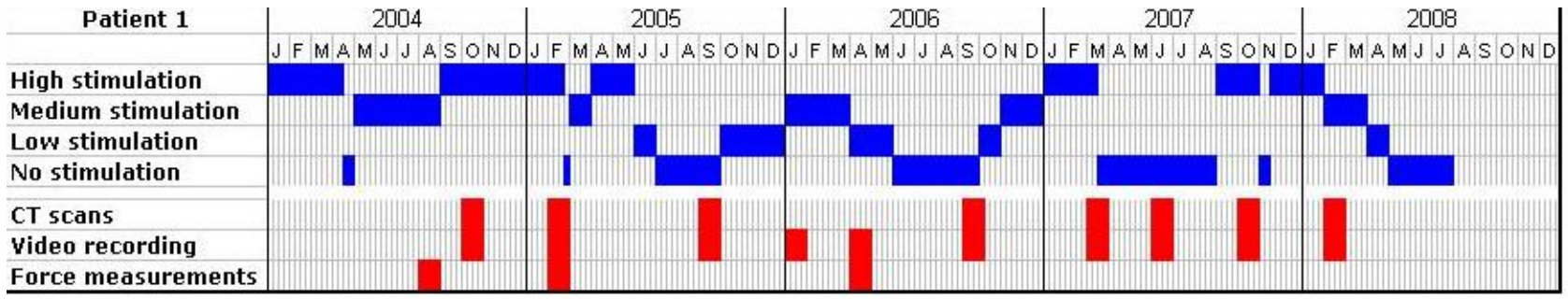

Fig 18 Compliance chart for patient 1

\begin{tabular}{|l|l|l|}
\hline Time & Clinical remarks & Recovery time \\
\hline Nov. 2004 & Pressure sores at the sacrum & 2 months \\
\hline Feb - Mar 2005 & Pressure sores at the sacrum & 1 months \\
\hline Oct - Dec 2005 & Pressure sores at the sacrum & 2 months \\
\hline Jul - Sep 2005 & $\begin{array}{l}\text { Fracture of ankle + stomack problems weight } \\
\text { loss }\end{array}$ & 3 month \\
\hline April 2006 & Operate to the cholecystitis & 1 month \\
\hline June 2006 & Sepsis admitted to hospital weight loss=9 kg & 1 month \\
\hline Nov 2007 & Shoulder operation & 1 month \\
\hline
\end{tabular}

Table 1 Remarkable clinical occurrences for patient 1 which interrupted the FES treatment

are three. They are paraplegic with lower extremities fully denervated but starting the FES treatment with different levels of muscles degeneration (Fig. 17).

\section{Patient nr. 1}

He is born in 1973 and got injured in a car accident in 2003. He fractured at ThXII and has complete L1 lesion of lower motor neurone (LMN) type. The patient joined the RISE project 10 months after the injury and therefore still had little degeneration in the muscle (Figure 17, first picture) and great potential to achieve standing quickly. Unfortunately he has not been always therapy compliant and beside this, several clinical problems has occurred during these years
(Table 1). In figure 18 the patient compliance is displayed since the FES treatment began: from January 2004 to June 2008. Three regimes of stimulation are defined according to the number of stimulation sessions per week:

- High stimulation, 5-6 times a week

- Medium stimulation, 3-4 times a week

- Low simulation, 1-2 times a week

In figure 18 are also seen the measurement session: CT scans, video recording and force measurement.

Patient nr. 2

He is born in 1979 and got injured when he fell of a roof in 1999. The lesion is ThXI-ThXII of LMN type 


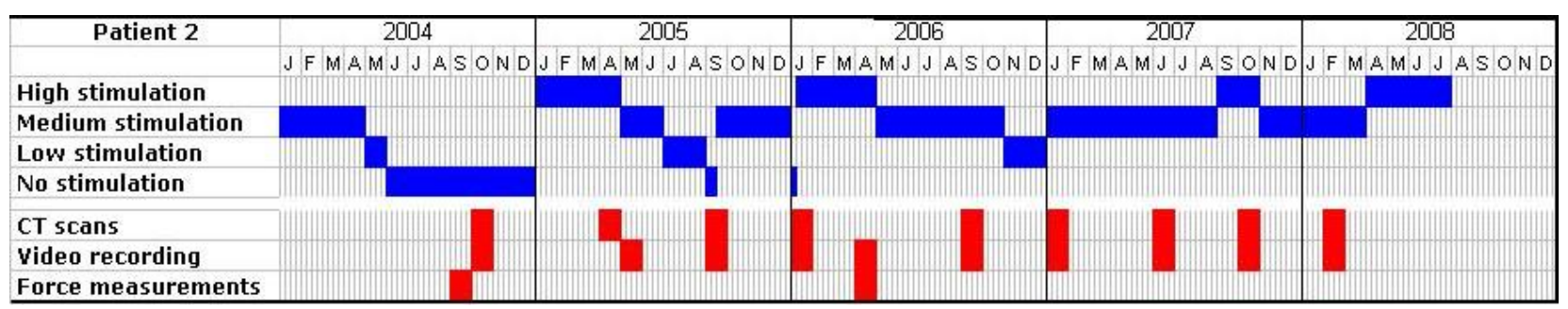

Fig 19 Compliance chart for patient 2

\begin{tabular}{|l|ll|}
\hline Time & Clinical remarks & Recovery time \\
\hline $\begin{array}{l}\text { Summer } \\
2004\end{array}$ & \multicolumn{3}{|l|}{ Colitis ulcerosa } & 2 months \\
\hline Oct-Des 2004 & $\begin{array}{l}\text { Many burns sores after gel 3 months } \\
\text { stim }\end{array}$ \\
\hline
\end{tabular}

Table 2 Remarkable clinical occurrences for patient 2 which interrupted the FES treatment

with luxation. He has complete flaccid ThXI syndrome with paralysis and areflexia in legs and medium atrophy. The patient joined the RISE project 4 years after the injury therefore his muscles are long term degenerated. The patient has been rather compliant as seen from figure 19; though in 2004 due some clinical problems (Table 2) stimulation was interrupted for long period. Additionally the efficiency of the FES treatment has been negatively influenced by the abundant adipose tissue surrounding the quadriceps muscles as can be seen from the middle picture in figure 17.

\section{Patient nr. 3}

He is born in 1949 and got injured in a car accident in 1996. The lesion is from ThXII-LII of LMN type with luxation at LI-LII and complete ThX syndrome. He has some hyperalgesia at ThX-XI level. The patient joined the RISE project 7 years after the injury therefore with muscle severely degenerated muscle (Figure 17, picture on the right). The patient has in general been compliant (Figure 20) except in the periods where clinical problems have occurred (Table 3 ).

\section{PUBLICATIONS}

3.1 Morphological changes in rectus femoris muscle: advanced image processing technique and 3 -dimensional visualization to monitor denervated and degenerated muscles treated with functional electrical stimulation

This work was published in Basic Applied Myology 17 (3\&4): 133-136, 2007
3.1.1 Abstract
This study demonstrates qualitatively and quantitatively how functional electrical stimulation (FES) restores volume and morphology of denervated degenerated muscle. For this purpose a novel technique was developed to isolate and monitor muscle growth. During

3 years of electrical stimulation, complete 3dimensional reconstructions of rectus femoris muscles from patients with long-term flaccid paraplegia were made in regular interval of four months. Spiral computer tomography and advanced image processing tools were developed and used to monitor and measure selected muscle regions influenced by the stimulation. The complete morphology of rectus femoris muscle (RFM) was reconstructed at different therapy stages.

The results show remarkable changes of shapes and volume: 3-dimensional imaging of rectus femoris muscle demonstrates the restoration process during the therapy.

\subsubsection{Introduction}

Experimental and clinical research provides strong evidence of FES being a powerful tool for regeneration, functional restoration and maintenance of denervated musculature. The European project called RISE (QLG5-CT-2001-02191) proposes a novel clinical rehabilitation method for patients with permanent and non-recoverable muscle denervation in their legs $[20,25]$. The main goal of the RISE project is to use electrical stimulation to restore standing in paraplegics with long-term DDM. 


\begin{tabular}{|c|c|c|c|c|c|c|}
\hline Patient 3 & 2004 & 2005 & 2006 & 2007 & 2008 & \\
\hline & $J F M A M J J A S O N D$ & $J F M A M J J A S O N D$ & $J F M A M J J A S O N D$ & $J F M A M J J A S O N D$ & $J F M A M J J$ & ASOND \\
\hline High stimulation & & & & & & \\
\hline Medium stimulation & & & & & & \\
\hline Low stimulation & & & & & & \\
\hline No stimulation & & & & & & \\
\hline CT scans & & & & & & \\
\hline Video recording & & & & & & \\
\hline Force measurements & & & & & & \\
\hline
\end{tabular}

Fig 20 Compliance chart for patient 3

\begin{tabular}{|lll|}
\hline Time & Clinical remarks & Recovery time \\
\hline Jan-mars 04 & Pressure sore at the gluteus & 3 months \\
\hline Oct 2004 & $\begin{array}{l}\text { Widespread bone infection } \\
\text { osteosynthesis }\end{array}$ & 3 months \\
June 2005 & Fracture of right prox tibia & 1 month \\
April 2007 & Embolism in leg & 1 month \\
Nov 2007 - Feb 2008 & Burns soars on leg & 4 months \\
\hline
\end{tabular}

Table 3 Remarkable clinical occurrences for patient 3 which interrupted the FES treatment

There being no possibility of re-innervation, patients with long term denervated degenerated muscles were refused FES-training for years. It was also widely believed that FES was unsuitable in DDMtraining. Kern, however, not only showed that DDM patients can indeed be trained with FES using surfaceelectrodes, but also that FES training is able to restore the ability of muscles to perform tetanic contractions, that, in fact, are essential to prevent/recover atrophy by loading the muscles $[23$, 41, 42, 43].

The crucial indicator for the therapy is, of course, muscle growth. The effects of the treatment were monitored, e.g., by morphological and histochemical analysis of muscle biopsies as well as by clinical neurological, neurophysiological mechanical, and radiological methods [25]. To track changes in the size and shape of the quadriceps muscle, computer tomography (CT) scans were taken at $10 \mathrm{~cm}$ intervals from the trochanter major to the knee. A comparison of two scans taken at the same level at different times shows the muscle growth in that specific place during the period between the two scans. Comparing five scans, taken at $10 \mathrm{~cm}$ intervals, yields an estimate of the total muscle growth and changes in fat and bone tissue. It does not, however, show the growth of the whole muscle, as data from only 5 scans are not sufficient to make a three-dimensional model of muscle, bone, or other tissue. Additionally, denervated musculature loses its normal morphological shape over time, making both localization and identification of the different muscle tissue difficult. This paper describes a method to isolate the RFM from the other muscles of the thigh and to measure volume and density during 3 years of electrical stimulation. Through an accurate 3-dimensional reconstruction, the morphological changes are visualized and monitored.

\subsubsection{Material and Methods}

Spiral CT

Every four months a spiral CT was taken of the RISE Study's Icelandic subgroup of three patients with a complete spinal cord injury of flaccid type. The spiral CT scan starts above the head of the femur and continues down to the knee joint, both legs being covered by one scan. They are taken with a pitch of approximately $0.625 \mathrm{~mm}$, resulting in a total of about 750-900 CT slices, depending on the patient's size. Each slice has $512 \times 512$ pixels, and each pixel has a grey value in the Hounsfield scale of 4096 grey scale values, meaning that it is represented with a 12-bit value. A total data set from a single scan is therefore $512 \times 512 \times 750 \times 12=2.36$ GBit. This data set gives a complete threedimensional description of the tissue, including the muscles and bones in both upper legs [42].

Area of Interest

The CT scan data were imported into a special image processing and editing computer program called MIMICS [44]. These files are rather big, considerably limiting the computer processing capacity; they were, therefore, preliminarily divided in two: one file for the right leg and another for the left. The area on the thigh where the quadriceps muscle is stimulated is of particular interest. The goal was to select the muscle bellies with the highest dose of 


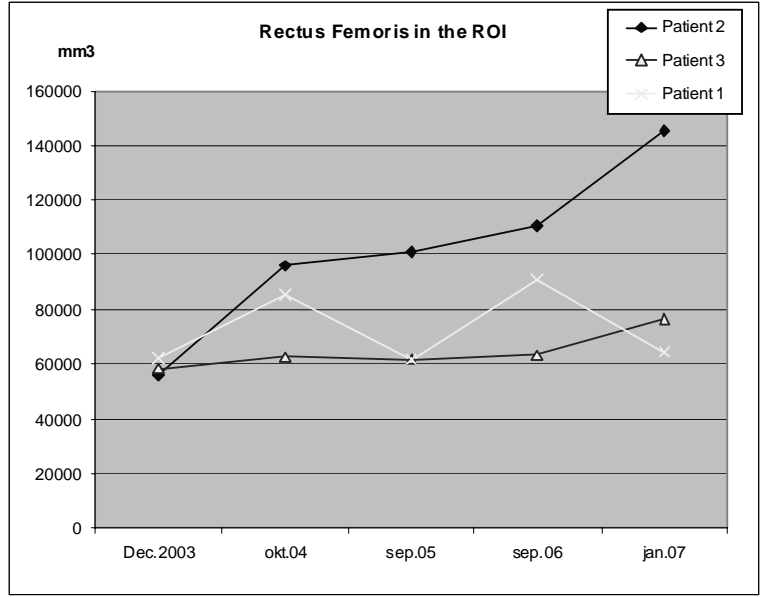

Fig 21 Changes of rectus femoris volume during FES therapy.

electrical stimulation and monitor their changes during therapy. Another reason is the importance of developing a methodology allowing duplication of the same area at different times. According to the RISE protocol large surface electrodes were applied on the quadriceps muscle. Because of muscle morphology, the rectus femoris is the most exposed part of the quadriceps to direct stimulation. The RFM was therefore chosen as the region of interest and monitored during the therapy.

Muscle segmentation

Objects scanned by CT are coded according to the Hounsfield scale, a quantitative scale for describing radio density where different values are ascribed to air, water, fat, bone and muscle. Image processing tools are used to segment the images and differentiate the diverse objects represented on the CT slide. The first step in image segmentation is thresholding: individual pixels with certain $\mathrm{HU}$ value are marked if their value is included in the customized $\mathrm{HU}$ interval representing muscles. The HU interval chosen in this application is -25 to 129 which guarantees the visualization of the muscles tissue in the different degrees of degeneration. The next step is to isolate the RFM from its surrounding muscles. The main difficulty in the segmentation process is the continuity, in term of HU values, between the RFM and the adjacent muscle tissue making conventional image processing tools useless for this purpose. The muscle was therefore segmented manually, directly on the CT slide, using advanced editing tools and the following method: the trans axial slide that visualizes RFM close to the patella is selected. A contour is drawn manually around the muscle; all pixels inside the curve are marked. The shape created on the first slide is projected on to the next. The muscle border on the new slide is slightly different, and the shape will

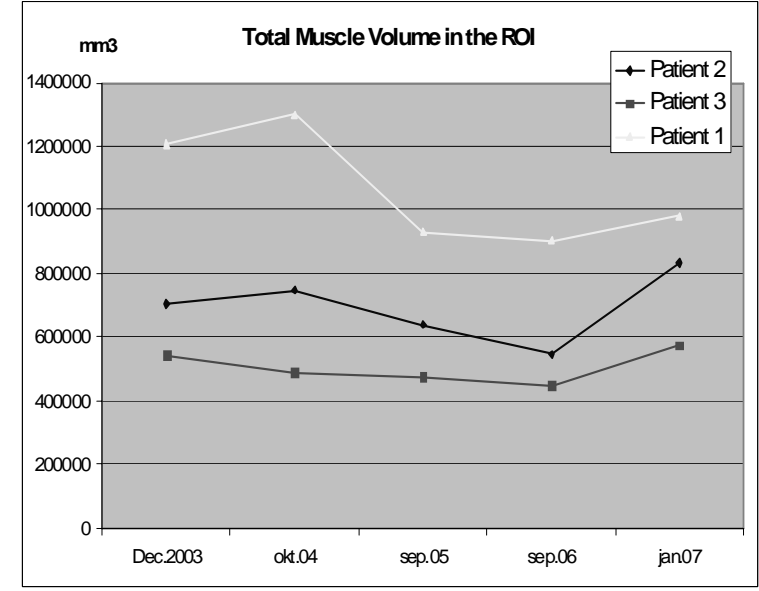

Fig 22 Changes of total volume of thigh muscles during FES therapy.

therefore be adapted to fit the new muscle contour. Again all pixels inside the curve are marked and the shape projected forward. This process continues until the muscle ends on the pelvis. Finally the RFM is completely isolated from its surroundings. A mask containing the selected pixels is created. This mask represents the RFM and includes information on muscle density and volume. Using special software tools the rectus femoris mask can be converted into a $3 \mathrm{D}$ object allowing monitoring of shapes and morphologies. Finally, using standard image processing tools, femur and patella bones are also reconstructed in 3-D. In this way RFM can be represented correctly and its changes during the FES therapy easily localized and understood.

Measurements

In this work the RFM from one Icelandic patient (named Patient 2) was isolated and monitored according to the RISE stimulation protocol from December 2003 to January 2007. The patient has been denervated since July 1999. Some measurements were also performed on the two other Icelandic RISE patients (Patient 1 has been denervated since February 2003 and Patient 3 from January 1996). Both patients began the stimulation program at the end of 2003. For these measurements a Region of Interest (ROI) is defined: $140 \mathrm{~mm}$ below the head of the femur and $100 \mathrm{~mm}$ above the knee joint.

The rectus femoris is part of the quadriceps muscle with the highest stimulation exposure; changes in this muscle are an index to validate the RISE therapy. The following data are presented:

- Monitor changes in shape and morphology: 3Dimensional reconstruction of rectus femoris at different times during the therapy (Figure 23).

\subsubsection{Results}




\section{D Modelling and monitoring of denervated muscle under FES}

European Journal Translational Myology - Basic Applied Myology 2011; 21: 31-94

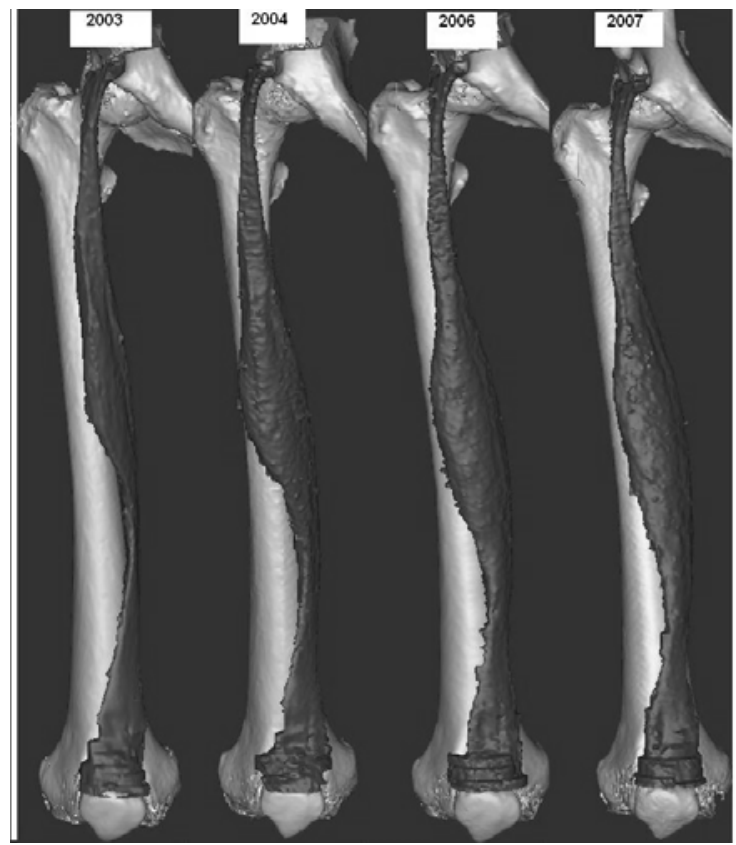

Fig 23 Total muscle volume changes during FES. The 3-dimensional reconstruction of RFM at different times during the therapy show remarkable improvement, clearly demonstrating the restoration process of normal morphological shape.

The data in Figure 23 show volume growth of Rectus Femoris among the three Icelandic RISE patients. The growth rate is very different between patients:

- RFM in Patient 1 has an oscillating trend due to long interruption of treatment during the FES therapy. December 2003 - January 2007, RFM growth: $+4 \%$

- RFM in Patient 2 shows the best growth rate. Especially in the years 2004 and 2006, patient was more compliant, and then the volume growth was better. December 2003 - January 2007, RFM growth: $+84 \%$

- RFM in Patient 3 has a very slow, but continue growth rate due to long denervation time before onset of therapy. December 2003 - January 2007, RFM growth: $+20 \%$.

The data in Figure 3.2 show the total volume growth in the thigh, among the three Icelandic RISE patients.

- Patient 1: December 2003 - January 2007, Volume in the thigh: $-16 \%$

- Patient 2: December 2003 - January 2007, Volume in the thigh: $+20 \%$

- Patient 3: December 2003 - January 2007, Volume in the thigh: $+8 \%$

Figure 3.3 shows the results from the 3dimensional reconstruction of RFM at different times during the therapy. Remarkable improvements are naked-eye recognized, clearly demonstrating the restoration process toward a normal shape.

\subsubsection{Conclusion}

FES stops muscle degeneration and promotes muscle growth in the regions where it is applied. The new technique implements segmentation of muscle bellies and isolation of RFM improving the monitoring of the RISE therapy. Compared with the old method where muscle changes were only estimated on a few trans axial slides at fixed positions on the thigh, the new monitoring technique allows measures of volume and density on the whole muscle belly. Additionally, the combination of volume data with 3-dimensional imaging of the muscle provides qualitative and quantitative information on the otherwise hidden muscle behaviour. In this paper data from the monitoring of the rectus femoris of one Icelandic RISE patient (patient 2) is published. Four data sets were developed with the new monitoring technique: at the beginning of the project and approximately once a year during the therapy period. The results clearly demonstrate muscle growth and restoration of normal muscle shape and morphology. In three years of stimulation, rectus femoris has almost doubled its volume.

Though the stimulation was applied to the whole quadriceps muscle, it seems that the RFM is the only muscle truly gaining from the therapy. An explanation could be that the thicker fat layer surrounding the other quadriceps muscles prevents the stimulation from being as effective.

The next step, therefore, would be to implement this methodology: measure other patients and segment and measure all the muscles in the thigh. The muscle growth rate is of course influenced by patient compliance and by possible clinical problems affecting the patients during therapy. For instance, having to stop the stimulation protocol for a few weeks can lead to the loss of a muscle mass previously gained over several months. It is crucial to correlate and measure muscle changes in order to understand how denervated musculature grows and to measure changes in volume and density at different regimes of stimulation. Segmentation of muscles can be a highly useful tool to perform such investigations. Information from RFM segmentation contributes to the adjustment and improvement of the stimulation protocol, for instance in changing electrode position or stimulation patterns. Furthermore this information on muscle behaviour is an important basis for new development such as: stimulator, electrodes and implantable devices. Finally the segmentation process and monitoring methodology developed in this work is an accurate tool to validate the RISE therapy and provide a better understanding of the behaviour of denervated muscle. 


\section{D Modelling and monitoring of denervated muscle under FES}

European Journal Translational Myology - Basic Applied Myology 2011; 21: 31-94

\subsection{Restoration of muscle volume and shape Induced by electrical stimulation of denervated degenerated muscles: qualitative and quantitative measurement of changes in rectus femoris using computer tomography and image segmentation}

This work was published in Artificial Organs, Vol. 32, No. 8, 2008

\subsubsection{Abstract}

This study demonstrates in a novel way how volume and shape are restored to denervated degenerated muscles due to a special pattern of electrical stimulation. To this purpose, Spiral Computer Tomography (CT) and special image processing tools were used to develop a method to isolate the rectus femoris from other muscle bellies in the thigh and monitor growth and morphology changes very accurately. During 4 years of electrical stimulation, three-dimensional (3D reconstructions of the rectus femoris muscles from patients with long-term flaccid paraplegia were made at different points in time. The growth of the muscle and its changes through the time period are seen in the 3D representation and are measured quantitatively. Furthermore, changes in shape are compared with respect to healthy muscles in order to estimate the degree of restoration. The results clearly show a slow but continuing muscle growth induced by electrical stimulation; the increase of volume is accompanied by the return of a quasi-normal muscle shape. This technique allows a unique way of monitoring which provides qualitative and quantitative information on the denervated degenerated muscle behaviour otherwise hidden.

\subsubsection{Introduction}

In the frame of the EU-funded RISE project, patients with lower motor neuron lesions and denervated and degenerated muscles were treated with electrical stimulation, with the aim of restoring muscle mass and force. It has been shown to be possible to build up mass, force, and function of long-term denervated and degenerated muscles with the use of electrical stimulation [22]. Moreover, the developed muscle force should enable the legs to bear the patient's weight, allowing him to stand up and maintain the standing posture, for example, between parallel bars, with the aid of electrical stimulation. In order to reach and maintain these results, muscles are stimulated for up to $2 \mathrm{~h}$ per day, 6 days a week. This has to be done without interruption for an extended period of time. As part of the RISE project in Iceland, three paraplegic patients with fully denervated and, to a great extent, degenerated muscles in the lower extremities are treated with electrical stimulation. These patients with long-term flaccid paraplegia have no hope of regaining their muscle function. Traditional treatment only aims at side effects of the injury. Moreover, in comparison with patients with a spastic paraplegia, they often suffer more from several severe complications, for example, decubitus ulcers, reduced bone density with a high risk of fractures, severe muscle atrophy with decreased circulation, lower metabolism, etc. [46]. In conjunction with the clinical work, different monitoring methodologies are developed and applied to measure the therapy effects in quantitative and qualitative ways. The treatment effects on the patients are monitored, for example, by morphological and histochemical analysis of muscle biopsies as well as by clinical neurological, neurophysiological mechanical, and radiological methods [20]. To follow changes in size of the quadriceps muscle, computer tomography (CT) scans are taken at $10 \mathrm{~cm}$ intervals from the trochanter major to the knee. A comparison of two scans taken at two different times at the same position shows the muscle growth in that specific place during the time period between the two scans.

A comparison of five scans, taken at $10 \mathrm{~cm}$ intervals, yields an estimate of the total muscle growth and changes in fat and bone tissue. However, it does not show the growth of the whole muscle and its morphological changes and also is not enough to make a three-dimensional (3D) model of the muscle, bone, or other tissue.

In this article, a different approach to monitor muscle growth is described. A segmentation methodology is developed to selectively monitor muscle bellies. Changes of muscle shape, volume, and density are measured very accurately $[42,45]$.

This study demonstrates qualitatively and quantitatively how functional electrical stimulation (FES) restores volume and shape of denervated degenerated muscle.

\subsubsection{Material and Methods}

\section{Spiral CT scanning}

To enable monitoring of the whole stimulated muscles, spiral CT scans of the RISE Study's Icelandic subgroup are taken every 4-6 months. The scan starts above the head of the femur and continues down to the knee joint, both legs being covered by one scan. They are taken with a distance of $0.625 \mathrm{~mm}$ between slices, resulting in a total of about 750-900 CT slices, depending on the patient's size. Each slice has $512 \times$ 512 pixels, and each pixel has a gray value in the Hounsfield (HU) scale of 4096 gray-scale values, meaning that it is represented with a 12-bit value. A total data set from a single scan is therefore $512 \times 512$ $\times 750 \times 12=2.36$ GB. This data set gives a complete $3 \mathrm{D}$ description of the tissue, including the muscles and bones in both upper legs.

Threshold 


\section{D Modelling and monitoring of denervated muscle under FES}

European Journal Translational Myology - Basic Applied Myology 2011; 21: 31-94

In order to isolate the single muscle bellies and measure the growth, CT scan data are imported into a special image processing and editing computer program called MIMICS [44]. In this software environment, the $3 \mathrm{D}$ form of the muscle is reconstructed and certain regions of interest are extracted and isolated. The CT scan data gathered during the 4-year treatment are imported, organized, and segmented using MIMICS. To optimize computer memory and processing capacities, the left and right legs are processed separately.

To achieve the segmentation and then the isolation of the rectus femoris from the surroundings, the first step is to discriminate among the different tissues in the thigh using the HU scale. The HU scale is defined as a quantitative scale for describing X-ray attenuation properties of the tissue; different $\mathrm{HU}$ values are ascribed to air, water, fat, bone, and muscle. To select and visualize only the pixels representing muscle tissue, a threshold based on HU values is defined. A maximum and minimum value is established, and individual pixels are selected if their value falls in between the threshold values. Denervated muscles degenerate over time, muscle

fibers lose their normal arrangement and functionality; the whole muscle mass reduces dramatically, replaced with connective tissue and fat.

The interval chosen to visualize the denervated degenerated muscle tissue is $[-25,129] \mathrm{HU}$, while normal musculature has $\mathrm{HU}$ values between $40 \pm 20$ HU. The reason to use such a wide interval is to monitor the restoration process and the changes of muscle density, because during the stimulation therapy, muscle fiber is assumed to be regenerated, replacing the excessive connective tissue and fat.

\section{Segmentation process}

To allow selective and accurate monitoring of muscle changes, the cross-sectional area of the thigh is segmented into four areas, representing different muscle bellies or muscle groups. The segmentation process is not automatic but is based on the direct reorganization of the muscle bellies on the CT-scan slide. An initial cross section is selected from the data set. A contour is designed manually around the muscle belly cross section in order to isolate the selected

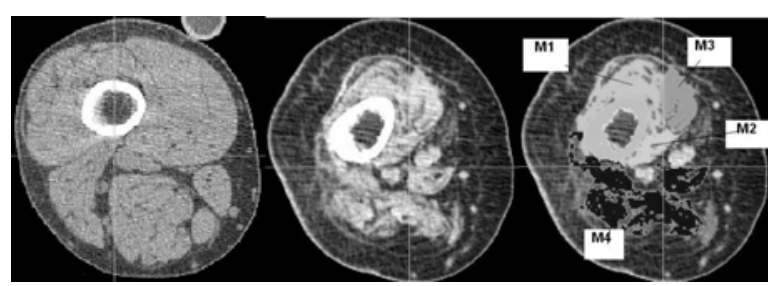

Fig 24 Left: healthy musculature. Center: denervated musculature. Right: coded masks of muscle bellies. region from the surrounding area. The shape created is projected to the next cross section and adjusted to fit the new muscle belly cross-sectional area. The process continues until all cross sections which build up the muscle are covered. In the segmentation process, the denervated degenerated musculature is divided into four regions: M1, M2, M3, and M4. M1 represents the vastus lateralis and intermedius, M2 the vastus medialis, M3 the rectus femoris, and finally, M4 the whole antagonist muscles (see Figure 24).

Rectus femoris volume measurements

Special attention was paid to segmenting of the rectus femoris muscle. There are two main reasons for the special interest in the segmentation and monitoring of the rectus femoris:

- The rectus femoris is closer to the electrodes during stimulation and therefore the most stimulated muscle.

- The rectus femoris musculature, because of morphology, can be segmented and isolated better from other muscles and tissue. Indeed, over time, denervated degenerated musculature loses mass and volume changing their shape, and therefore, many muscle bellies are no longer recognizable with respect to healthy muscles. The rectus femoris muscle instead remains roughly recognizable also when it is degenerated (Fig. 25).

Therefore, the rectus femoris is first isolated from other muscle bellies and then reconstructed in three dimensions. The data sets from different points of time are compared, and muscle growth is monitored very accurately. The process to isolate the rectus femoris and the following $3 \mathrm{D}$ representation of the muscle through time allow for the measuring and locating morphological changes induced by stimulation. The rectus femoris' evolution due to FES treatment is shown in figure 26.

\section{Quantitative shape measurements}

In order to quantify the changes in muscle shape, twodimensional figures of the muscle were examined (5). The figures show the frontal view of the muscle,

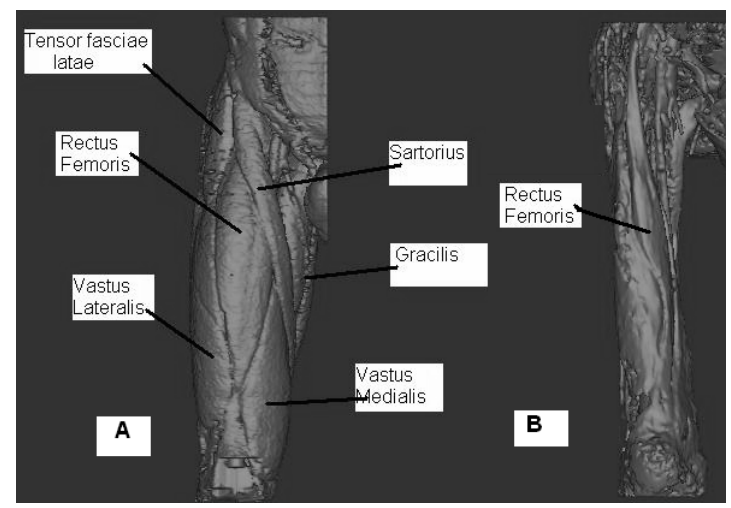

Fig 25 A: healthy thigh musculature. B: 3-years' denervated degenerated thigh musculature 


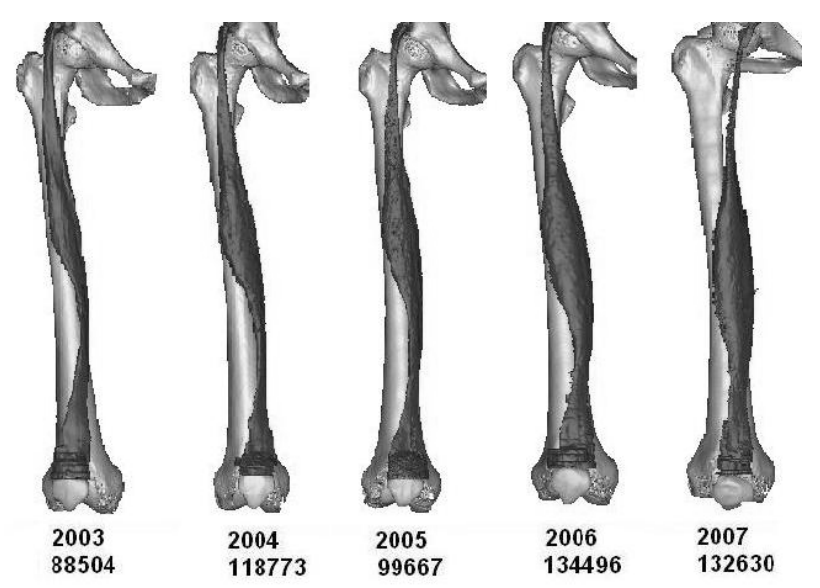

Fig 26 Rectus femoris $3 D$ reconstruction and volume (in cubic millimetres) at different points of time.

and the patella was used as an indication of the figure view being identical. Through morphological operations, the muscle edges were highlighted and used to determine a mean line throughout the length of the muscle. This mean line was then compared for the same patient at various stages in the therapy period and furthermore compared to data from a healthy subject. The comparison was done with root mean square error calculations of 100 samples along the length of the muscles:

$$
R M S E=\frac{1}{n} \sum_{1}^{n} \sqrt{\left(l(n)_{\text {healthy }}-l(n)_{\text {patient }}\right)^{2}}
$$

This is used to indicate a possibly diminishing error compared to a healthy subject, as the therapy progresses. Further calculations were made at the muscle region where the largest changes in shape were observed.

\subsubsection{Results}

The methodology developed in this work demonstrates itself as a powerful tool for muscle monitoring as well as allowing a better understanding of restoration mechanisms induced by electrical stimulation. Moreover, the results permit quantitative and qualitative measurement on the degenerate muscle otherwise hidden.

Developing a method to isolate muscle bellies from the surroundings and therefore measure it very accurately was deemed very important. Through these results, the RISE therapy is further validated, and correlation between muscle growths, density increase, and morphology changes were possible. The following are the quantitative results from a patient (labeled as Pt. 2) where the effect of the stimulation therapy is most apparent - a 29-year-old man, injured in 1999, started the stimulation program at the end of 2003. The muscle growth in the rectus femoris is

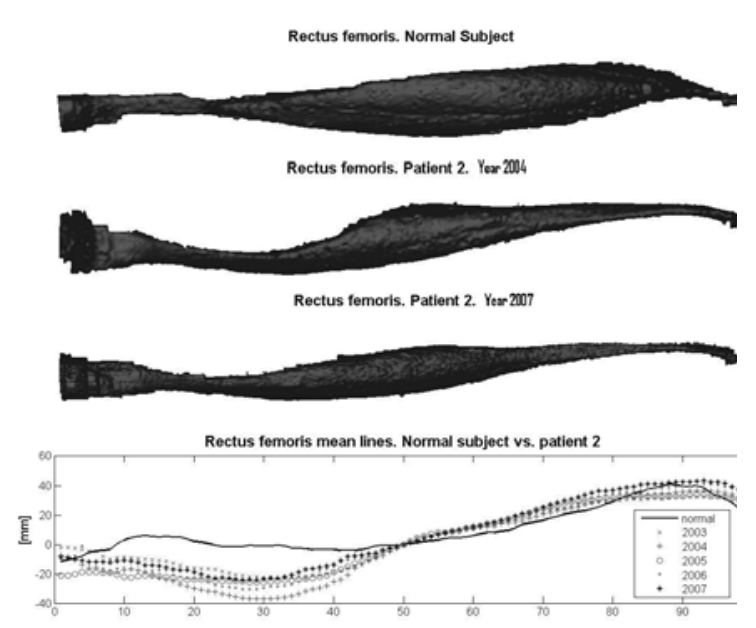

Fig 27 A comparison of the shape of the rectus femoris muscle as treatment progresses. Illustrated are pictures of the rectus femoris muscle of a normal subject as well as Pt. 2 at two different stages in the therapy. The bottom figure illustrates the comparison of the shape for normal subject (whole line) and Pt. 2 during the therapy progression (dashed lines). The largest changes in shape occur at $10-50 \%$ of the muscle length as indicated by

reasonable after 4 years of electrical stimulation with variable therapy compliance: from a volume of $88504 \mathrm{~mm} 3$ in December 2003 to a volume of $132630 \mathrm{~mm} 3$ measured in November 2007 (Fig. 26), a volume increase of $50 \%$.

Muscle density is also growing remarkably during the 4 years of stimulation, correlating to the volume results. Moreover, further measure of different sub volumes in the rectus femoris shows that density distribution in denervated muscles is different in comparison to healthy muscles. For instance, the lower part of the rectus femoris has significantly higher density compared to the values found on the whole muscle (Table 4). Besides the volume and density changes induced by electrical stimulation, the 3D reconstruction of the rectus femoris also shows certain changes in muscle shape which we have been localizing and quantifying.

The results from the quantitative estimation of morphological changes underline the fact that these changes are local and occur mostly in the lower part of the rectus femoris closer to the patella (Fig. 27). Along the medial and upper part of the muscle, changes of shape are negligible, although the volume growth is mostly in the central area of the rectus femoris. The difference in shape between the normal subject and the patient is largest in 2004, after just a short period of stimulation therapy, and thereafter the difference gradually decreases (Table 4, upper row). Comparison at the region of the most change (Fig. 27) 
3D Modelling and monitoring of denervated muscle under FES

Furnnean Inurnal Tranclational Mvolnov - Racic Annlied Mvoloov 2011 · 21 31_94

\begin{tabular}{|l|c|l|l|l|l|l|}
\hline Density [HU] & Healthy & 2003 & 2004 & 2005 & 2006 & 2007 \\
\hline Whole muscle & 66.2 & 37.7 & 36.2 & 40.9 & 42.1 & 41.7 \\
\hline 50\% of the length & 67.1 & 43.9 & 39.3 & 47.4 & 48.6 & 45.3 \\
\hline Volume (mm3) & 354158 & 88504 & 118773 & 99667 & 134496 & 132630 \\
\hline
\end{tabular}

Table 4 Mean density of the whole muscle (first row) and in the lower part (second row)

\begin{tabular}{|l|ll|ll|l|}
\hline RMS & 2003 & 2004 & 2005 & 2006 & 2007 \\
\hline Whole muscle & 9.2400 & 13.0200 & 11.9850 & 11.2167 & 10.1450 \\
\hline Selected area & 15.4268 & 25.0122 & 19.7683 & 19.2439 & 15.4146 \\
\hline
\end{tabular}

Table 5 The results from the root mean square error calculations. RMS-error was calculated both for the whole muscle length as well as for the area of the muscle where the most changes in shape occur (10-50\% of length) as indicated in Fig. 27

further emphasizes this trend, but with a clearer indication (Table 5, lower row).

\subsubsection{Discussion}

Clinical evidence shows that electrical stimulation cannot only stop muscle degeneration, but allows the muscles to grow again. The results of this work clearly demonstrate and quantify changes induced in muscle through the stimulation treatment. Further work should be done to study the correlation between the different restorations processes involved in muscle growth. For instance, important issues underlined in this work which deserve more attention are the density inhomogeneity and the changes of morphology characterizing long-term denervated muscle. A further indication of muscle regeneration is the muscle shape. The results of this study indicate that the changes in muscle shape occur primarily at the lower part of the rectus femoris muscle and that this change is gradually being reversed with the stimulation therapy. However, as the quantitative measures of shape changes in this study are performed only on the frontal plane of the rectus femoris, it is possible that further changes in other planes occur. In future work, this should be

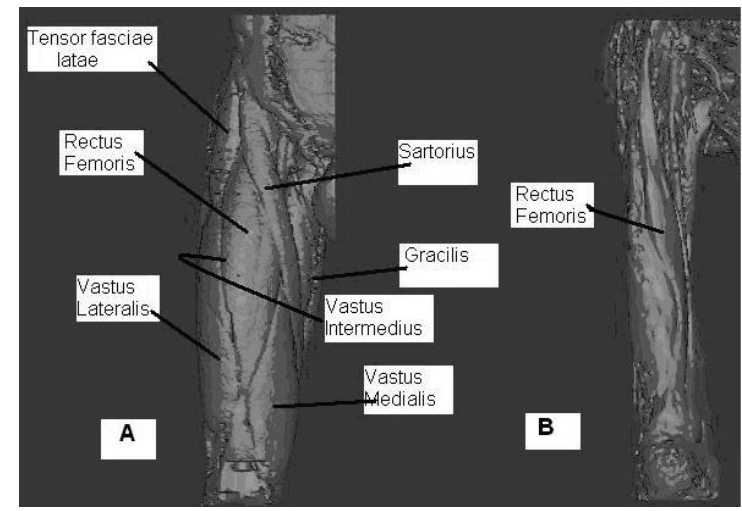

Fig 28 (A) Healthy thigh muscles. (B) 3-year denervated degenerate thigh musculature. addressed by multiplane studies and preferably a 3D study of the muscle shape.

Improved monitoring techniques and muscle modelling can also contribute to the development of new techniques and technologies to optimize stimulation protocols and electrode positions and, furthermore, be used as a base for new development designs such as stimulators, electrodes, and implantable devices.

Finally, the segmentation process and monitoring methodology developed in this work is not only an accurate method to validate the RISE therapy, but it is also a promising tool to improve understanding of denervated muscle.

\section{Modelling and Analysis of Rectus Femoris Muscle 4.1 Introduction}

The main target muscle for the electrical stimulation is the quadriceps femoris which includes four muscles on the front of the thigh: rectus femoris, vastus lateralis, vastus medialis and vastus intermedius (Fig. 28, A). The muscle volume in the quadriceps is very large and the muscles aren't uniformly activated by the electrical stimulation. In fact surface electrodes are by definition not selective and enormous energy is used to deliver electrical stimulus which can reach and cause contraction in the denervated muscles. Rectus femoris occupies the middle of the thigh and covers most of the other three quadriceps, it is closer to the surface electrodes and therefore more exposed to the stimulation as also demonstrated qualitatively in recent studies using T2 mapping [33]. Hence, it is of special interest to monitor and study the behaviour of rectus femoris, model certain characteristics and measure stimulation effects accurately.

In this chapter medical image, segmentation techniques and special computational tools are developed and used to isolate rectus femoris from other muscle bellies and analyse in novel way growth and muscle restoration. 


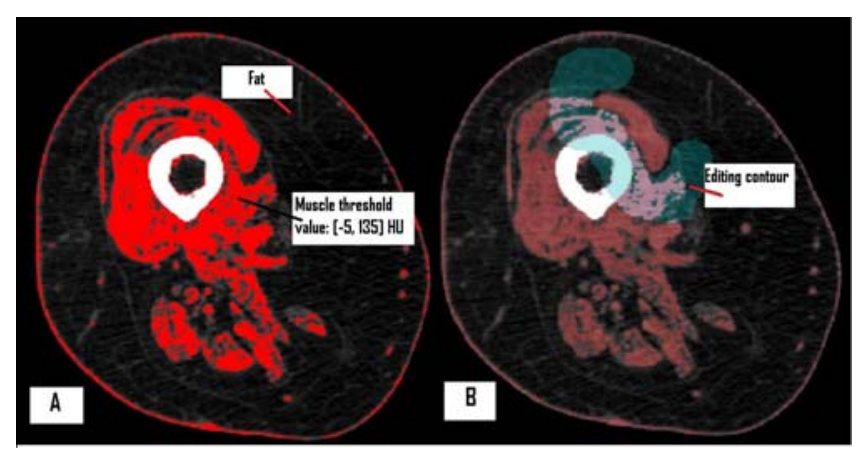

Fig 29 Segmentation process: Muscle thresholding (A), Manual editing of a region of interest (B)

The segmentation and 3-D modelling are particularly convenient techniques to study rectus femoris because of the definite shape kept even after a long term degeneration. Indeed in other muscles the degeneration process lead to a severe deterioration of the shape. In figure 1 are compared the 3-Dimensional models of right thighs in innervated (Fig. 28, A) with a 3-years denervated thigh (Fig. 28, B). The 3-D rendering show clearly how effects of degeneration in denervated muscles are dramatic: muscles in the lower limbs become very thin and not longer recognizable in their shape, rectus femoris instead remains recognizable among the muscles though is severely degenerated.

In this chapter the segmentation technique used to study rectus femoris will be described as well the methods used to measure volume, density and tissue composition. Finally the modelling approach and the implementation process to quantified geometrical changes will be presented in the chapter and fibre direction in rectus femoris will be visualised.

\subsection{Segmentation and 3-D visualization}

The image processing data source are CT scans images from the patients lower limbs taken with Spiral CT [Paragraph 2.1]. The scan data are imported in to special software called MIMICS [44] which allow processing and editing of 3-dimensional data. The goal is to study the behaviour of rectus femoris during the stimulation treatment, therefore the muscle must be first isolated from the surrounding and then reconstructed in three dimensions. Segmenting the patient data at different points of time will then allow rectus femoris volume, density and shape to be compared with very accurate monitoring of growth. The first step in the segmentation process is to establish a threshold which can properly represent the object of study. The data set after thresholding will display only certain information; the pixels having Hounsfield values (or more generally gray values when data aren't CT scans) within the defined interval are selected and underlined with colour while the others

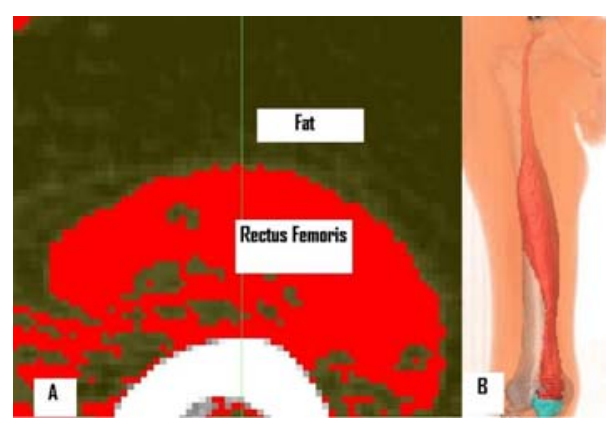

Fig 30 Cross section area of rectus femoris which shows the surrounding fat (A). Segmentation results; 3-D rendering of rectus femoris, femur and patella bones $(B)$.

having HU values outside the threshold remain black (Fig. 30A).

The threshold interval chosen to analyse rectus femoris is: $[-5,135] \mathrm{HU}$. This interval is as such defined because it must display muscle tissue and allow monitoring of changes, particularly the restorationdegeneration process. Within the selected interval the displayed pixels are representing both normal and degenerated muscles and also connective tissue but pure fat is excluded. In this way the surrounding fat in rectus femoris is automatically excluded from the segmentation mask (Fig. 30, A). After thresholding, the next step for the segmentation is to isolate rectus femoris form the other muscles. For this reason special editing tools are used. The process starts from a cross section where the muscle boundaries are well visible (usually in the middle, along muscle). A contour is drawn around the muscle and this shape projected to the next cross sections in both directions. If the contour well fit the new cross sectional area then it is projected unchanged forward to the next slide otherwise it is modified and projected ahead. The process continues until all cross sections displaying Rectus Femoris cross sections are edited (Fig. 30, B). The contours areas are then erased creating a gap between segmentation target and surrounding. Finally a new segmentation mask representing Rectus Femoris is created applying a region growing procedure [Paragraph 2.2] which creates a new mask separating the edited structure that is not longer connected to the surrounding. The result of the segmentation process is showed in figure $30, \mathrm{~B}$. The results from the $3-\mathrm{D}$ modelling are displayed in figure 31. The rectus femoris muscles are segmented and 3-dimensional modelled from beginning of stimulation treatment in 2003 until February 2008 (Fig. 31, A). An innervated muscle is also segmented as control to allow comparison and estimation of differences in shape and size (Fig. 31, B).

With this technique the degeneration effects on the muscle can be seen very clearly. Compared to a normal rectus femoris (Fig.31, B), the denervated muscles are 


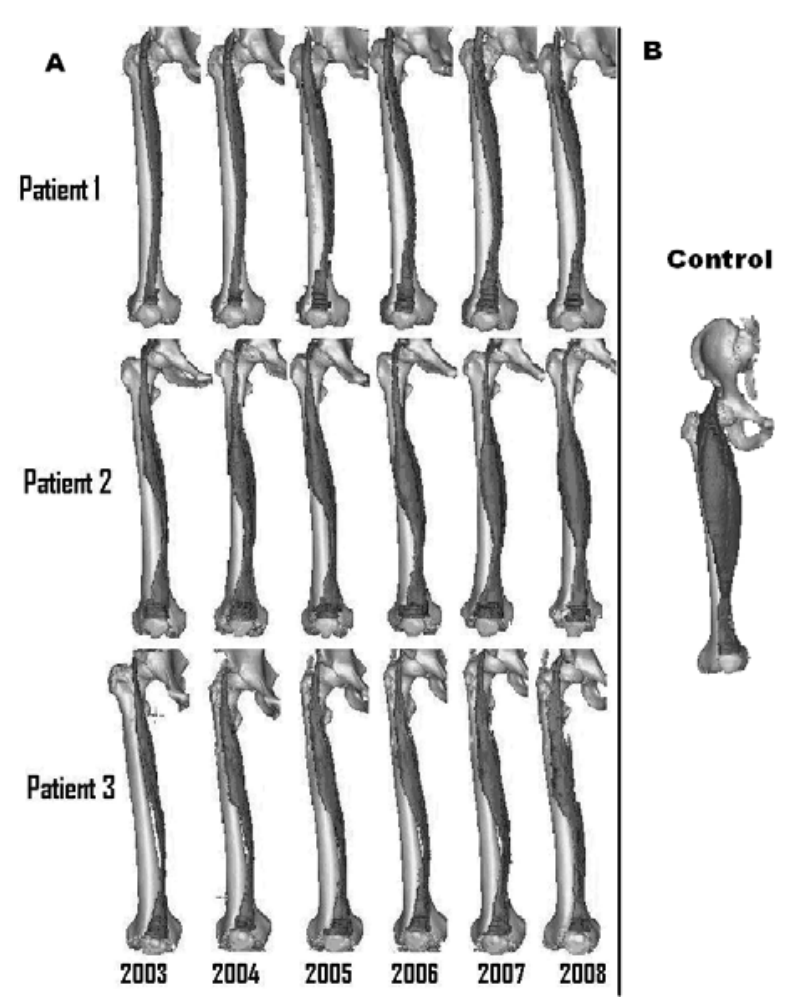

Fig 31 Rectus femoris 3-dimensional models front view of left leg: Denervated muscles of the three patients in the RISE project (A), innervated muscle (B)

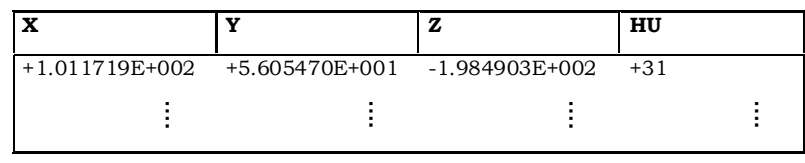

Table 6 First row of rectus femoris model text file. Every pixel in the rectus femoris volume is described by the spatial coordinate and the Hounsfield value.

definitely much thinner and with a shape which appear flaccid and degenerated.

The modelling work allows seeing changes induced through the stimulation treatment on whole muscle volume demonstrating incontrovertibly the stimulation results. For instance in patient 2 the changes are very remarkable; the 3-dimensional models show the progressive muscle growth during the stimulation and beside a remarkable difference in shape which in the late models appear starched and tonic. Some changes in shape are also noticeable from the other two patients but they are not as evident like in patient 2. However the 3-d model isn't only an object showing geometry and volume; it provides additionally information about geometrical distribution of pixels intensity which in our case are the HU values (Table 6). Using and developing these data, in the next paragraphs muscle
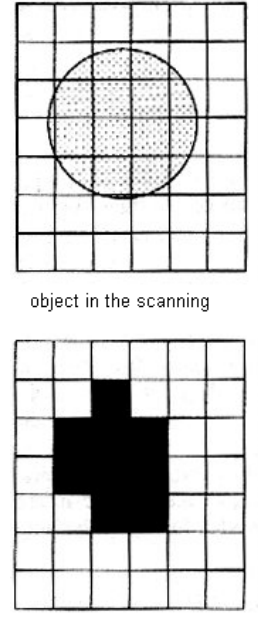

thresholded image

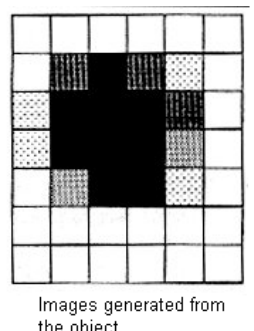

the object

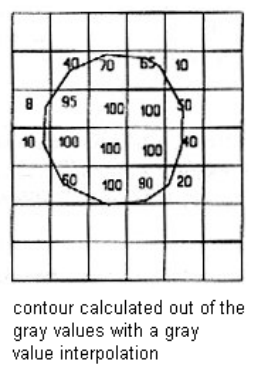

value interpolation
Fig 32 Partial volume effects; contour interpolation approach

density will be measured and tissue composition evaluated.

\subsection{Measurement of volume and density}

The modelling work is a unique way to visualize the influences of electrical stimulation on shape and geometry of denervated muscle. Beside the 3Dimensional file provide information on muscle density and volume which would be otherwise of difficult access. Muscle density and volume are immediately available from the segmentation object, these parameters depending from pixels number, size and intensity (generally gray value or HU value in CT scans).

\subsubsection{Measurement of volume}

In digital imaging, a pixel or picture element is the smallest piece of information in an image. A voxel instead, is a volume element representing a value on a regular grid in three dimensional space, the voxel data sets have a limited resolution, as precise data is only available at the center of each cell. This value of a voxel may represent various properties; in CT scans it accounts the opacity of material to X-rays (the Hounsfield units).

The volume in a 3-D file can be generally obtained through the voxel size and number of voxels in the 3-D object. In biological tissues this is more complicate and there are additional issues which must be taken into account. The image that results from the assumption that a voxel within a data set is occupied by one single material is an approximation of the reality because of real dimensions involved in biological tissue. As the voxels are not infinitesimal in size, the result is a measured value that is a mean value 


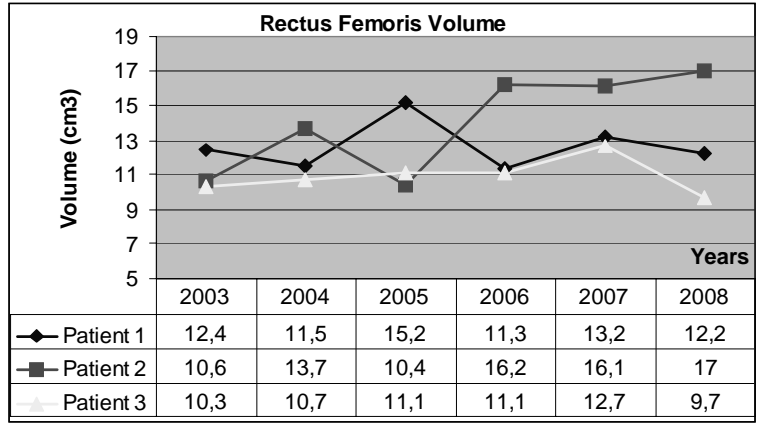

Fig 33 Rectus femoris: Volume trend

for the different materials (phenomenon known as partial volume effects, fig. 32). This will be the case especially for the voxels lying at the border of the object or voxels covering objects thinner than some of the voxels dimensions. This problem can be solved assigning mean values to these voxels and using interpolation algorithms.

In Mimics which we use for segmentation and modelling work, there are two different algorithms that can be used to compensate partial volume effects. The first is called contour interpolation and work to compensate partial volume effect on 2D (in each cross section) and the second is called gray value interpolation which uses a different approach taking into account also the neighbouring voxels above and below. The latter algorithm is more accurate but tends to result in a noisy 3D model which makes it less useable for medical images. By taking into account the partial volume effect also the 3D model rendering will be more accurate.

In this work the contour interpolation algorithm was used to visualize the 3D model of rectus femoris and the gray value interpolation algorithm to calculate and measure the muscle volume.

The results from the measurement of rectus femoris volume are gathered in Figure 33. Muscle volume changes remarkably during the stimulation trial in all of the three patients. The changes are alternately positive and negative due the patient compliance and other clinical factors which have been influencing the electrical stimulation treatment.

As seen in figure 31, rectus femoris volume from patient 2 show the best results; indeed the muscle volume increases of $60 \%$ (from $10,6 \mathrm{~cm} 3$ in 2003 to 17 $\mathrm{cm} 3$ in 2008). It is important to remark that the muscle grow process is rather fragile, indeed the volume drops in autumn 2005 (-24\%) because of bed compliance and interruption of the stimulation treatment in the previous period (patient compliance tables in paragraph 2.3).

In patient 1 the muscle volume goes up and down during the monitoring period, demonstrating the
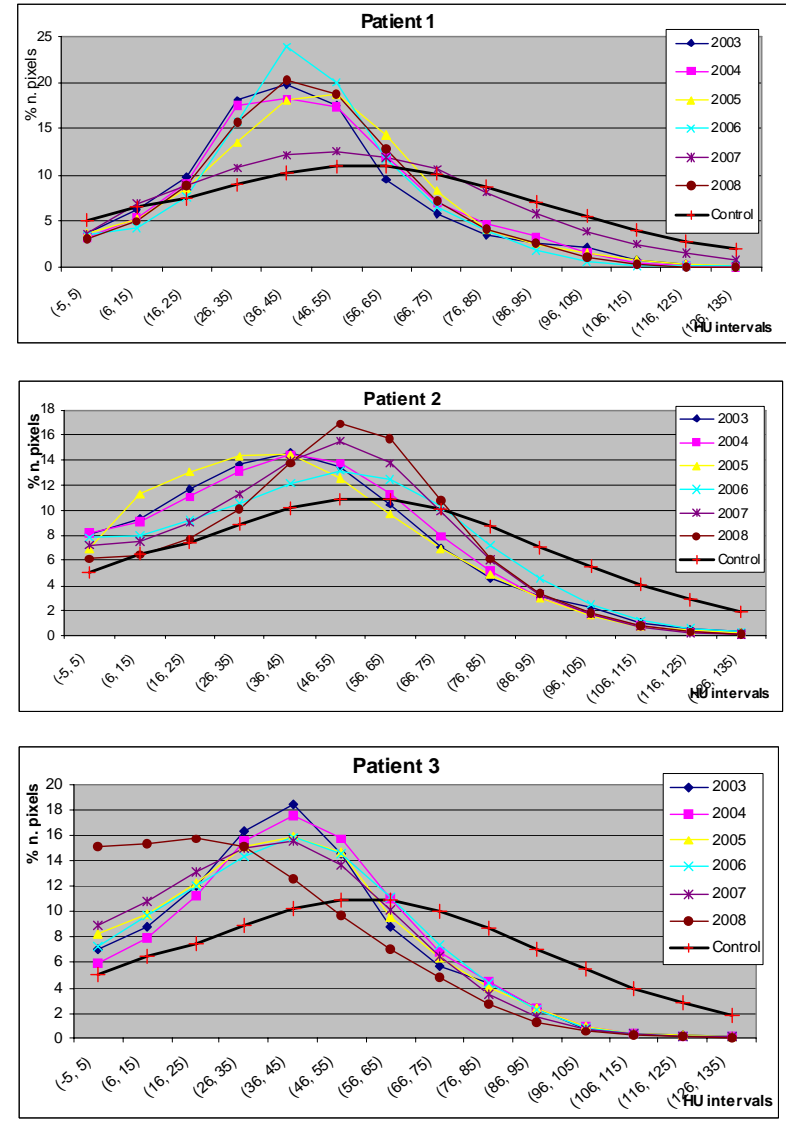

Fig 34 Density distributions in rectus femoris muscle during the stimulation trial

sensitivity to the stimulation treatment but also showing the fickleness of the patient in performing the treatment. Anyway the muscle didn't degenerate further maintaining substantially the same initial volume (12,4 cm3 in 2003 to $12,2 \mathrm{~cm} 3$ in 2008).

Finally patient 3 which seem not to be responding (or responding minimally) to the treatment until 2006, then the volume increase remarkably from 2006 to 2007 $(+14 \%)$ growing of $23 \%$ since the beginning of the clinical trial in 2003. Unfortunately the patient incurs in a new clinical problem (patient compliance tables in paragraph 2.3) in early 2008 and therefore he stops the stimulation with consequent fall in of muscle volume.

\subsubsection{Measurement of density}

Rectus femoris density distributions are calculated at different point of the time during the stimulation trial and are compared with the control distribution. The control is a healthy rectus femoris with a volume of 41,7 cm3 (Fig. 34, B, the control thigh ).

To display the muscle density the pixel intensity within the rectus femoris volume is required.

The muscle density is evaluated by monitoring the number of pixels in the Hounsfield interval [-5 to 135] $\mathrm{HU}$. To visualize and analyse the distribution, the $\mathrm{HU}$ interval is divided in 14 sub intervals with $10 \mathrm{HU}$ 


\section{D Modelling and monitoring of denervated muscle under FES}

European Journal Translational Myology - Basic Applied Myology 2011; 21: 31-94

values each and changes are measured between these HU intervals and not between individual values. Indeed compare occurrences between individual HU numbers from different data set isn't a reliable measurement; the same tissue maybe coded with different $\mathrm{HU}$ value depending from the scanning device used, the calibration, and the relative position of the biological tissue with the scanner gantry (paragraph 2.1.4). Therefore the comparison between HU intervals is more meaningful way to monitor changes in the muscle density.

To allow a better comparison between data set, the numbers of pixels in each interval are displayed in percentage then the subtended areas in the distributions are the same and the changes in tissue composition are underlined.

Furthermore, the number of pixels in the data set depends from the used scanning protocol, in particular from two parameters: field of view and slide increment (Paragraph 2.1.5). These parameters set pixel size and total number of pixels in the data set, therefore the same region of interest maybe displayed with different number of pixels if these parameters are different.

The rectus femoris density distributions from the three patients are gathered in figure 4.7: the number of pixels (in percentage) is on the ordinate axes and the HU intervals on the abscissa. Muscle density distribution from 2003, 2004, 2005, 2006, 2007 and 2008 are displayed in the same panel and compared with the control.

The control distribution shows that in normal muscle most of the Hounsfield values are distributed between 26 and $85 \mathrm{HU}$ with the mean value of 54,5 HU (control distribution in figure 34 ). The situation is completely different in degenerated muscles where, depending from the degree of degeneration, the muscle became less and lesser dense. In 2003 before the patients started to stimulate rectus femoris mean density values were the following: patient 1 (one year denervation)

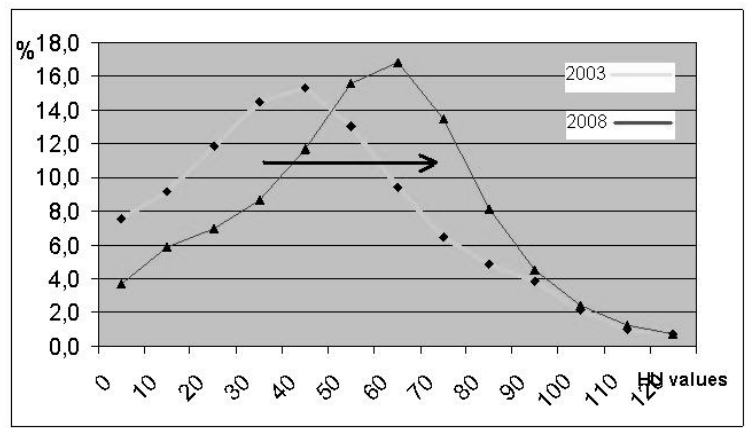

Figure 35 Density shift in patient 2 rectus femoris

In patient 3 the density distributions don't change remarkably during the stimulation years (such as in the volume in figure 34), only in 2008 the changes is sensible and the distribution appear completely different from the others because of a severe edema which cause an abnormal presence of liquids in the muscle.

\subsection{Muscle tissue analysis}

The degeneration process changes completely the muscle physiology and the fiber structure; muscle fiber which normally looks very organized and structured (Fig. 36, A), became completely disarranged and chaotic in long term denervated musculature (Fig. 36, B). Fat and connective tissue replace and mixes within the muscle fiber varying completely the tissue consistency.

During the project RISE histological examinations are made to study the muscle fiber composition and the physiological mechanisms involved in degeneration and regeneration process. In fact, as it is shown in figure $36, \mathrm{C}$, the denervated ultra muscular structure seems to reorganises again as effect of the electrical stimulation treatment. This approach gives important indication on the changes occurring in the muscle

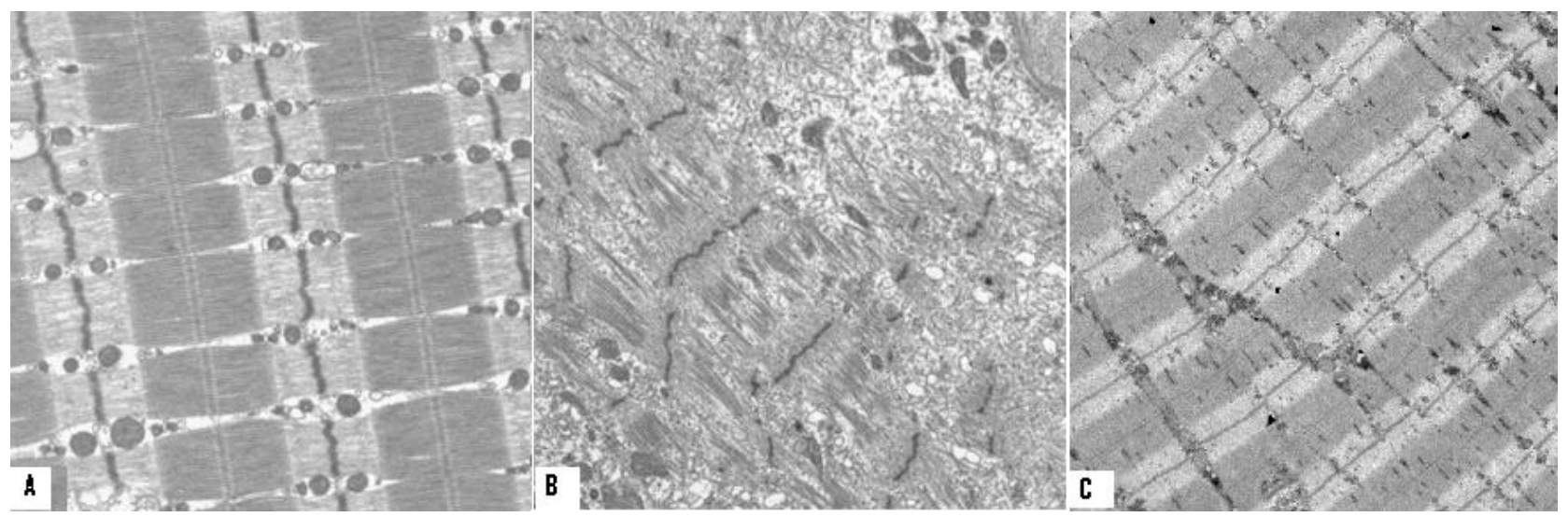

Fig 36 (A) Normal ultra muscular structure. (B) 2 Years denervated muscle. (C) 4 Years denervated, 2 years stimulation. Pictures from CeSi Center for Research and Ageing, University G. D'Annunzio Chieti (Italy) 

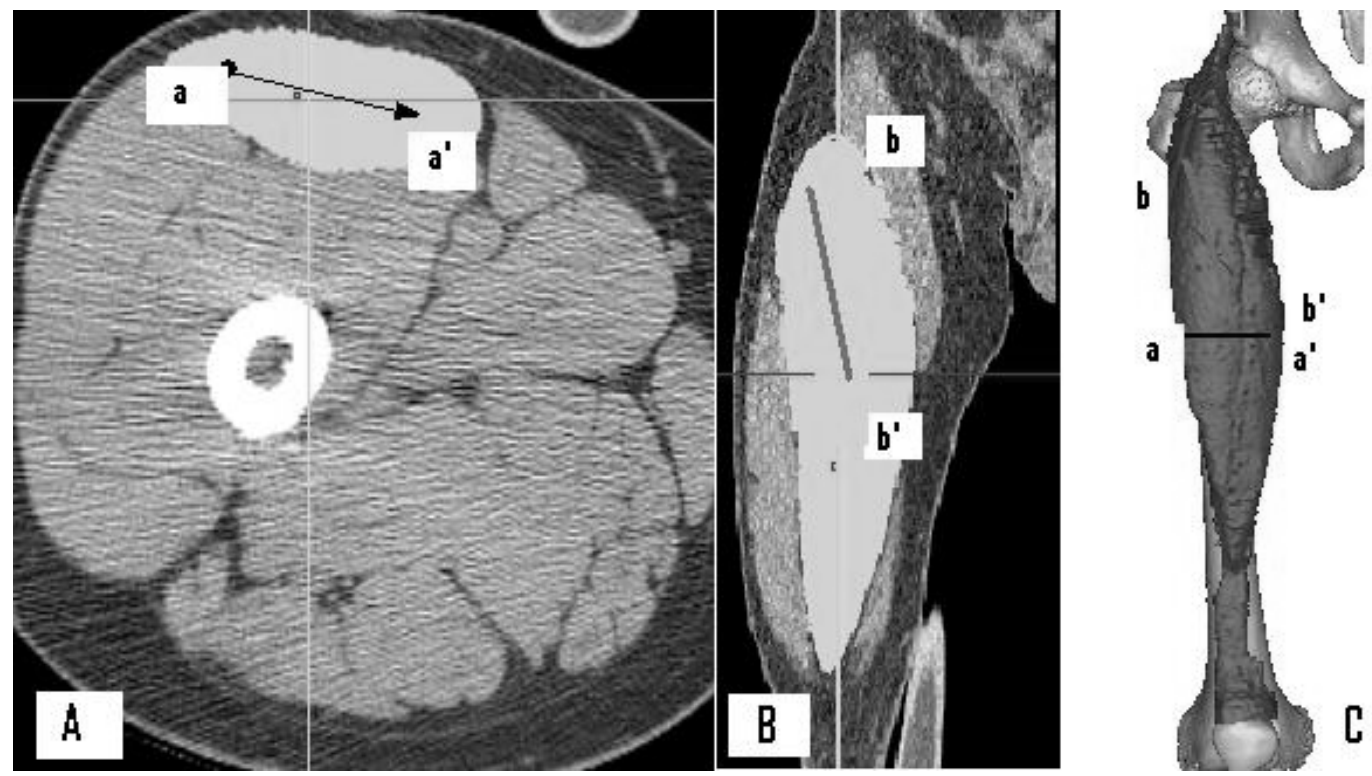

Fig 37 (A) Profile line on the axial plane. (B) Profile line on the coronal plane. (C) Profile line plotted on the 3-D rectus femoris model

Research and Ageing, University G. D'Annunzio Chieti (Italy)

\subsubsection{Profile line method}

A profile line can be defined as a line describing certain, local characteristic of the region where it is plotted. In our case the region are the muscles and the characteristic we want to measure is the local Hounsfield value. Practically the profile line is traced directly on the CT slides in one of the three planes and through the direction of interest as it is showed in

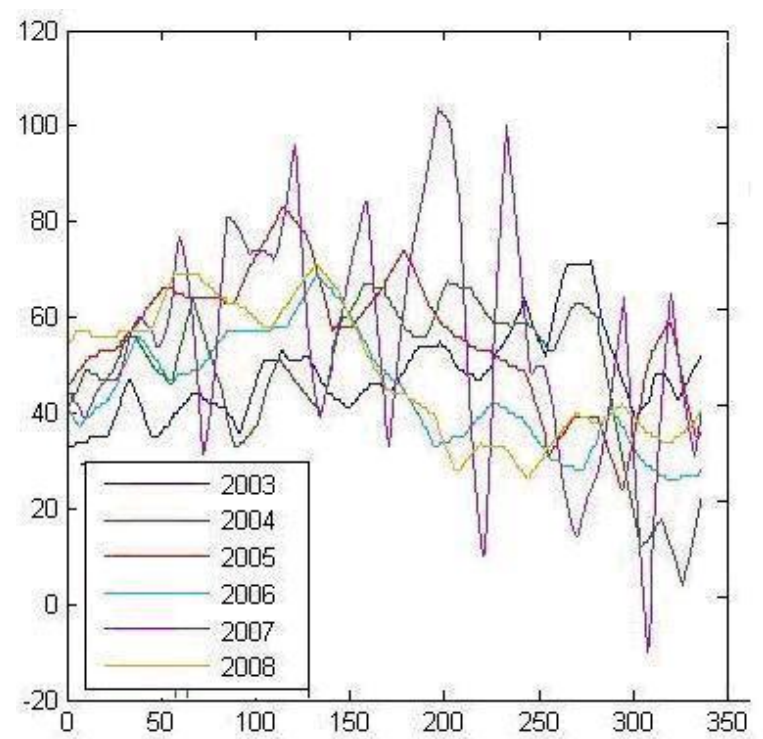

Fig 38 Profile line comparison for patient 3 (a-a) segment). figure 37. The $\mathrm{HU}$ values intercepted along the segments provide local information on muscle density. Therefore this approach add to the density distribution analysis described previously the local tissue characterisation along the profile line. The muscle density will be studied longitudinally and transversally to the muscle fiber direction.

In our analysis two profile lines for each data set are plotted: one in the middle of rectus femoris along the muscle width (Fig. 37, A) and the other approximately along the muscle fiber direction (Fig. 37, B). In figure 37 , C the two profile lines are displayed on the 3Dimensional rendering of rectus femoris. The profile line analysis will be performed using two profile lines having different length: the profile line measuring the tissue composition on the axial plane is $35 \mathrm{~mm}$ (a-a) segment), the profile line on coronal plane is $180 \mathrm{~mm}$ long (b-b' segment).

These profile line length are established to suit the dimensions of the target muscles to be investigated.

The results from the profile lines analysis (a-a' segment) for patients 3 are displayed in figure 38 . The different data sets are compared; HU values on the ordinate axes and position along the line on the abscissa. The resulting signals appear noisy, the profile lines show the different signal amplitude but the differences in tissue composition between data set are of difficult to interpret. To improve this analysis a method ad hoc is developed using Matlab. The objective is to code the information contained in the profile line in order to make them more understandable. The Hounsfield values from the profile line are sorted in three groups: one representing high dense muscle fiber, the second representing the low 

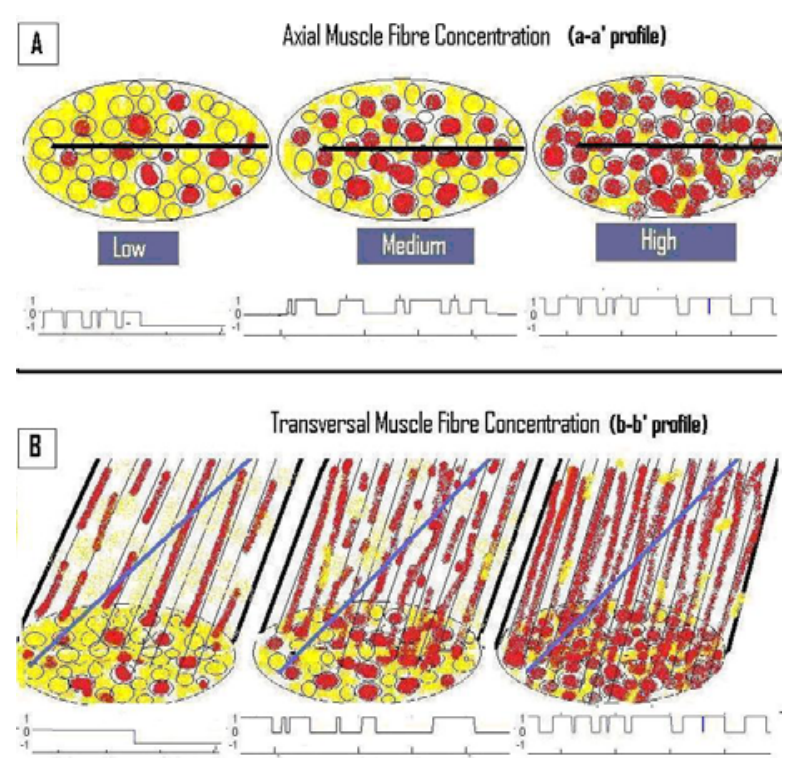

Fig 39 Example of central lines analysis with three different muscle density and tissue composition. (A) Axial analysis, coding of aa' segment. (B) Longitudinal-transversal analysis, coding of $b$-b' segment

dense muscle fiber which are mixed with connective tissue and fat and then the third group where the content of fat is predominant in the muscle and therefore the overall density is very low.

The three groups numerically are as following coded:

- 1: HU $>=55$ (High muscle density)

- 0: $10=<\mathrm{HU}<55$ (Medium muscle density)

-1 : HU $<10$ (Low muscle density)

The monitoring technique is modelled in figure 39: the profile lines intersect the muscle fibres in the axial and in the coronal plane, three different density

\subsubsection{Profile line results}

The results from the profile line analysis are displayed in figure 40; instead of noisy looking signals the new profile lines provide only three levels $(1,0$ and -1) accounting the differences in tissue consistency along and through the muscle fibres. With this method the tissue characterisation and the comparison between data is easier. The charts on the left column shows profile lines from the axial plane (a-a' segment, $35 \mathrm{~mm}$ length), the charts on the right column shows the profile lines on the coronal plane, transversal to the fiber direction (b-b', 180mm length). The control profile lines are showed in the bottom of figure 40: the density distribution along these lines isn't constant as one would expect in a normal muscle but varying considerably both along the width and through the length. Anyway the muscle tissues are mostly coded with 1 alternating with short regions coded with 0 .

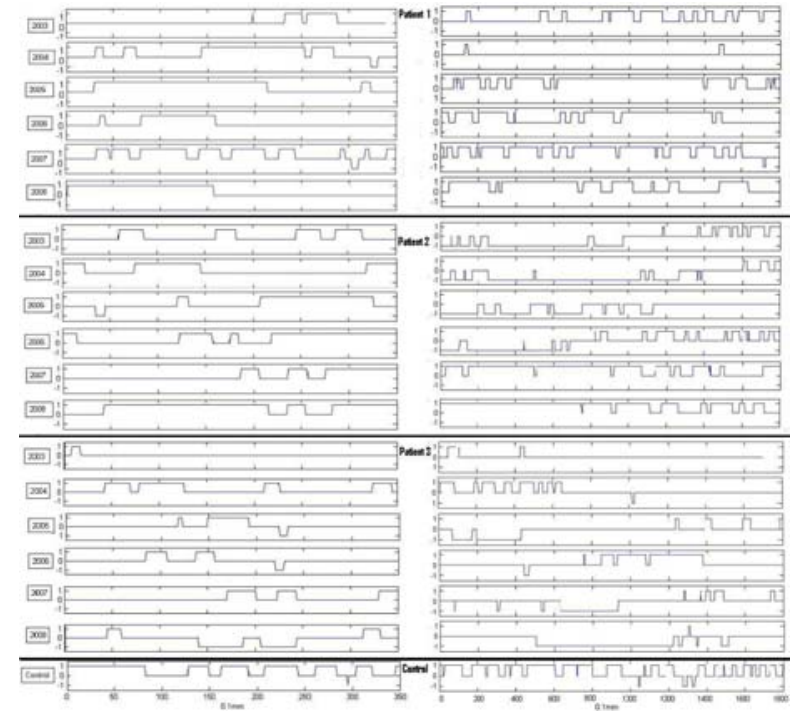

Fig $40 \quad a-a$ ' axial profile lines (Left). b-b' transversal profile lines (Right)

Only through the transversal direction and along a very short tracts (in the order of $0,1 \mathrm{~mm}$ long) the muscle tissue assume the value -1 .

In patient 1 , the muscles are not severely degenerate in 2003 and therefore in both sets of profile lines there aren't segment on the tissue with very low density (coded with -1). Anyway the muscle looks quite different in 2003 and 2004 compared to the control; there are large areas of low-medium density (coded with 0) which indicates intramuscular fibres space corrupted with fat and connective tissue. Improved profiles are measured in 2005 and 2006 but the best profile is shown in 2007 where, compared with the control, the muscle looks almost dense as normal both on the axial and on the transversal profiles.

In patient 2 the initial situation is very bad; the muscles are severely corrupted by fat and connective tissue. Especially on the transversal profiles the density is very low with large signals segments coded with -1 . The situation in the muscle can be imaged as in figure 10-left, where the intramuscular fiber space is filled with fat and the muscle fiber is segmented through its length. The muscle condition start to improve in 2006 and in 2008 the profile measurement appears completely different compared to the year 2003. In fact the muscle became a lot denser and the areas coded with -1 disappearing completely from the distribution. Finally, patient 3 which show in 2003 a low density distribution along the whole signal for both profiles due the long term degeneration. However the situation improves slightly in 2004 and again in 2006 after a partial regression in 2005. The situation remains almost unchanged in 2007 but dramatically change in 


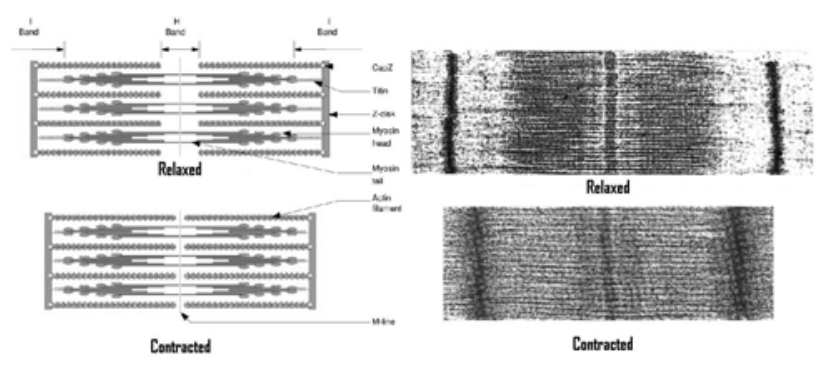

Fig 41 Geometrical changes during muscle contraction. (Left) Schematic actin and myosin cross-bridge cycling. (Right) Fiber contraction from electrical microscopy

where the muscle density collapses because of clinical problems (patient compliance, paragraph 2.3).

\subsection{Geometrical changes in rectus femoris}

A muscle contraction (or muscle twitch) occurs when a muscle fibre generates tension through the action of actin and myosin cross-bridge cycling (see figure 41). While under tension, the muscle may lengthen, shorten or remain the same. Though the term 'contraction' implies a shortening or reduction, when used as a scientific term referring to the muscular system contraction refers to the generation of tension by muscle fibers with the help of motor neurons.

Therefore changes in shape and geometry are inherent to normal muscle contractile activity. In denervated muscles the ability to spontaneously lengthen or shorten due to contraction is interrupted. The contractile activity can be partially activated only through the induced external stimulation.

In denervated muscle, degeneration causes loss of

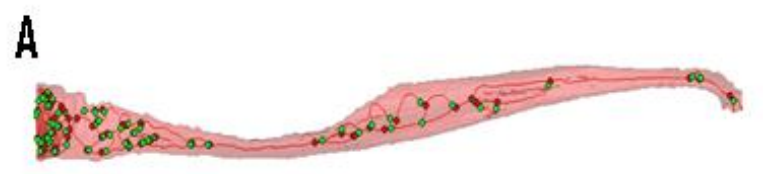

B

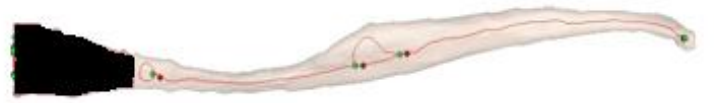

Fig 43 Muscles before and after thresholding and achieving the same length for all muscles in the same dataset. (A) Before. Muscle with many central lines, 32 extra branches. Patient 3, year 2003. (B) After. One central line, 1 extra branch (in the middle). Patient 3, year 2003

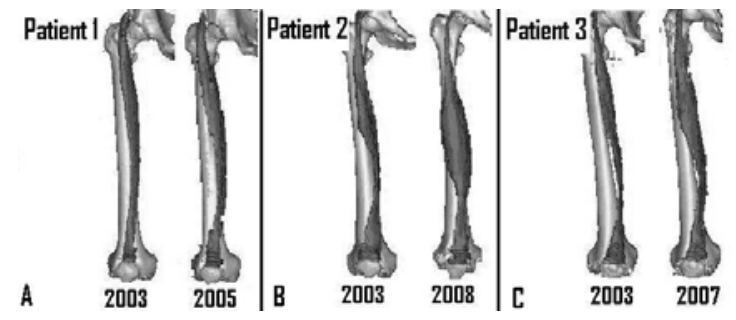

Fig 42 Changes in muscle shape. The rectus femoris displayed are from data sets where the difference in shape was on the 3-D model more remarked: (A) 2003-2005 patient 1, (B) 2003-2008 patient 2, (C) 2003-2007 patient 3.

muscle volume and density but also some geometrical changes. In our 3-dimensional modelling the tendency of the denervated rectus femoris to degenerate is quite visible also in their shape; the muscle appears flaccid and deformed especially in the lower part as showed in figure 42. As effect of the stimulation treatment, beside the volumetric and density grow, rectus femoris become more straight and tonic (especially visible in figure 42, B). Therefore induced contraction activity and changes in the muscle shape seem to be related with each other and with the restoration process.

The objective of this work is to quantify changes in muscle shape and correlate them with the restoration of muscle function and contractile activity. Preliminary work was done and published (Paragraph 3.2) using a 2-Dimensional approach; as following a different method based on the use of central line and 3Dimensional data is introduced and explained.

\subsubsection{Central line method}

A normal rectus femoris has almost a straight shape as can be seen in the figure $4 \mathrm{~B}$ while degenerate muscle tend to bend as consequences of missing contractile activity. To determine and quantify the geometrical shape in rectus femoris a central line through the muscle volume is defined and the curvature values along the line calculated. Finally the changes in muscle shape are measured comparing at different point of time the curvature values.

The central line is a CAD tool developed by Materialise [44] and develops basically by interpolating central points through the object cross sections. According to the algorithm strategy used by Materialise the central point in each cross section can be unique or multiple depending from the number of edges and from the object density. With our initial set up, the muscles are displayed with HU values between -5 and 135 and the muscle contains many central lines (figure 43, A). The reason for this is that connective tissue and fat are not displayed in the 3D object (because they are outside the threshold) although they are present inside the muscle and especially in a 
3D Modelling and monitoring of denervated muscle under FES

European Journal Translational Myology - Basic Applied Myology 2011; 21: 31-94

\begin{tabular}{|c|c|c|c|c|c|c|}
\hline \multicolumn{7}{|l|}{ Muscles information } \\
\hline & 2003 & 2004 & 2005 & 2006 & 2007 & 2008 \\
\hline Original muscle length [mm] & 487,04 & 497,75 & 515,44 & 524,07 & 513,82 & 506,62 \\
\hline Short muscle length [mm] & 434,01 & 434,03 & 434,30 & 434,58 & 433,91 & 434,17 \\
\hline Error [mm]* & 2 & $+0,02$ & $+0,29$ & $+0,57$ & $-0,10$ & $+0,16$ \\
\hline Error [\%]* & 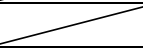 & 0,005 & 0,067 & 0,131 & $-0,023$ & 0,037 \\
\hline Short / Long [\%] ${ }^{\star *}$ & 89 & 87 & 84 & 83 & 84 & 86 \\
\hline Central Line length [mm] & 435,87 & 456,45 & 425,32 & 414,78 & 404,65 & 416,22 \\
\hline
\end{tabular}

\begin{tabular}{|l|l|l|l|l|l|l|}
\hline \multicolumn{7}{|l|}{ Patient : 2} \\
\hline Muscles information & 2003 & 2004 & 2005 & 2006 & 2007 & 2008 \\
\hline & 508,59 & 512,15 & 526,65 & 547,98 & 518,15 & 518,41 \\
\hline Original muscle length [mm] & 421,04 & 421,41 & 421,49 & 421,35 & 420,57 & 421,50 \\
\hline Short muscle length [mm] & & $+0,37$ & $+0,45$ & $+0,31$ & $-0,47$ & $+0,46$ \\
\hline Error [mm]* & & 0,088 & 0,107 & 0,074 & $-0,112$ & 0,109 \\
\hline Error [\%] & & 82 & 80 & 77 & 81 & 81 \\
\hline Short / Long [\%]** & 83 & 82 & 411,79 & 385,28 & 432,02 & 410,85 \\
\hline Central Line length [mm] & 419,18 & 391,34 & 40 & & &
\end{tabular}

\begin{tabular}{|l|l|l|l|l|l|l|}
\hline \multicolumn{7}{|l|}{ Patient : 3} \\
\hline Muscles information & 2003 & 2004 & 2005 & 2006 & 2007 & 2008 \\
\hline & 480,73 & 483,61 & 520,19 & 494,50 & 512,67 & - \\
\hline Original muscle length [mm] & 433,00 & 433,20 & 432,98 & 433,57 & 432,78 & - \\
\hline Short muscle length [mm] & & $+0,20$ & $-0,02$ & $+0,57$ & $-0,22$ & - \\
\hline Error [mm]* & & 0,05 & $-0,005$ & 0,13 & $-0,05$ & - \\
\hline Error [\%]* & 91 & 90 & 83 & 88 & 84 & - \\
\hline Short / Long [\%]** & 434,43 & 441,19 & 422,58 & 429,79 & 429,13 & - \\
\hline Central Line length [mm] & & & & & & \\
\hline
\end{tabular}

Table 6 Patients 1, 2, and 3.

* 2004, 2005, 2006, 2007 and 2008 compared to the reference length, 2003.

** Short muscles percentage of long muscles

degenerated muscle. Therefore the mask representing rectus femoris contains gaps in these areas which generate multiple central points and then central lines. To allow an accurate analysis of the shape a single central line is wanted and therefore it is necessary to fill in these gaps in order to get a homogeneous mask. Thresholding is used for this purpose. A new threshold is established in order to include all the tissues present in the muscle volume. The HU interval value chosen is
$[-300,+300]$, with this threshold every pixel inside the boundary of RF is marked, including pixel representing connective tissue or fat. This new mask contains information about the original shape of the muscle and it is homogeneous and thus has few central lines. When this homogeneity is achieved the muscle contains one main central line, that passes through the whole muscle and is therefore suitable to use to describe changes in shape of the muscle as a whole. The length of these 
A
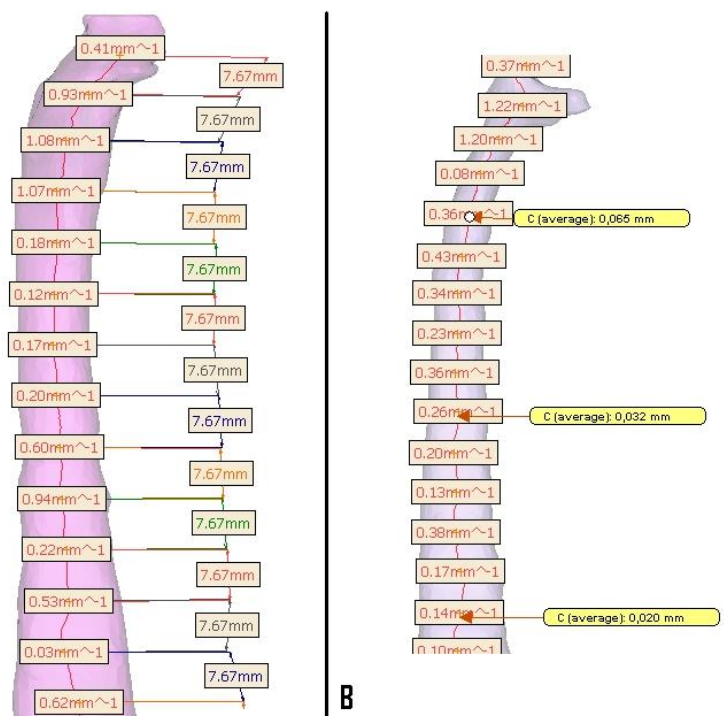

Fig 44 (A) Patient 2, year 2004. Example of how the $50 C$ (curvature) values were sampled on regular distances on the central line. (B) Mean $C$ values for groups 1-3. Patient 2, year 2008.

main central lines lies between 380 - $460 \mathrm{~mm}$ depending from the patient, few extra branches are still obtained even with the new threshold but they are very short and not used to quantify changes in the shape of the muscle (figure 43, B).

Excluded from the analysis is the muscle-tendon segment which connects rectus femoris to the patella (figure $43 \mathrm{~B}$, marked black). There the muscle is far from being homogeneous because bones and tendons tissue mixes with the muscle having as consequence high density areas not included in the threshold which then forms several extra central lines not useful for our investigation. The central lines from the three patients are calculated and displayed in Appendix 2; the numerical measurements on the central line are gathered in Table 6.

\subsubsection{Curvature index}

The central lines are compared to quantify the muscle shape changes using the centerline curvature index (C). The curvature index measures the curvature at a specific point on the central line, as the curvature of the line increases the higher is the $\mathrm{C}$ value. The curvature $(\mathrm{C})$ of a curve is the magnitude of the rate of change of the unit tangent $(\mathrm{T})$ with respect to the arc length (s) along the curve:

$$
C=\left\|\frac{d T}{d s}\right\|
$$

For each rectus femoris 50 curvature values are sampled, on regular distances, on the central line.

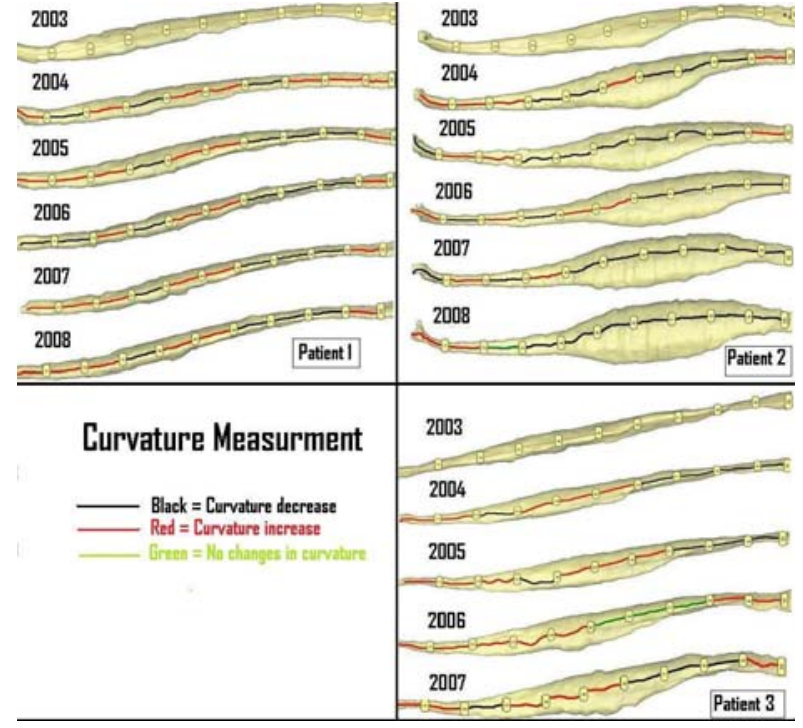

Fig 45 Curvature changes; qualitative results are displayed directly on the centreline. The central line segments have different colour according to the results from the curvature ratio: Black curvature decrease, red curvature increase and green there are no changes.

The curvature index measures the curvature at a specific point on the central line, but a single point could fall on a spot where there is a discrete curve that does not really represents the overall shape of the muscle in that place. For this reason the $50 \mathrm{C}$ samples are divided into 10 groups (5 $\mathrm{C}$ values in each group) and a mean value in each group calculated (Fig.17B). In this way the curvature values represent not a point but a segment of the central line.

The approach that was used to compare the central lines through its average curvature values was to use ratio calculations. This is done by setting as fixed denominator the 10 mean curvature values from year 2003 (a1, a2...a10) then it is possible to estimate whether the curvature increases or decreases by calculating the ratios 2004/2003 (b1/a1, b2/a2...b10/a10), 2005/2003 (c1/a1, c2/a2...c10/a10) etc.. If the ratio is higher than 1 then there is an increase in curvature at the specific segment, if otherwise the ratio is lower than 1 the curvature is decreasing along the specific central line segment. As the ratio value is higher the higher is the increase in curvature between the specific years and vice versa. The computational work and the resulting tables are gathered in Appendix 1.

The results from the curvature analysis are showed more simply in figure 45 where the qualitative results are displayed directly on the centre line. The central line segments have different color according to the results from the curvature ratio: 


\begin{tabular}{|c|c|c|c|c|c|c|c|c|c|c|c|c|}
\hline \multicolumn{13}{|c|}{ Patient : 2} \\
\hline \multicolumn{13}{|c|}{ Ratio results } \\
\hline $04 / 03$ & \multirow{5}{*}{$\frac{\mathscr{n}}{\bar{\Omega}}$} & 1,40 & 1,86 & 1,55 & 0,58 & 0,81 & 1,10 & 0,25 & 0,93 & 0,50 & 1,56 & \multirow{5}{*}{$\frac{\frac{\pi}{\bar{\Phi}}}{\frac{\pi}{\pi}}$} \\
\hline 05/04 & & 0,54 & 0,62 & 0,85 & 0,99 & 1,05 & 0,58 & 2,14 & 0,34 & 0,58 & 1,08 & \\
\hline $06 / 05$ & & 1,67 & 0,85 & 1,01 & 1,57 & 1,64 & 3,65 & 0,26 & 2,77 & 0,70 & 0,54 & \\
\hline $07 / 06$ & & 0,45 & 1,69 & 0,68 & 1,87 & 0,62 & 0,04 & 2,25 & 0,77 & 2,81 & 0,89 & \\
\hline $08 / 07$ & & 2,14 & 0,91 & 1,12 & 0,41 & 1,00 & 7,20 & 0,59 & 0,64 & 0,96 & 0,68 & \\
\hline
\end{tabular}

Table 7

Results for Patient 2 in more detail, average $C$ value for each year is compared to the year before. Red numbers represent an increase in $C$, black a decrease and green represent no change in $C$.

- bn/an $<1$ then the segment $\mathrm{n}$ of the central line $\mathrm{b}$ is coded with Black

- $b n / a n>1$ then the segment $n$ of the central line $b$ is coded with Red

- $\mathrm{bn} / \mathrm{an}=1$ then the segment $\mathrm{n}$ of the central line $\mathrm{b}$ is coded with Green

The central line segments are displayed in black when the curvature decreases, in red if it increases and finally in green if no change in curvature are measured. The overall pictures show that there is a general decrease in curvature, between the years 2003 and 2008 , for all the patients. This decrease is concentrated in the lower (distal) part of RF where the muscle bends the most as effect of degeneration. The results from the curvature analysis correlate to the other measurement performed on muscles. Indeed the decreases of curvature are most obvious in Patient 2 and the improvements in shape seem to progress side by side with muscle and density growth.

In patient 1 the curvature decrease occurs in groups 7-9 (more close to the patella, figure 4.18) while increase of curvature are measured in centre. Also the changes in curvature for patient 1 seems to be in line with other measurement; the volume for example don't change very much during the years expect in 2005 where there is a remarkable increase, thus as well in the curvature analysis can be observed a sensible increase in 2005 compared with the previous year.

Also in patient 3 is important to underline that the best results in terms of curvature is measured in 2007 in the same year where also the muscle volume increases the most $(23 \%)$.

The results demonstrate that these shape changes, i.e. decrease in curvature, are local and occur mostly in the lower part of rectus femoris closer to the patella. Beside the data, compared with other measurement, shows that curvature decrease as the muscle restores. To underline the correlation between muscle grow and straightening, in table 4.5 the curvature ratio values during the 5 years stimulation are gathered for patient 2. The ratios are between consecutive years i.e. $2004 / 2005,2006 / 2005$ and so on, therefore increase in curvature (or decrease) from one year to the next are quantified. Except for the ratio 2006/2005, the data show a progressive curvature decrease, in line with the progressive increase of muscle volume and density.

The central line measurement and curvature analysis are validated tools to quantify geometrical changes and the diminishing in curvature seems effectively correlated to the muscle growth.

\subsection{Modelling muscle fiber direction}

This chapter concludes with a preliminary study on

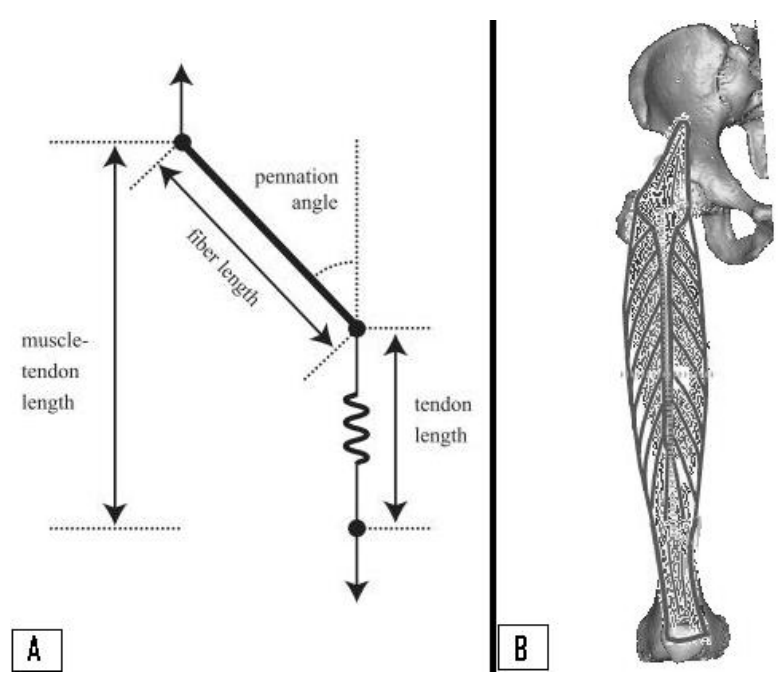

Fig 46 (A) 2-Dimensional representation of muscle architecture; the model represents tendon length, muscle-fiber length, and pennation angle. (B) Fiber direction in rectus femoris 


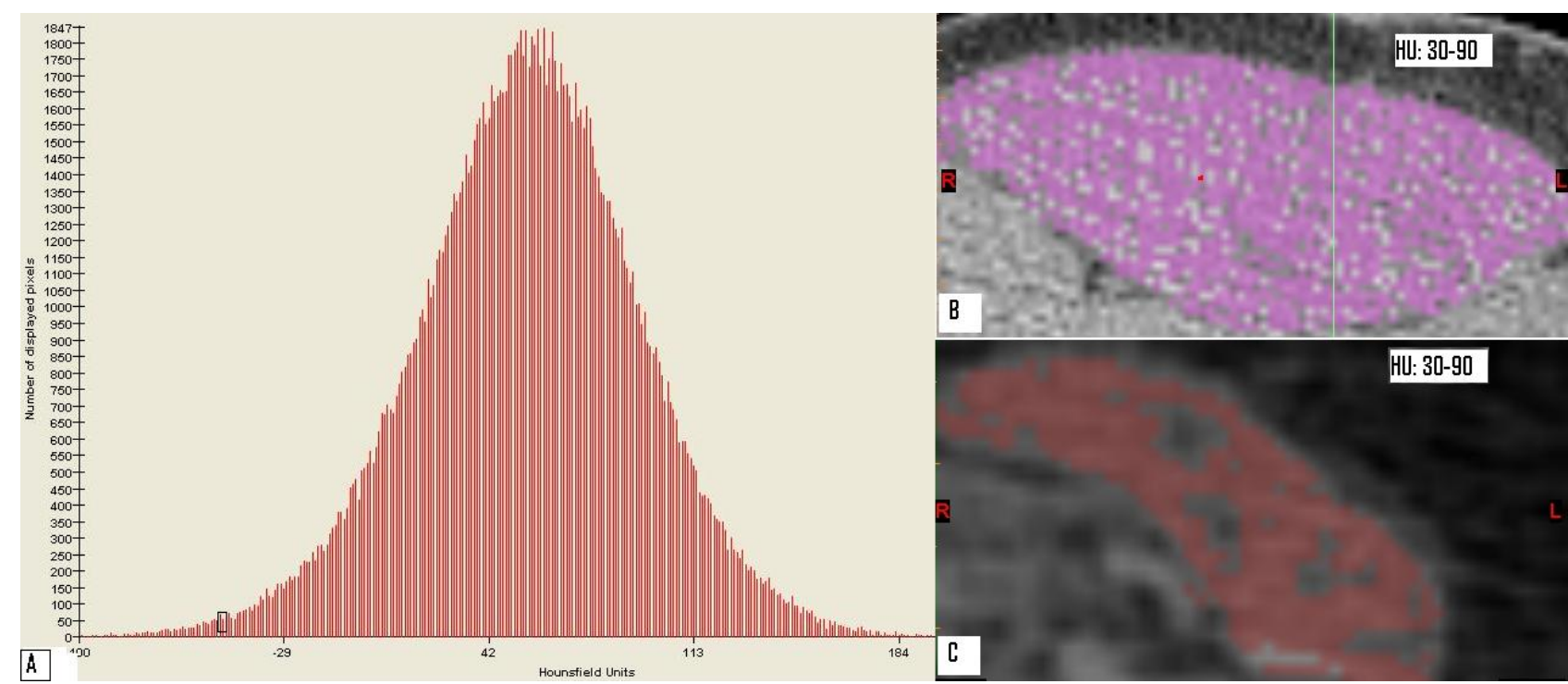

Fig 47 Density distribution in a normal rectus femoris (A). HU values between 30 and 90 displayed on a Rectus Femoris cross sections, in normal subject (B), in a denervated subject (C).

modelling of rectus femoris fiber direction.

The modelling of muscle fiber direction is in general a topic of interest in many biomechanics research, since the organization of muscle fibers and tendon in a muscle-tendon architecture is directly connected to the muscle's ability to generate force and movement. Muscle force is in fact proportional to physiologic cross-sectional area (PCSA) while the muscle velocity is proportional to muscle fiber length [47].

A simplified, two dimensional representation of muscle architecture which is commonly used in models of the musculoskeletal system is displayed in figure 4.19A. The model represents the tendon length, the muscle-fiber length, and the pennation angle. This model assumes that all fibers have the same length, that all fibers shorten by the same amount, and that the aponeurosis has the same properties and behaviour as the external tendon.

In quadriceps muscles the architecture is rather complex; the fibers of the rectus femoris muscle follow three-dimensional trajectories and there are two long aponeuroses distal-posterior and proximal-anterior aponeuroses within the muscle (Fig. 46,.B).

In denervated muscle the fiber direction is not known and a model not yet available. Though is presumable that the restoration process which restores part of the force capability will influence the fiber direction as well. The objective of this work is to preliminary model the muscle direction in denervated muscle and in denervated muscle which are restoring.

\subsubsection{Denervated fiber direction: density tracker}

To estimate the muscle fiber direction in denervated muscle a method based on the tracking of muscle density is developed. The muscle fibres are bundled together and surrounded with connective tissue, other tissue such as fat are also present within the muscle but in very small amount. The density in normal muscle is distributed on a wide range of $\mathrm{HU}$ values -100 up to $258 \mathrm{HU}$ (Fig. 47, A), though most of the tissues have HU values between 30 and 90 and the muscle appear in this interval basically homogenous and uniformly dense (Fig. 47, B).

In denervated muscle the degeneration process literally transform the muscle physiology; the muscle is not longer spontaneously contracting and the tissue composition and architecture is modified completely as can be seen from the electron microscopy pictures in figure 36. The result is less dense muscle and with completely different density distributions, which is not at all homogenous; large areas having low density alternates with others having high density as can be seen in figure $47, \mathrm{C}$. This morphological characteristic can be explored for modelling the muscle fibre direction.

The general idea is to track the high dense areas on the muscle cross sections in order to create a path along the muscle which passes through the denser muscle zone. Of course on every cross section there will be many locations with this characteristic therefore many paths will be created following the main muscle fiber direction. Theoretically if such algorithm would be very accurate and the tissue discrimination not suffering from partial volume effects then the density tracker could actually display the muscle fiber direction. In reality the pixel size is too big to discriminate between muscle and other surrounding tissue and therefore such approach is of difficult implementation on healthy musculature. But 


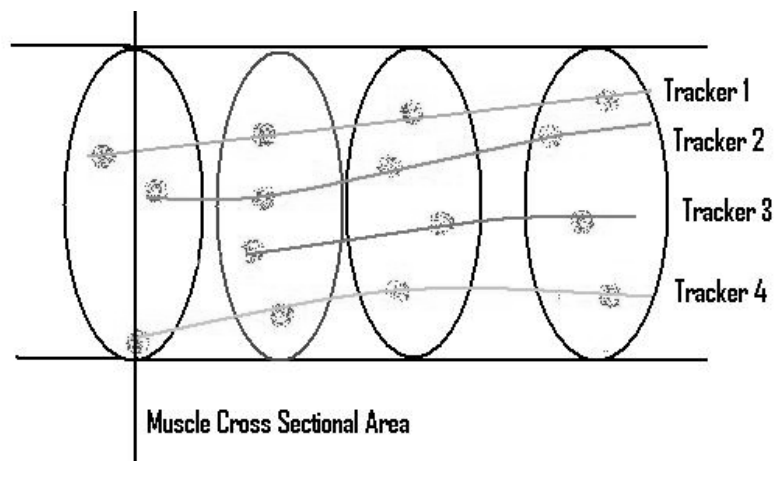

Fig 48 Schematic representation of density tracker

in denervated muscle where the density is highly inhomogeneous (Fig. 47, C) an approach like that could make sense and being more feasible.

In fact, in denervated and degenerated muscles the areas with high density are not contiguous and are more discretely distributed. Therefore such density trackers would be displaying the main patterns where the density is higher along the muscle and giving in this way an indication on residual muscle fiber length and direction. The process is represented in figure 48 in a simplified manner

\subsubsection{Implementation: central line method}

The method used to implement this approach is based on sub thresholding and central line distribution. The central line method was used in the geometrical analysis to model the shape changes in rectus femoris (paragraph 4.5.1). The method is adapted in this framework to allow central lines to model muscle direction. The central line is defined as the interpolation of central points through the cross sectional area of a 3-Dimensional object. The central points in each cross section are unique or multiple depending from the number of edges and from the object density. To allow central lines to model fiber direction the muscle threshold must be reduced drastically in order to visualize only full dense muscle tissues. For this reason the new threshold interval is set on 60-90 HU. The consequence on the denervated

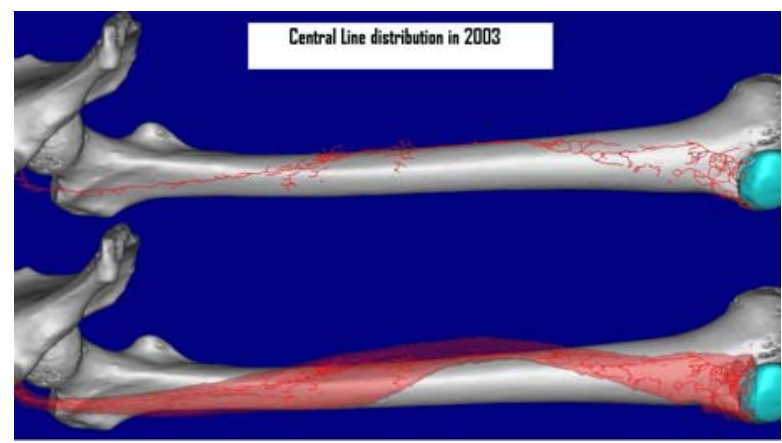

Fig 50 Central line distribution in 2003, the muscle is denervated and not stimulated.

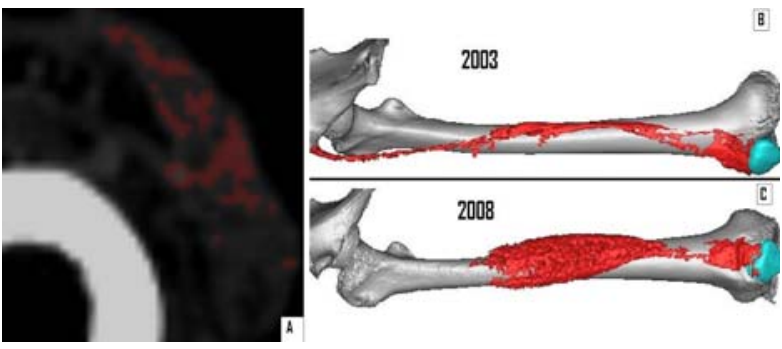

Fig 49 Rectus femoris with threshold interval of 60-90 HU: Cross sectional area 2003 (A), 3Dimensional Model 2003 (B), 3-Dimensional Model 2008 (C).

rectus femoris cross section is seen in figure 49 , A. The $3-\mathrm{D}$ models rendered with the reduced threshold are also looking differently from those showed in figure 42. The new 3-D models in figure 49, B and $\mathrm{C}$ shows rectus femoris from patient 2 with the new threshold in 2003 and in 2008.

With the new set up the central line will be different from the one calculated in paragraph 4.5.1 because of the new distribution of voxels within 3-Dimensional object. Basically, we have now established the environmental condition described in figure 48 and we will use central lines as "density tracker".

The central line algorithm (developed in MIMICS software [44]) is applied on both 3-D models (Fig. 49, $\mathrm{B}, \mathrm{C})$ and the resulting central line distribution displayed in figure 50 and 51.

The central line distribution from 2003 (Fig. 50) has a main central path and many small branches which doesn't seem to be organised and having any definite direction.

The central line distribution from 2008 (Fig. 51) has a main central path too, but the numerous branches aren't randomly distributed like in the previous case. Indeed these branches are longer and converge to the centre recalling the bipennate architecture characteristic of rectus femoris.

Concluding the modelling technique used must be validated and developed further but this preliminary

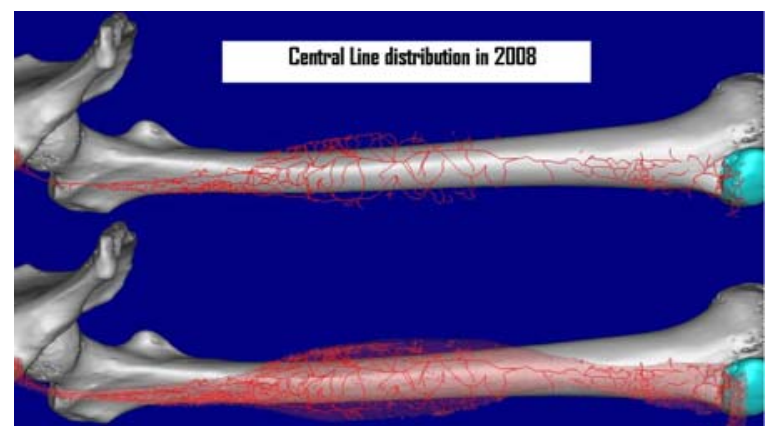

Fig 51 Central line distribution in 2008, the muscle is denervated and stimulated for 4 years. 

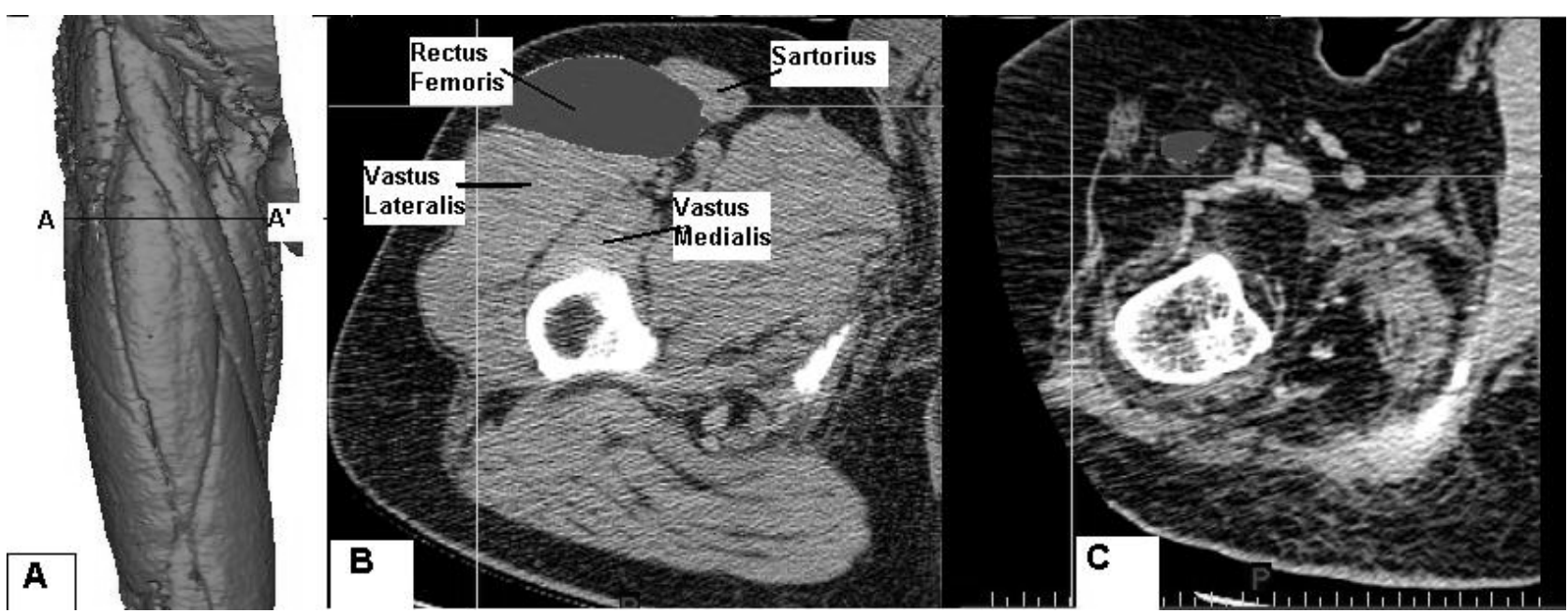

Fig 52 (A) Healthy right leg, the A-A' segment display the height where the cross sections are compared: (B) Healthy subject, (C) 4-years denervated leg

work shows the remarkable differences in fiber direction and architecture between denervated muscles pre and post stimulation treatment.

\section{Modelling and Analysis of Quadriceps Muscle 5.1 Introduction}

The core modelling work has been developed in the previous chapter around the analysis of rectus femoris muscle. One reason is because the segmentation techniques are easier implemented on this muscle, since it can be isolated from the surrounding even after long term degeneration. Another reason is that the monitoring of rectus femoris growth is very indicative to estimate the treatment efficacy; indeed the muscle is positioned in the middle of the quadriceps musculature and therefore particularly exposed to the electrical stimulation. Nevertheless all quadriceps muscles are targeted by the stimulation treatment and in order to validate and improve the clinical trial monitoring should involve these bellies as well. In this chapter the segmentation techniques developed and used to study rectus femoris are adapted and applied to monitor changes in the other quadriceps muscles.

Changes in muscle and fat volume in the thigh will also be measured in order to study correlations between muscle growth and fat loss and vice versa.

\subsection{Segmentation of quadriceps muscle}
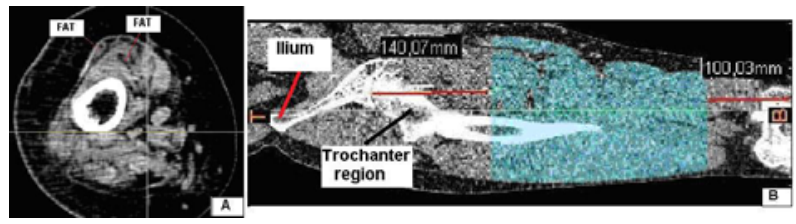

Fig 53 (A) Fat tissues in the region of vastus lateralis. (B) Region of interest start $140 \mathrm{~mm}$ below from the femur head and ends $100 \mathrm{~mm}$ above the patella base
The stimulation effectiveness is in general sensible to a number of factors such as patient compliance, regime of stimulation and degree of muscle degeneration. Beside there other issues influencing the local distribution of the stimulus along the muscles, such factors are for example surface electrode position and tissue composition on the target region. Therefore there are many parameters that can improve or diminish the stimulation effect. To measure the muscles changes selectively and study the factors influencing the muscle grow, the muscle bellies in the quadriceps are segmented and volume and density changes monitored. The processing problems related to the segmentation of denervated muscles were previously discussed in paragraph 4.1. The extreme degeneration occurring in quadriceps muscles is the main issue to be addressed using the segmentation approach. In fact in vastus lateralis, medialis and intermedius, the cross-sectional area became extremely thin cause long term denervation, and consequently these muscles are almost unrecognizable on the CT scan images. This is particularly true and evident in proximity of the muscle bellies ends; in those regions where the muscles are attached to the bone. In fact rectus femoris is the longest quadriceps muscle, it originates on the ilium while the other three muscles originate from the body of the femur, which they cover from the trochanters to the condyles. All four parts of the quadriceps muscle attach to the patella (knee cap) via the quadriceps tendon (Fig. 53, B).

In figure 52 are compared two thighs cross sectional areas in proximity of vastus medialis and lateralis ends (A-A' segment in figure 52, B) in normal subject and in paraplegic after 4 years denervated muscles. The muscles cross sectional area in figure 52, C show how quadriceps bellies are reduced after long term denervation and becoming not longer recognisable. This dramatic muscle deterioration is of course a limit 


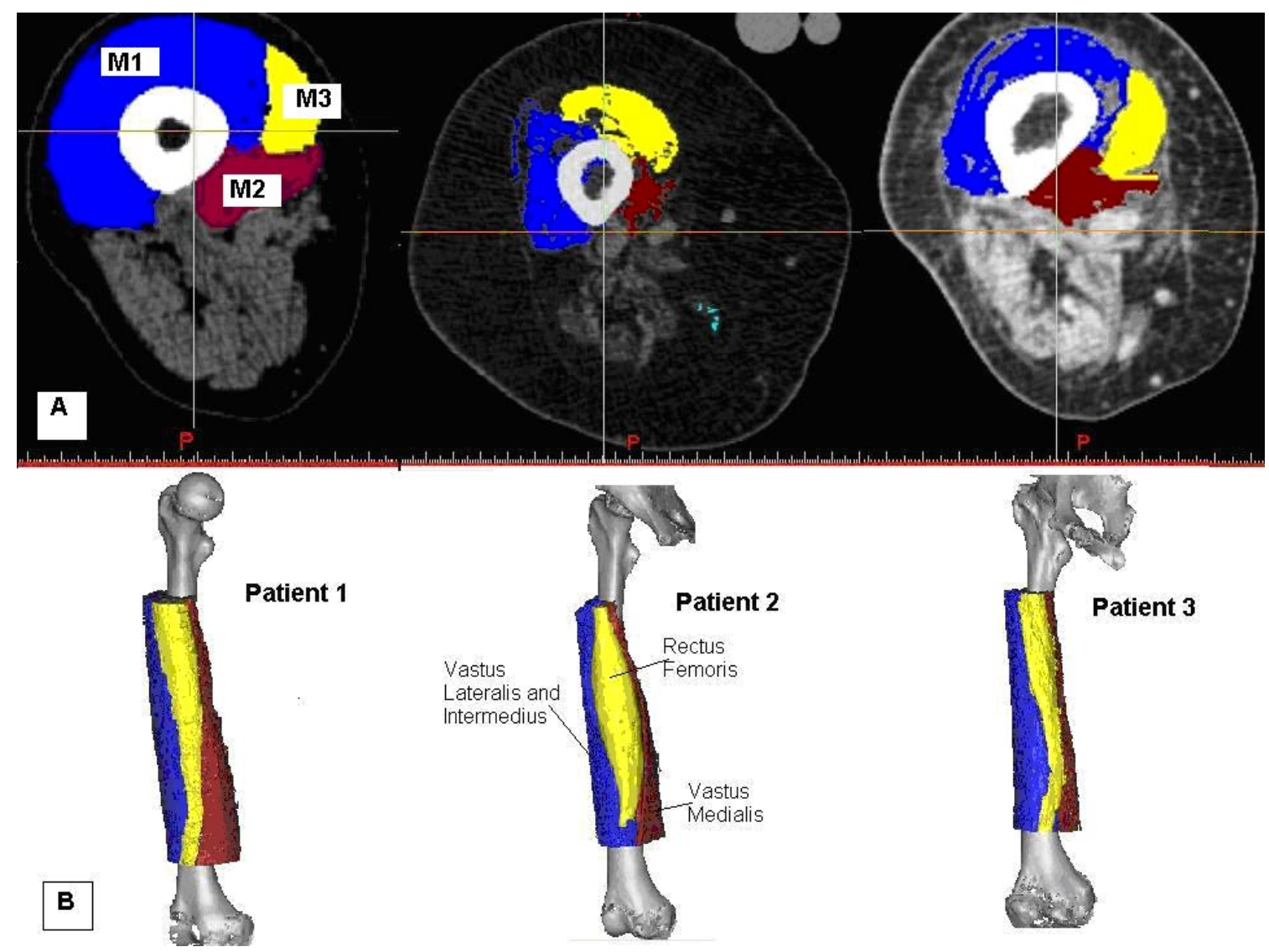

Fig 54 (A) Segmentation masks on the cross sectional area. (B) 3-Dimensional models of the segmentation masks

for the segmentation. In this regions isn't possible to be precise and therefore a compromise solution was found; the muscle in quadriceps are segmented in a region of interest which cover the majority of the muscle volume but not all of it. In fact the most degenerated parts are excluded from the segmentation process. The segmentation process is applied in a defined region of interest which starts $140 \mathrm{~mm}$ below from the femur head and ends $100 \mathrm{~mm}$ above the patella base (Fig. 54, B). The threshold interval chosen for the quadriceps segmentation is $[-25,135] \mathrm{HU}$; the threshold is wider respect the one used in the rectus femoris segmentation (in paragraph 4.2 it is used the threshold -5, 135). The reason for enlarging the threshold is to allow a proper display and measurement for the abundant fatty tissue present particularly in the region of vastus lateralis (Fig 52.A). The segmentation results and quadriceps muscle belly map are displayed in figure 54. The developed segmentation masks are three: M1, M2 and M3 representing respectively, vastus intermedius and vastus lateralis together, vastus medialis and rectus femoris. The reason to combine in the same mask vastus lateralis and intermedius is because the edges of vastus intermedius disappear completely during the degeneration process making segmentation impossible.
Finally the segmentation process described in chapter 4 produces three segmentation masks which can be 3dimensional modelled and reconstructed as displayed in figure $54, \mathrm{~B}$.

\subsection{Measurement of volume and density}

3-Dimensional modelling and visualization is an important outcome from segmentation work which can be used to study morphological changes of the muscle as demonstrated in the analysis of rectus femoris shape and the geometrical changes in chapter 4.5. These studies are not repeated on the quadriceps muscle since the muscles volume are not entirely represented and therefore such analysis would not make much sense. Instead the segmentation masks are used to access information otherwise hidden such as changes in volume and density as consequences of FES treatment. Indeed these data are immediately available from the segmentation mask.

\subsubsection{Measurement of volume}

The quadriceps muscle are segmented since the beginning of the clinical trial in 2003, gray value interpolation algorithm (paragraph 4.3.1) is used to calculate and measure the segmentation mask volume, the results are displayed with histograms in figure 55. 


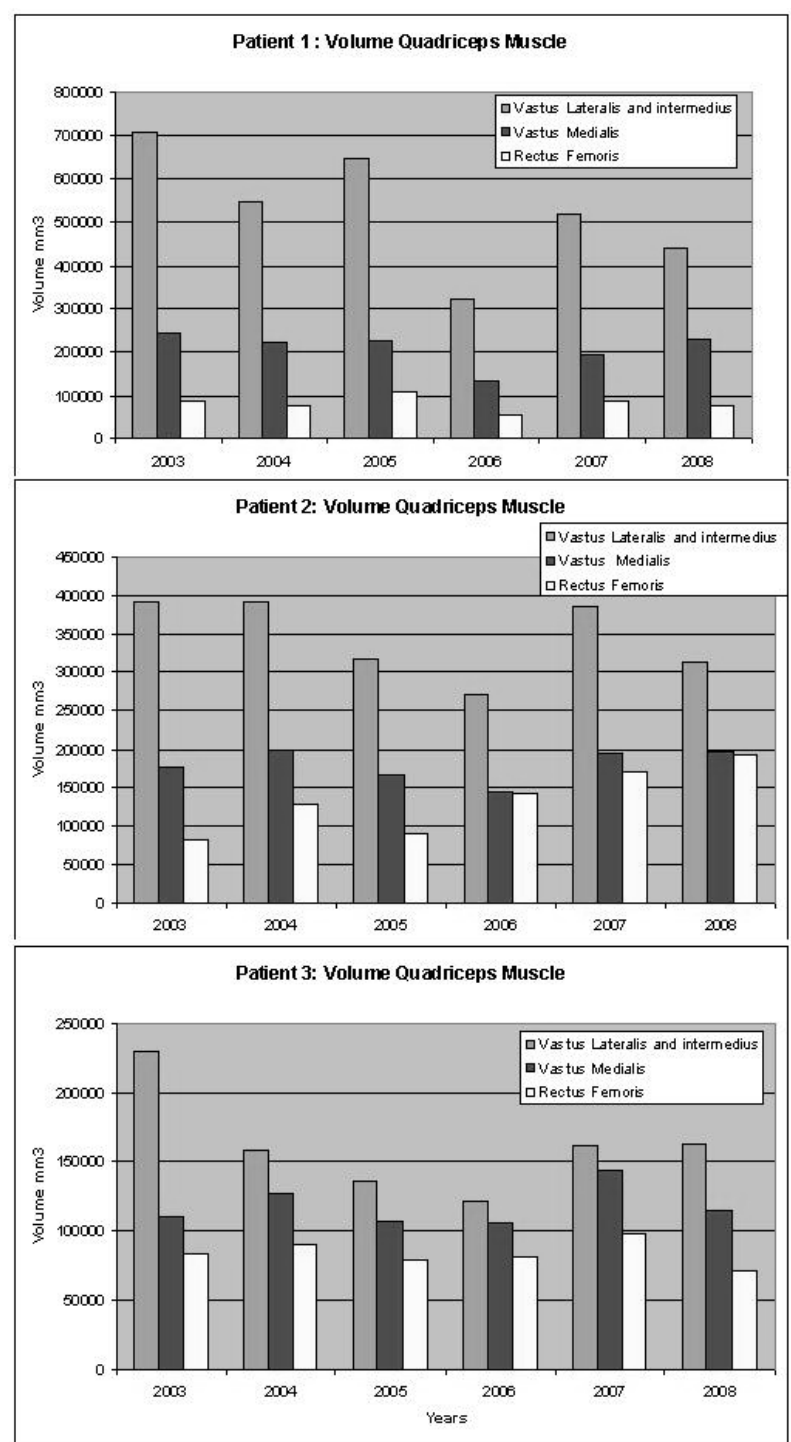

Fig 55 Volume measurement in the quadriceps (inside the ROI): 1st histogram patient 1, 2nd histogram patient 2 and 3 rd histogram patient 3

The histogram bars show the different muscle belly volume in the region of interest during the clinical trial. In patient 1, vastus lateralis and intermedius appear as the quadriceps muscle more sensitive to stimulation and as well to degeneration. Indeed for these bellies the volumes vary considerably during the monitored period; for instance in 2006 the muscles are halved compared to the previous year. The changes in other bellies are smoother but basically in line with the behaviour in vastus lateralis and intermedius. In fact rectus femoris and vastus lateralis in patient 1 are not changing much during the monitored years except in 2006 where loss of volume is remarkable caused by interruption of the stimulation treatment.

In patient 2 the variation between the muscle bellies are rather contradictorily. Indeed while rectus femoris grow remarkably and continuously since 2005 vastus lateralis and intermedius vary as well but not in line with it; the volume in this segmentation mask is decreasing in 2006 increasing remarkably in 2007 and then decreasing again in 2008. The changes in vastus medialis are instead smoother and following rectus femoris behaviour.

The volumes measurement from patient 3 show a dramatic drop in vastus lateralis and intermedius in 2004 though isn't muscle degeneration but a surgical removal to care a severe pressure sore on the patient gluteus. Due good patient compliance, in 2007 the volume grows substantially in all the bellies but then the stimulation treatment is interrupted in late 2007 because of new clinical problems and the muscle volume drops again in 2008 .

The results from the volume measurement underline the different sensitivity and response of quadriceps muscle toward stimulation:

- The volume increases on certain bellies while it reduces or is unchanged in other during the same period. In patient 2, for example, between 2007- 2008 rectus femoris and vastus medialis increase while vastus lateralis and intermedius reduce. In this case the electrical stimulus doesn't reach the all quadriceps homogenously and effectively probably because of the very thick layer of fat surrounding the muscle (quantitative measure in table 5.2).

- Volume increases / decreases more in certain bellies than in other. Such occurrence is seen between years 2006-2007 in patient 1, here vastus lateralis and intermedius increase a lot more compared to increase measured in the other bellies. This probably depends both from the electrodes positioning and from the quadriceps morphology which in the case of patient1 is characterised by an extended area occupied by vastus lateralis (cross sectional area in figure 54, A).

\subsubsection{Measurement of density distribution}

The segmentation masks display HU values included in the interval $[-25,135]$, the average HU value over the pixels contained in the mask is calculated and the results are gathered in table 8 . These data account to the muscle density in the denervated quadriceps. A comparison of these values allows evaluating the degree of restoration among the quadriceps muscles. In normal subject the mean $\mathrm{HU}$ value in the quadriceps is between 55-65 HU.

In patient 1 the starting density values are high because of the low level of denervation in the muscles. In all bellies mean muscle density increase when the patient is compliant though the mean density value beat higher values in vastus lateralis and intermedius. The peak values are registered in 2007 , in line with volume increases (Fig. 53).

In patient 2 the density values at beginning (2003) are particularly low in vastus medialis and intermedius and vastus medialis. These bellies obviously aren't properly stimulated since their density decrease while 
3D Modelling and monitoring of denervated muscle under FES

\begin{tabular}{|c|c|c|c|c|c|c|c|c|c|}
\hline & \multicolumn{3}{|c|}{ Patient 1} & \multicolumn{3}{|c|}{ Patient 2} & \multicolumn{3}{|c|}{ Patient 3} \\
\hline $\begin{array}{c}\text { Years/HU } \\
\text { Avg. }\end{array}$ & $\begin{array}{c}\text { Vastus } \\
\text { Lateralis }+ \\
\text { lntermedius }\end{array}$ & $\begin{array}{c}\text { Vastus } \\
\text { Medialis }\end{array}$ & $\begin{array}{l}\text { Rectus } \\
\text { Femoris }\end{array}$ & $\begin{array}{c}\text { Vastus } \\
\text { Lateralis }+ \\
\text { Intermedius }\end{array}$ & $\begin{array}{c}\text { Vastus } \\
\text { Medialis }\end{array}$ & $\begin{array}{l}\text { Rectus } \\
\text { Femoris }\end{array}$ & $\begin{array}{c}\text { Vastus } \\
\text { Lateralis }+ \\
\text { lntermedius }\end{array}$ & $\begin{array}{c}\text { Vastus } \\
\text { Medialis }\end{array}$ & $\begin{array}{l}\text { Rectus } \\
\text { Femoris }\end{array}$ \\
\hline 2003 & 42,7 & 35,7 & 44,9 & 22,3 & 17,7 & 33,7 & 29,3 & 28,6 & 34,7 \\
\hline 2004 & 42,1 & 33,8 & 47,6 & 23,2 & 27,9 & 35,5 & 31,6 & 33,4 & 39,4 \\
\hline 2005 & 47,1 & 43,9 & 43,5 & 17,6 & 19,7 & 38,3 & 27,3 & 31,6 & 36,2 \\
\hline 2006 & 43,6 & 40,5 & 41,4 & 23,9 & 29,5 & 41,2 & 25,2 & 30,5 & 37,9 \\
\hline 2007 & 48 & 48 & 44,2 & 17,1 & 22,9 & 37,2 & 23,9 & 29,2 & 36,4 \\
\hline 2008 & 42,9 & 40,3 & 42,8 & 17,4 & 26,7 & 40,6 & 19,4 & 19,5 & 26,7 \\
\hline
\end{tabular}

Table 8 Mean density values in the quadriceps muscles during the clinical trial

in rectus femoris is increasing (2004- 2005). The peak density values are reached in 2006.

In patient 3 the density have a peak in 2004 otherwise the average values are actually not changing much, except in 2008 where due extended edema in lower limbs the patient muscle loses density dramatically. In general the data in table 8 suggest a better responding to stimulation in rectus femoris compared to other muscle in the quadriceps. Indeed, in all patients at least ones during the FES trial was measured simultaneous increase in rectus femoris density and decrease in other quadriceps bellies. This fact occur in patient 1 in the years 2003-2004, in patient 2 in 2004-2005 and finally in patient 3 in 20052006.

Concluding, rectus femoris density is in general higher compared to other muscle in the quadriceps both at beginning and then during the electrical stimulation trial: it is degenerating "less" respect other bellies meaning that infiltration of fat and connective tissues within the muscle fiber is lower. Another explanation to justify the higher muscle density in rectus femoris can be found in the muscle morphology and specifically in the robust tendons structure present in the muscle. Indeed rectus femoris arises by two tendons, the two unite at an acute angle, and spread into an aponeurosis which is prolonged downward on the anterior surface of the muscle, and from this the muscular fibers arise. The muscle ends in a broad and thick aponeurosis which occupies the lower two-thirds of its posterior surface, and, gradually becoming narrowed into a flattened tendon, is inserted into the base of the patella. When the muscle degenerate and lose density, the mean HU value remain still higher compared to other quadriceps muscle because of the large tendons tissues present in the muscle which density is between 70-80 HU as seen in figure 56 .

\subsubsection{Muscle tissue structure analysis}

The profile line method developed in paragraph 4.4.1 is used to display and compare the tissue composition in the quadriceps muscle. Three profile lines within respectively vastus lateralis and intermedius, vastus medialis and rectus femoris are drawn along the coronal plane. The density values intercepted by the profile lines are coded with three values:

- 1: HU >= 55 (High muscle density)

- $0: 10=<\mathrm{HU}<55$ (Medium muscle density)

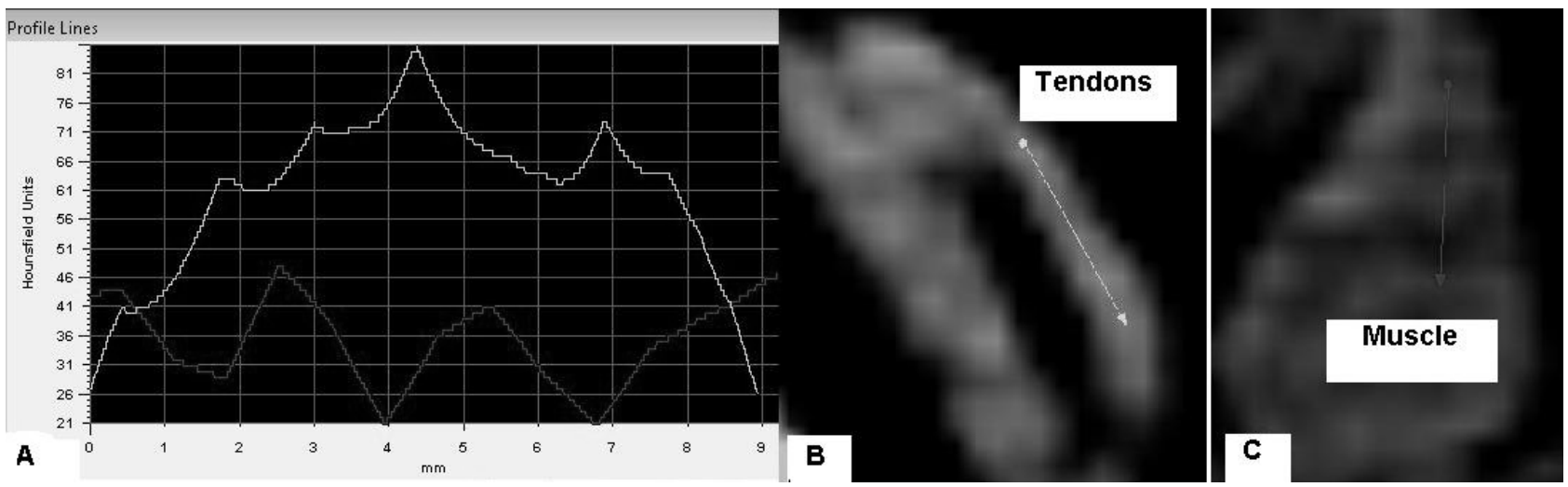

Fig 56 Comparison between density in tendons and muscle in a 7 years denervated rectus femoris (A): Profile line in the tendons area in proximity of the patella $(B)$ and profile line in the middle of the muscle $(B)$. 
Patient 1
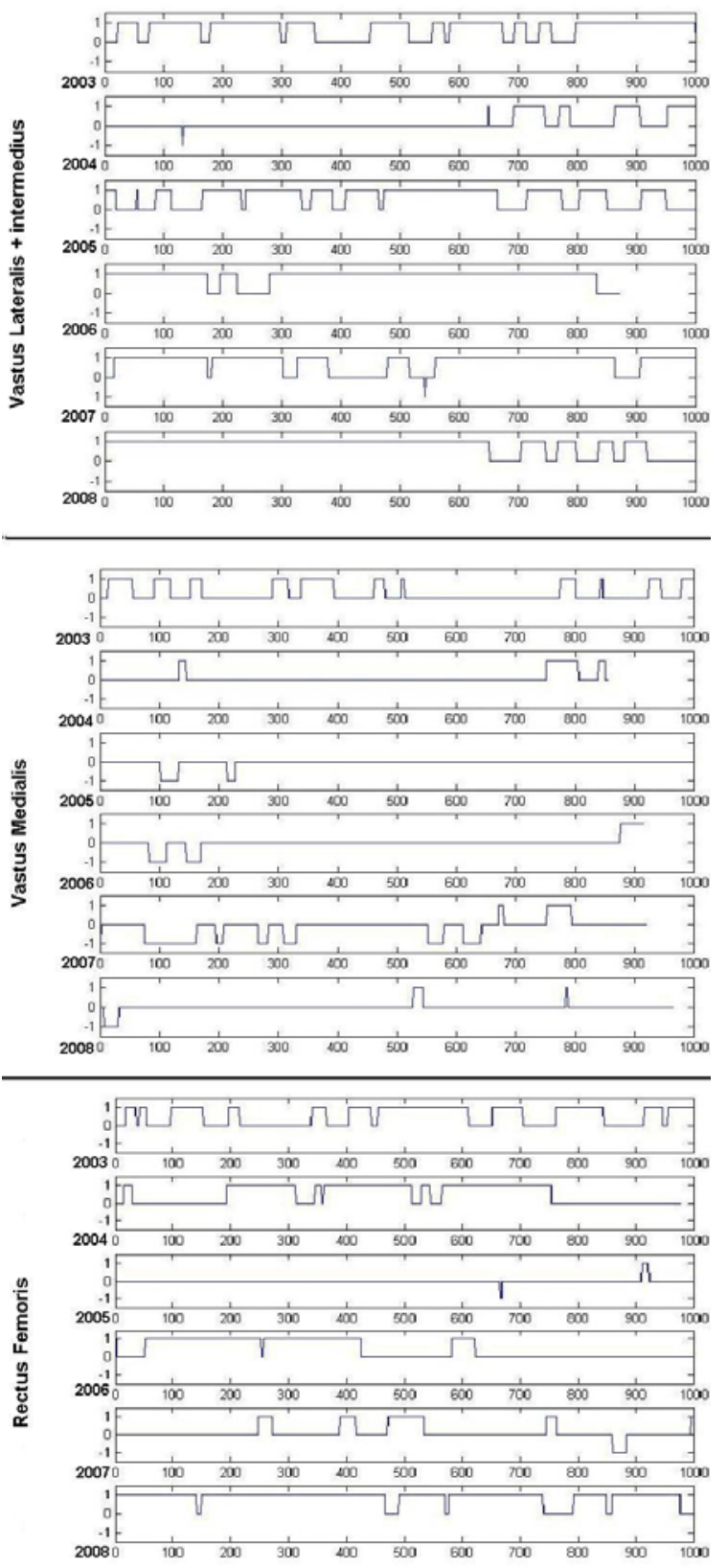

Fig 57 Profile line distribution in the quadriceps muscle for patient 1: 1st panel vastus laterals and intermedius, 2nd panel vastus medialis and $3 r d$ panel rectus femoris

$--1: \mathrm{HU}<10$ (Low muscle density)

The profile line analysis is a great tool to look close into the muscle fiber but it is limited to the segment of muscle intersected by the line which in our case is 100 $\mathrm{mm}$. Therefore the results maybe discord from the mean density values found in table 5.1 which instead account the whole segmentation volume. On the other
Patient 2
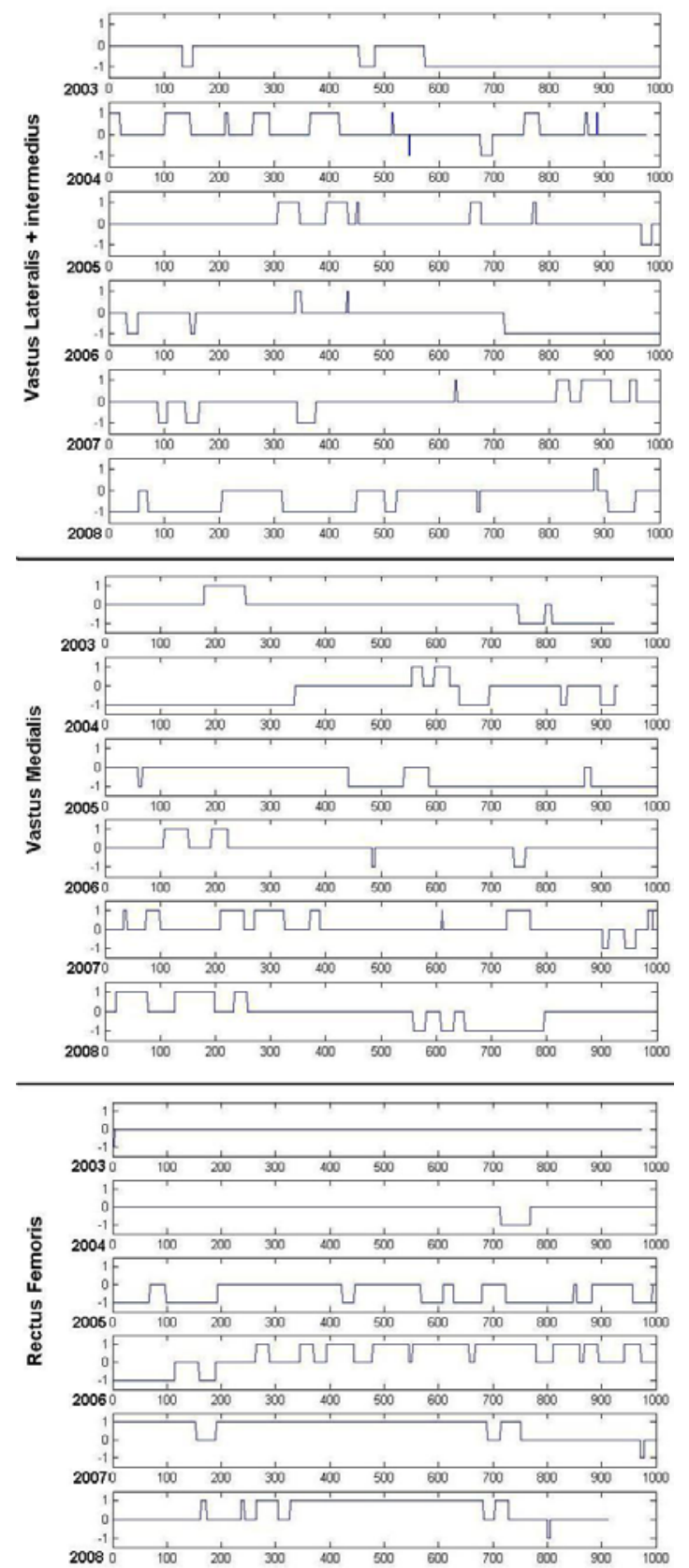

Fig 58 Profile line distribution in the quadriceps muscle for patient 2: 1st panel vastus laterals and intermedius, 2nd panel vastus medialis and $3 r d$ panel rectus femoris

end the profile line methodology enable to measure selectively the tissue composition in defined regions and through established directions.

In normal muscle the profile line as showed in paragraph 4.4.2 figure 4.13, is basically coded with 1 and spaced with 0 values in restricted segments. Profile lines from denervated muscle have in general more and 

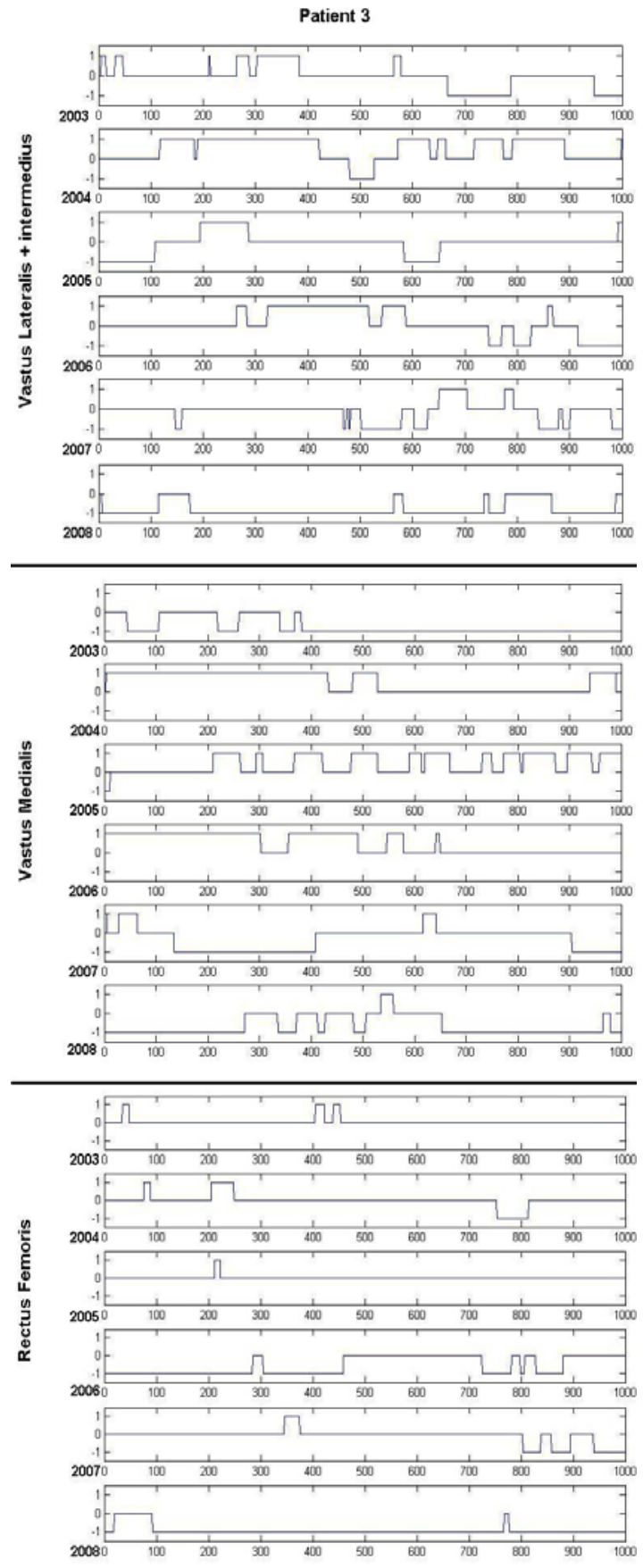

Fig 59 Profile line distribution in the quadriceps muscle for patient 3: 1st panel vastus laterals and intermedius, 2nd panel vastus medialis and $3 r$ d panel rectus femoris

larger area coded with 0 and when the muscle is severely degenerated, like in the case of patient 3 , the density profiles display only 0 and -1 values.

The results from the profile lines analyses in quadriceps are displayed in the Figs 57-59. This kind of analysis combined with the mean density values in

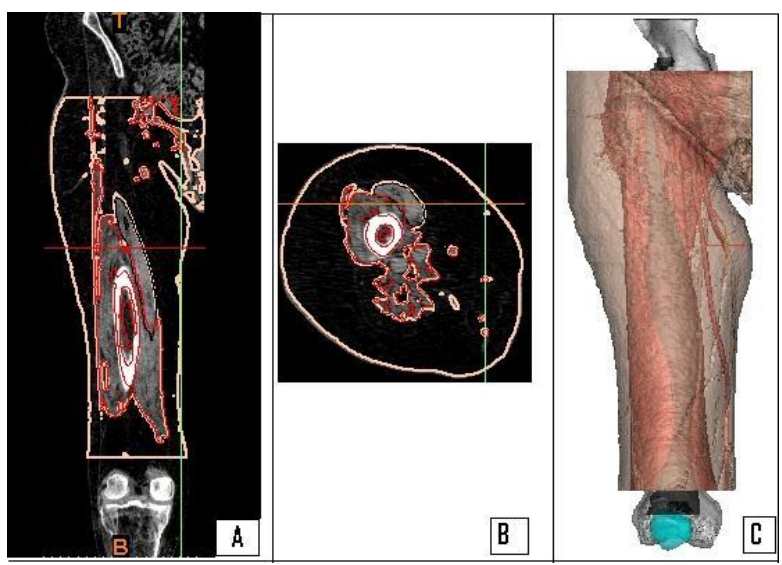

Fig 60 Segmentation region which starts below the femur head and end above the patella. (A) sagittal plane, (B) axial plane and (C) 3-D rendering of fat and muscle in the region of interest

table 8 give a more complete picture of the changes in the muscle tissues, some remarks follow:

- In accordance with the mean values gathered in table 8 , the tissue composition in patient 1 vastus lateralis and intermedius appear dense and compact with no areas coded with -1 and short sections coded with 0 . In patient 2 is again showed the scarce impact of FES treatment on vastus lateralis, intermedius and medialis.

- In patient 3 the main remarks are the clear improvement in muscle tissue composition seen in vastus lateralis in 2004 and again in 2006 which demonstrate how restoration can occur also in such long denervated musculature.

\subsection{Monitoring of muscle and fat}

Increase of fat in the thigh and within the muscle is a normal consequence of muscle idleness; of course such fact is particularly developed in denervated musculature where the inactivity is complete. The ability of denervated muscle to contract again with the help of external electrical stimulation was demonstrated and even a muscle growth which follow the stimulation treatment. It is then interesting to measure the effects and the correlations between the induced muscles grow and changes in fat volume.

The patient's right legs are monitored and muscle and fat changes measured in defined regions of interest which starts below the femur head and end above the patella (figure 60, A). The muscle and fat tissue are displayed and measured using segmentation masks created using the following Hounsfield intervals: [-5, 135] HU for the muscle and the [-205, -6] for the fat. The two intervals do not overlap each other and 


\begin{tabular}{|c||c|c|c|c|c|c||}
\hline $\begin{array}{c}\text { Volume } \\
\text { (mm3) }\end{array}$ & Pt.1 Muscle & Pt.1 Fat & Pt.2 Muscle & Pt.2 Fat & Pt.3 Muscle & Pt.3 Fat \\
\hline \hline $\mathbf{2 0 0 3}$ & 1655103 & 141000 & 931619 & 463000 & 961290 & 247000 \\
\hline $\mathbf{2 0 0 4}$ & 1352534 & 141000 & 922440 & 479000 & 671634 & 223000 \\
\hline $\mathbf{2 0 0 5}$ & 1679577 & 142000 & 759600 & 513000 & 608786 & 301000 \\
\hline $\mathbf{2 0 0 6}$ & 1391829 & 108000 & 802981 & 448000 & 802909 & 354000 \\
\hline $\mathbf{2 0 0 7}$ & 1632142 & 112000 & 890037 & 498000 & 925668 & 364000 \\
\hline $\mathbf{2 0 0 8}$ & 1553218 & 121000 & 920375 & 478000 & 1008712 & 496000 \\
\hline
\end{tabular}

Table 9 Values of muscle and fat volume

therefore no further processing work is required to separate the tissues and to create the masks.

Muscle and fat volume are directly measured from the segmentation masks (as sum of voxels) and the results. It is immediately noticeable that the amount of fat in patient 2 is greater compared to the other two patients, this probably explain the poor response in the lateral quadriceps muscle to FES. Anyway table 9 shows that changes in fat during the monitored period are in same order as in muscles, suggesting that the fat contained in the region of study is influenced somehow from the stimulation treatment. To study the possible correlation between muscle and fat variation the values in table 9 are displayed in figure 61 as percentage of their variation in respect the initial data in 2003. In this way increases or decreases in fat and muscle volume can be estimated and correlated.

The changes in muscle volume appear smoother compared to the histograms in figure 55. This because

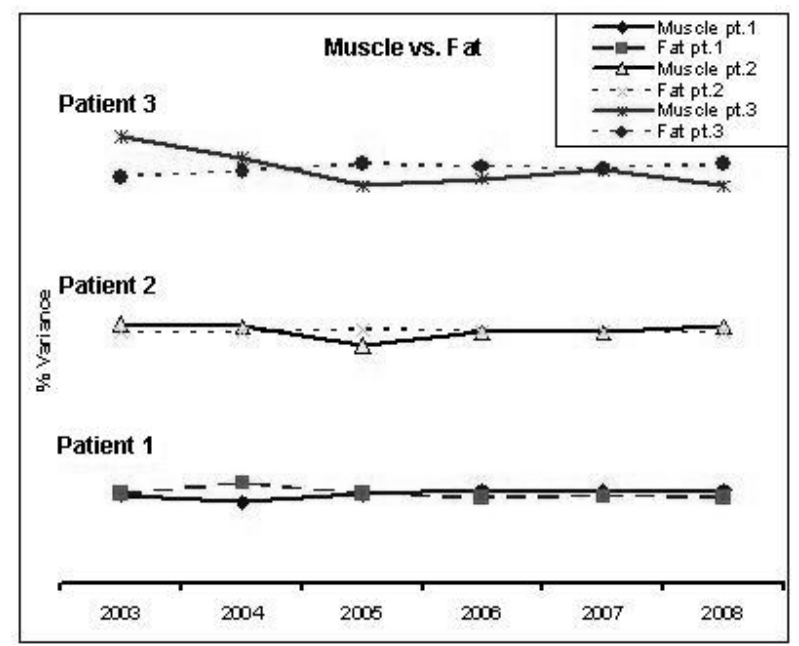

Fig 61 Volume variability (\%) between muscle and fat during the clinical trial the volume measured includes all the muscle in the thigh and therefore also those which are marginally or not at all stimulated.

The trend line in figure 61 shows a certain correlation between decrease of fat and increase of muscle. Indeed the variation of fat and muscle appear inversely proportional. This fact is particularly evident in patient 3 during all the clinical trial, but also in patient 2 in years 2005 and 2008 and in patient 1 in years 2004 and 2006.

\section{Bone Structural Changes and Mineral Density Distribution in the Patella \\ 6.1 Introduction}

Shortly after the spinal cord injury the bones of the paralysed extremities incur in atrophy and mineral loss. Osteoporosis is one of the major complications in patients with SCI, indeed in paraplegic patients immobilization induces severe bone loss becoming the bone more and more fragile with dramatic increasing of fracture risks (Fig. 6.1). SCI causes immediate and permanent gravitational unloading. The result is a disuse structural change with associated metabolic consequences. Degeneration and reaching of fracture threshold occur at one to five years after injury [48]. Both longitudinal $[49,50]$ and cross-sectional $[51,52$ and 53] studies have documented extent and timing of this bone atrophy.

The bone resorption process begins to occur within days after SCI; osteoclasts break down bone and release the minerals, resulting in a transfer of calcium from bone fluid to the blood. The body starts dumping minerals such as calcium into the urine. Although sensible bone loss can be seen on x-rays only after several months from the SCI, calcium and other chemicals released during bone breakdown can be measured shortly after the injury in blood and urine test [55]. This bone loss is greatest in the first 3-12 months after injury, but still persists for several years after 


\section{D Modelling and monitoring of denervated muscle under FES}

European Journal Translational Myology - Basic Applied Myology 2011; 21: 31-94

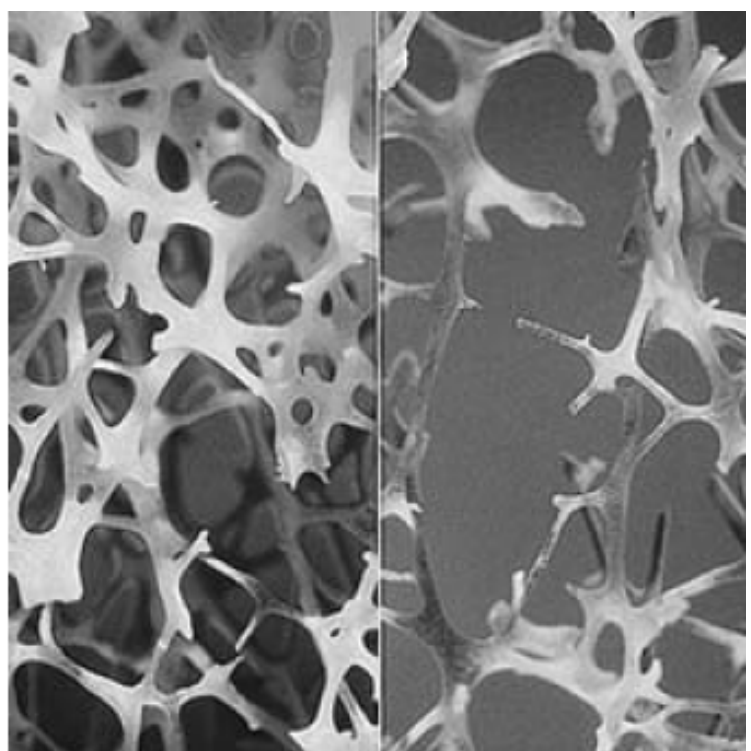

Fig 62 Comparison of trabecular bone in a healthy person (on the left) and in one who is suffering from osteoporosis [54]

your injury [56]. The areas rich in trabecular bone are demineralized to the greatest degree; distal femur and proximal tibia are the bones most affected, followed by the pelvis and arms [57].

Szollar et al. [48] measured BMD of the lumbar spine and three regions of the proximal femur in 175 SCI men and 62 controls without SCI. The results exhibited a progressive degradation in BMD at the proximal femur. Participants were divided into two groups according to age. The bone mass reached fracture threshold in the 20 to 39 years age group 1 to 9 years after injury. In the 30 to 49 year age group the degradation also began 1 to 9 years after injury but progressed at a slower rate and reaching fracture threshold at 10 to 19 years after injury. The spine BMD remained stable above fracture threshold in both age groups. Fractures in this patient population most commonly occurred at the proximal femur and knee.

The mineral loss in SCI subject is more acute in respect osteoporosis caused by other reasons such as prolonged bed rest or other neurologic deficits. The explanations for such dramatic break down in bones after spinal cord injury are not completely clear. One factor is of course inactivity and the loss of muscles traction and load on the bones. But other factors such as changes in the autonomic nervous system and/or circulatory system are probably playing a role in the resorption process occurring after [58].

In general treatment for osteoporosis focuses on slowing down or stopping the mineral loss, increasing bone density, preventing bone fractures, and controlling the pain associated with the disease. A number of medications are available for this purpose, such as alendronate, risedronate, raloxifene, ibandronate, calcitonin, and zoledronate.

Alternative to the medications other treatment like electrical stimulation has been showing beneficial effects on bone strengthen. Indeed electrical stimulation for bone repair has been used for three decades to treat inadequate healing of the fracture (pseudarthrosis) and bone non-union [59]. Mechanical or electrical stimuli of relative low amplitude and high frequency can influence bone formation and resorption in vitro and in vivo, suggesting that these modalities can be used clinically to inhibit or reverse loss in bone density.

The treatment of bone loos in SCI subject using electrical stimulation has also been largely investigated [59, 60-62].

Animal experiment utilizing a sciatic neurectomy model, demonstrates increases in bone formation using capacitively coupled electrical signal [63].

Other studies demonstrate that electrically induced muscular contraction reduced bone mineral loss in the paralysed limb during early stage of SCI in the rabbit [64].

In human many studies have study and assessed the influence on FES-induced cycling. Several studies found no remarkable difference in BMD of the lower limbs between before and after the FES-cycling intervention while other study suggests a reduced rate of SCI-induced bone loss and therefore a potential beneficial effect [64-69].

However there is still a conflicting evidence and not convincing trends about the efficacy of electrically evoked muscle activation in the stimulation of bone metabolism (bone formation and/or reduction of bone loss), and the clinical role of functional electrical stimulation (FES) in the treatment of osteoporosis resulting from neurological damage [70].

The influence on the bone density due FES treatment on the denervated quadriceps muscle has been studied as secondary effect in the Icelandic site of the RISE project. In this chapter bone structural changes are analysed using 3-D modelling and bone mineral indexes. Special attention is paid in study those changes in the patella bone.

\subsection{Changes of bone density in femur bone as consequences of FES treatment on the quadriceps muscle}

The bone density changes in SCI patients treated with FES has been studied in Iceland since year 2004 with the aim of demonstrates correlation between bone density changes and electrical stimulation treatment [71-73].

The following text is an extract from the published article: Using Mimics to Monitor Changes in Bone Mineral Density of Femur during Electrical 


\section{D Modelling and monitoring of denervated muscle under FES}

European Journal Translational Myology - Basic Applied Myology 2011; 21: 31-94

Stimulation Therapy of Denervated Degenerated Thigh Muscles. T. Helgason, P. Gargiulo, P. Ingvarsson, S. Yngvason.

This paper has participated and won the MIMICS Innovation Award (2007), in Washington (USA).

This study proposes a method to measure bone density and structural changes in femoral bone using Helical CT Scans and 3-Dimensional modeling. The patients groups are paraplegic patients with complete denervation in the lower limbs undergoing FES treatment.

The femur bone tissue is segmented according to six Hounsfield unit (HU) intervals. This gives six models, each for one density interval and therefore of a part of the femur. Shape, structure and statistics of the models for each scanning are compared using the Mimics tools.

Results show that the total amount of bone tissue is not changing, that remodeling of the bone takes place with an increase in the densest part of the bone at locations where force is expected from the stimulated muscle but a decrease at other location. This suggests that the electrical stimulation therapy is contributing to remodeling of the femur bone.

Each spiral CT scan gives one data set showing the tissue situation at the time scanning. From every data set the femur bones are segmented using the Mimics software from Materialise [44]. Then a region of interest (ROI) was chosen as the area between a line $140 \mathrm{~mm}$ below the femur head and $100 \mathrm{~mm}$ above the knee joint. Figure 63, A shows the position of the ROI. The ROI is then processed. This area is segmented according to six Houndsfield unit intervals, that is: $150-350 \mathrm{HU}, 351-550 \mathrm{HU}, 551-850$ HU, $851-1150 \mathrm{HU}, 1151-1350 \mathrm{HU}$ and 1351 - $1536 \mathrm{HU}$. This segmentation corresponds to going from the inside of the bone, with $150 \mathrm{HU}$ for the trabecular bone with the lowest density, to the outside of the bone, with $1536 \mathrm{HU}$ for the cortical bone with the highest density. Each HU interval gives roughly a cylindrical shape and they are concentric.

Examples of the results are shown in figure 63, B and $63, \mathrm{C}$. As can be seen in the second cylinder from inside (colored in green) it is full of holes. This is due to the thickness of the cylinder and direction of sampling matrix of the CT. Since the thickness is in the range of the voxel size the voxels have to land to a great extent inside the area with a density of $351-550$ $\mathrm{HU}$ to get a corresponding $\mathrm{HU}$ value. Otherwise they get an HU value above or below this interval. And since the direction of the sampling matrix of the CT scan is tilted with a small angel to the axis of the cylinder areas of voxels get a HU value inside the 351 - $550 \mathrm{HU}$ interval and other areas are outside this interval. This gives the shape see in figure $63, \mathrm{~B}$ and 63, C.

Where a plane in the sampling matrix crosses the cylinder the voxels are green and where the cylinder is between planes in the sampling matrix the voxels have zero value, that is there is a hole.
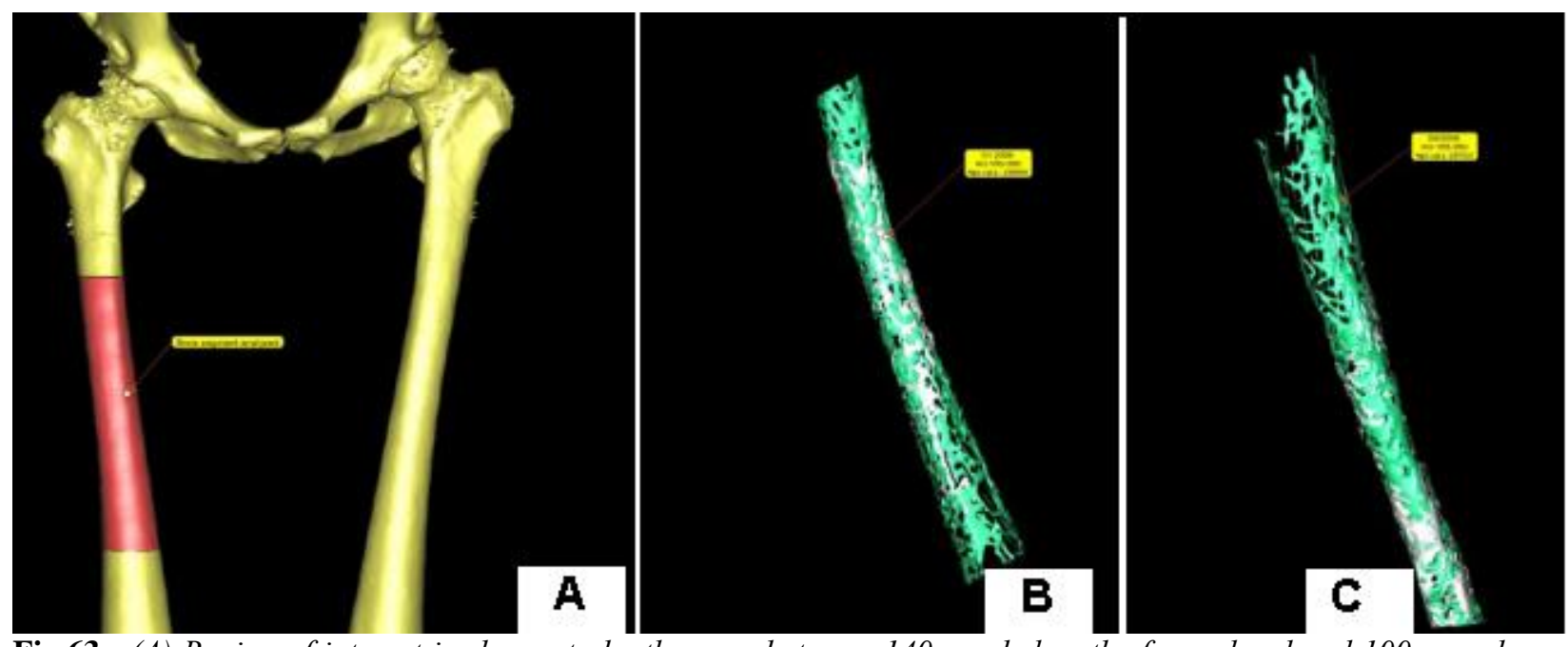

Fig 63 (A) Region of interest is chosen to be the area between $140 \mathrm{~mm}$ below the femur head and $100 \mathrm{~mm}$ above the knee joint. (B)Pt.3 in Jan. 2006. ROI for the interval 351 - 550 HU is shown as green and ROI for the interval $150-350 \mathrm{HU}$ can be seen as light grey through the first one. The volume of the former interval measures $11444 \mathrm{~mm}^{3}$. (C) Pt.3 in Sept. 2006. ROI for the same intervals as in panel B. The volume of the interval $351-$ $550 \mathrm{HU}$ measures $11331 \mathrm{~mm}^{3}$ 


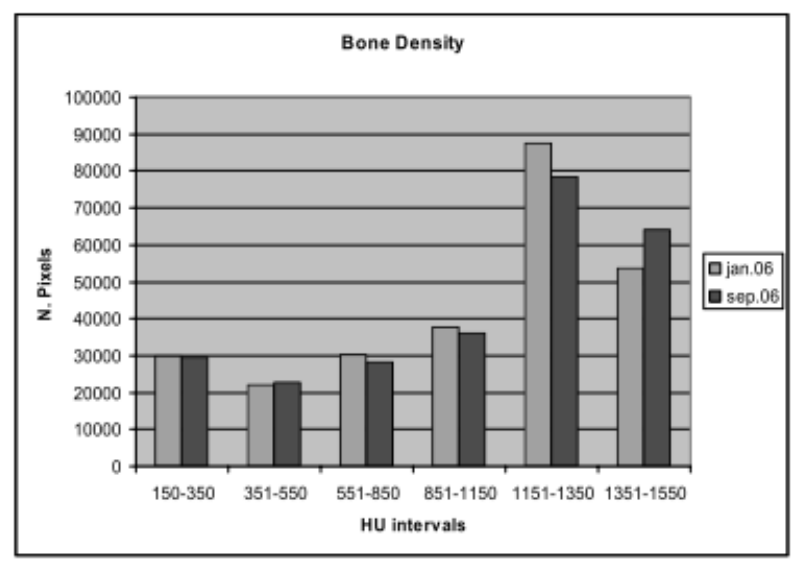

Fig 64 Number of pixels in each HU interval is compared for the dataset taken in Jan. 2006 and Sept. 2006 for Pt. 3

It is difficult to use the results to compare the shape of the tissue inside each HU interval. But the comparison of volume and density can be done as is shown in figure 6.3. There it can be seen that the volumes of the lower density intervals do not change much between January and September 2006 by Pt.3. But the upper two change. In the interval from 1151 to 1350 the number of voxels or the volume decreases and in the interval from 1351 to 1550 it increases just about the same amount. As the total volume remains constant this suggests that some of the voxels that where in the lower density interval in January are in September in the higher density interval indicating an increase in bone density in that time period. This suggests that the RISE therapy of DDM of the thigh has an influence on the bone mineral density.

\subsection{Modelling patella bone}

The patella bone is chosen as study volume to monitor changes in bone mineral density during FES treatment. The patella bone is a good sample to study changes in density; it is a small bone with triangular shape which articulates with the femur and covers and protects the knee joint from external applied force. Beside, since all four parts of the quadriceps muscle attach to the patella via the quadriceps tendon, the patella bone is a perfect candidate to study structural changes induced by quadriceps contraction. Indeed vastus intermedialis and rectus femoris muscle are attached to the base of patella while vastus lateralis and vastus medialis are attached to lateral and medial borders of patella respectively. The patella bone is attached to the tendon of the quadriceps femoris muscle, which in normal situation contracts to extend/straighten the knee.

In paraplegic patients with flaccid paralysis and therefore completely denervated the patella is unloaded; the only possible load is induced by the contractile activity generated in the muscle with FES treatment. Indeed the patella is outside the electrical field generated by the stimulation and therefore changes in density can be attributed exclusively to the contraction force transferred on the patella from the quadriceps muscle.

The bone mineral density changes in the patella during the stimulation trial are measured and analysed in following paragraphs.

\subsubsection{Patella segmentation and reslicing}

The information used to study the patella's bone are extract from the CT data, in fact the patella is isolated from the surrounding using the segmentation techniques described in the previous chapters. In general special attention is paid in order to gather data in a standardized form to allow comparison; therefore, before scanning, the patient's feet and ankles are bandaged together and both thighs line on the patient bed parallel to each other. In this way the muscles inside the field of view have similar orientation. This is important in the segmentation work and especially in the analysis of muscle shape (Paragraph 4.5). This arrangement is good enough to compare geometry and shape in large volumes such as the quadriceps muscles but not in small region of interest where even little displacement can influence remarkably the object orientation from time to time. This is the case for the patella which is small compared to the quadriceps muscle and it is positioned on top of the knee joint

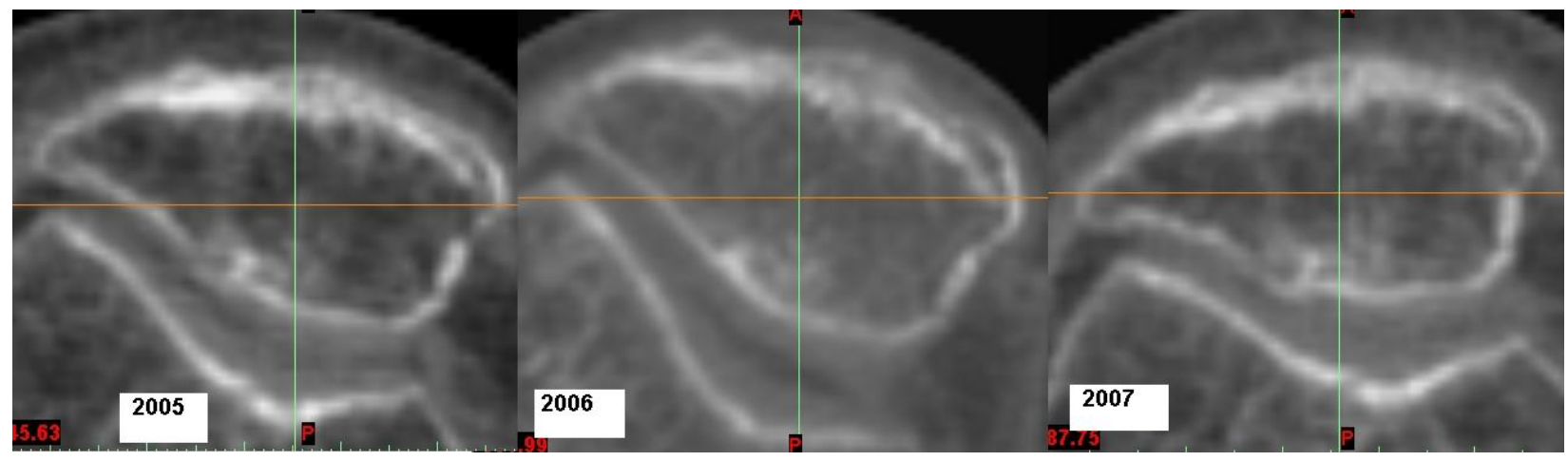

Fig 65 Three dataset from the same subject which show the different position of the patella from time to time 


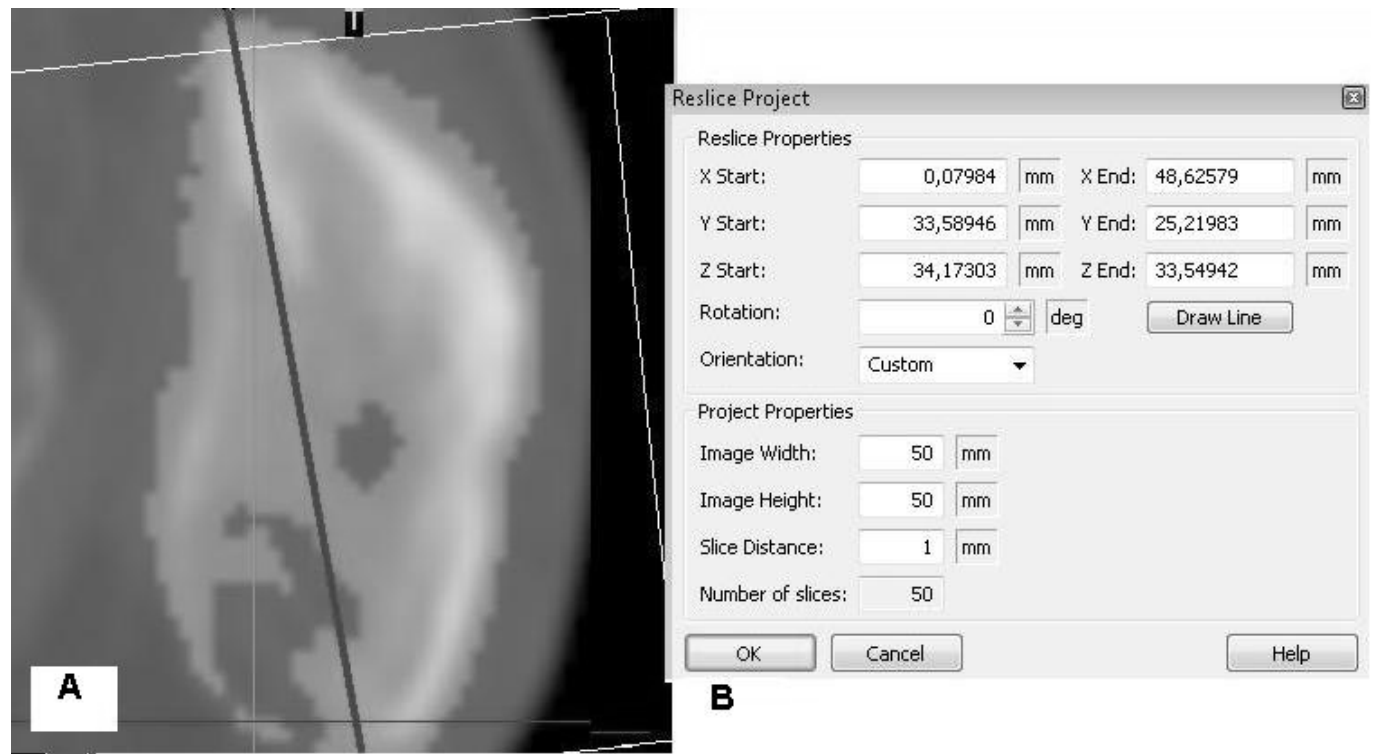

Fig 66 Reslicing process: (A) new scanning axes on the sagittal plane. (B) set up panel for the transformation matrix

when the thighs are lining horizontally and therefore sensible to small angular changes. Indeed the patella's scanned at different point of time result oriented differently in the coordinate system and makes difficult the cross sectional comparison.

The method developed to overcome this problem is based on project cropping and reslicing. A new data set is created cropping the original project which from the original 900-1000 slides is reduced to 50- 60 slides; the new data set is centered on the patella.

The patellas are oriented differently from time to time as showed in figure 65 . To adjust the position an image processing tool called reslice and developed in MIMICS [44] software is used. The reslicing process consist basically in re-scan the data along a customised direction with the result of producing a new set of images perpendicular to the new direction. This process is purely computational and therefore the patient isn't scanned again. The region of interest remains unchanged after reslicing but the images resolution may deteriorate a little bit.

The new scanning axes are drawn manually on the sagittal plane as shown in figure 66, A, and a transformation matrix is defined and applied to convert the original coordinate system in to the new one (Figure 66, B).

The images resolution in the data set is as good like in the original one but the number of slice reduce because

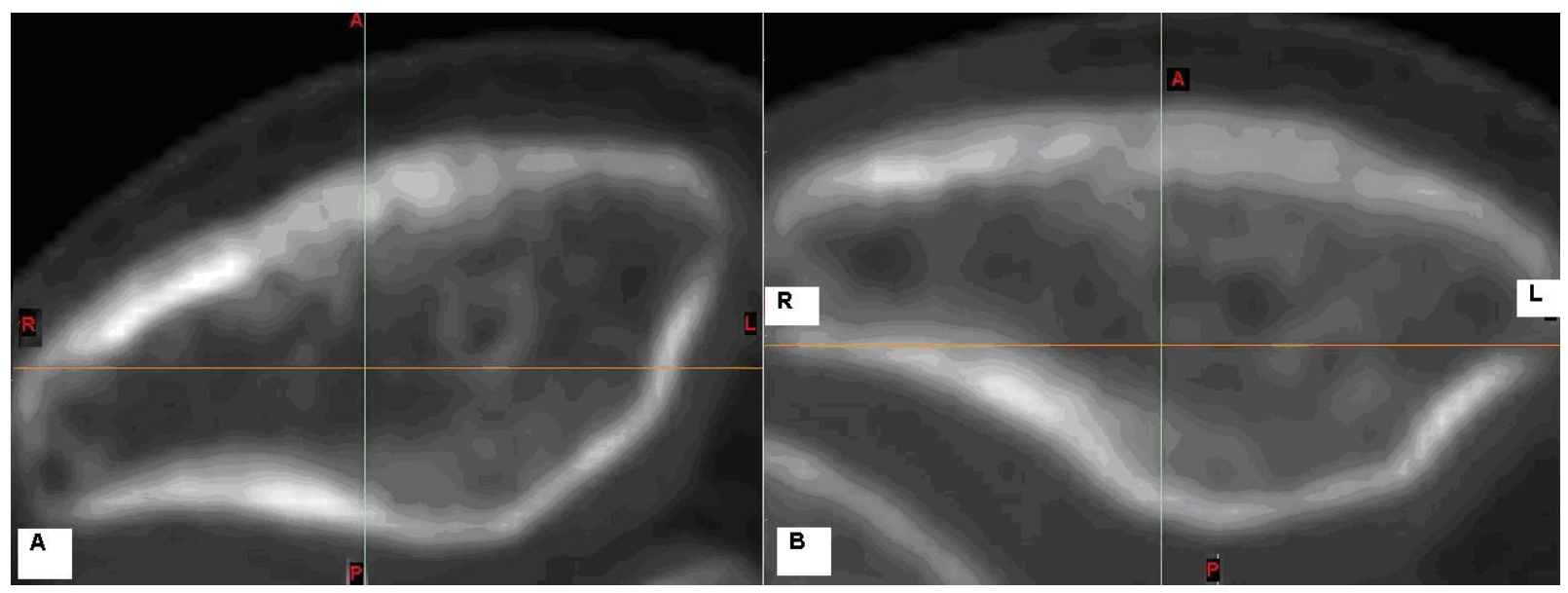

Fig 66 (A) Pre reslicing axial view of the patella. (B) Post reslicing axial view of the patella 


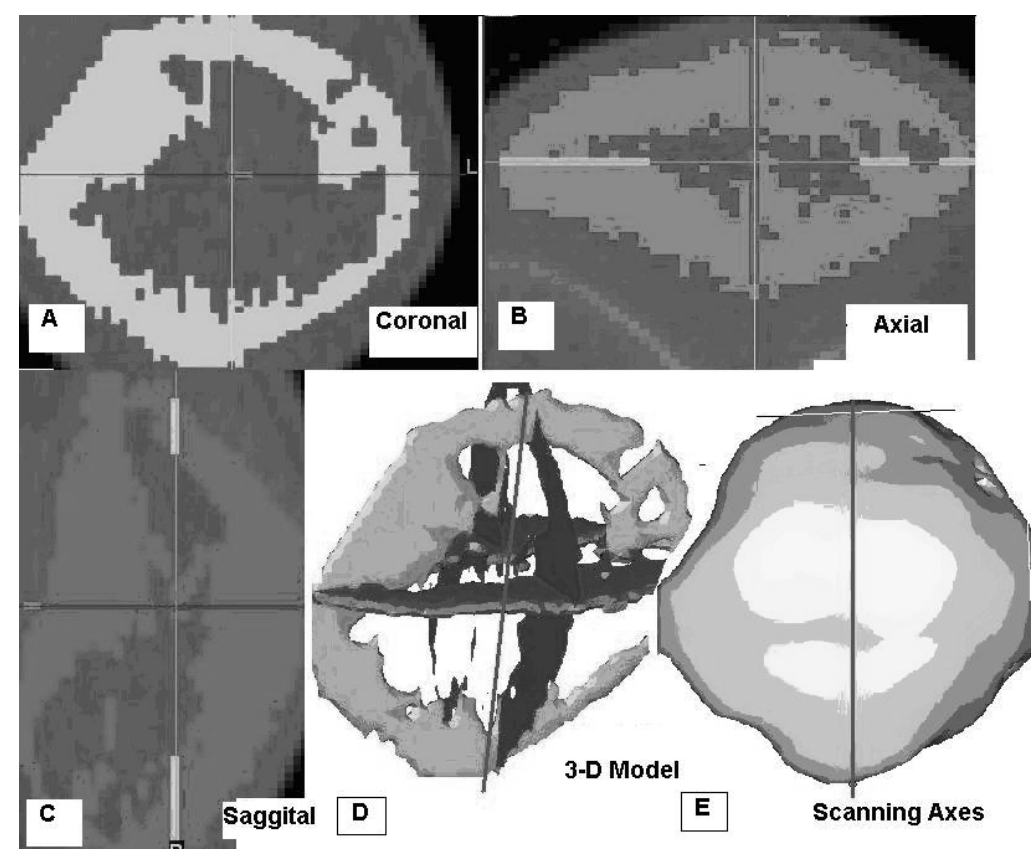

Fig 67 (A, B, C): $2 D$ segmentation masks: coronal, axial and sagittal. (D): $3 D$ rendering of segmentation mask. (E) $3 D$ model of the patella

the reslicing protocol have minimum slice increment of $1 \mathrm{~mm}$ while in the original protocol the slice increment was $0,625 \mathrm{~mm}$. In figure 67 pre and post resliced patella are seen, the resliced patella's have the large frontal side which is "parallel" to the r-1 axes (Figure 67, B).

The changes in bone density are analysed in the whole patella volume and in selected sections on the axial, sagittal and coronal planes. This approach can give information on bone density changes on the whole patella and also through the different directions.

Indeed the force distribution on the patella is not known in the specific case of denervated muscle treated with FES. The patella in paraplegic is unloaded expect for the induced muscle contraction, if there is a bone remodelling as effect of the stimulation it could be more developed in certain areas and along specific direction then in other. Therefore the changes in density are also measured on the three coordinate planes intersecting the patella centre; this can add information on how and where quadriceps contraction applies the force on the patella.

Using segmentation techniques four segmentation masks are created: whole patella, coronal plane, axial plane and sagittal plane. The 3D rendering of these masks with the scanning axes from the reslice process are showed in figure 67, D-E.

6.3.2 Conversion of $\mathrm{HU}$ values in bone mineral density

The next step to evaluate the bone density is convert the $\mathrm{HU}$ values contained in the segmentation masks in the respective bone mineral density values. Bone density is the amount of bone tissue in a certain volume of bone $(\mathrm{g} / \mathrm{cm} 3)$. Bone mineral density (BMD) is a test that measures the amount of calcium in a special region of bones. From this information, an estimate of the strength of the bones can be made. BMD helps predict the risk of a future fracture of the bone, measures the amount of bone mass, and also monitors the effectiveness of treatment. BMD can be measured using a special $\mathrm{x}$-ray technique called a quantitative computed tomography (QCT) or dual energy x-ray absorptiometry (DEXA). The normal, average bone mineral density is around $0.388 \mathrm{~g} / \mathrm{cm} 3$ in males and $0.290 \mathrm{~g} / \mathrm{cm} 3$ in females. Individuals with a BMD lower than $0.10 \mathrm{~g} / \mathrm{cm} 3$ need certain care [74]. The technique used in this work is called quantitative computed tomography (QCT).

Quantitative computed tomography (QCT) is an established method for the assessment of bone mineral density (BMD) in osteoporosis [75]. The technique requires calibration of $\mathrm{CT}$ numbers, and several differently designed calibration standards are being used for this purpose. Originally, the majority of the calibration standards contained solutions of K2HPO4 until have been observed problems in long-term stability of these devices, mainly due to the formation of air bubbles and the corresponding errors in the calibration process [76]. To overcome these problems, calibration standards based on solid calcium hydroxyapatite are used.

The calibration standards used to convert $\mathrm{Ct}$ number in bone mineral density was the QCT-3000 phantom. A QCT scan of an anatomical site is acquired in conjunction with a calibration phantom that is 


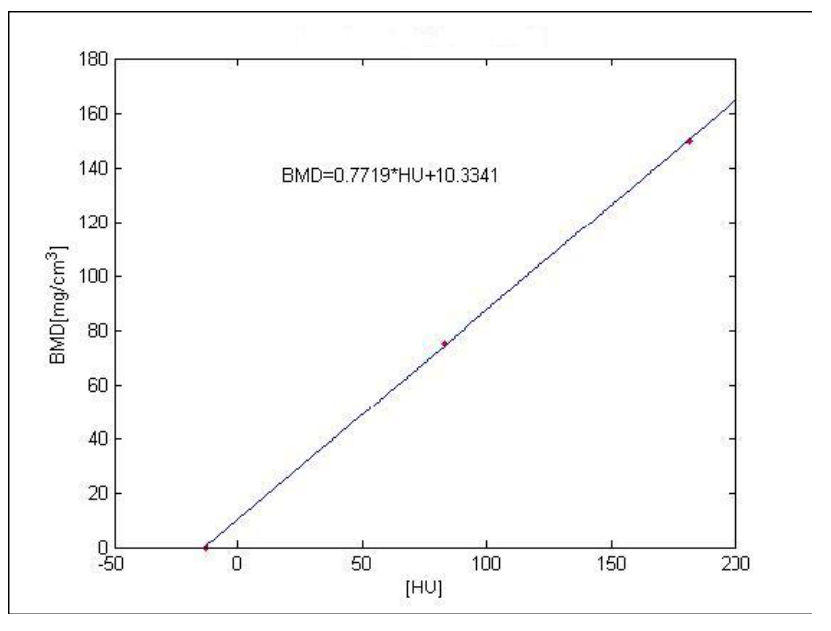

Fig 68 The relationship between $B M D$ and $H U$ : estimated from the three materials in the QCT3000 phantom [73]

composed of a small number (usually three to seven) of materials, each material having a specific "bone equivalent" value. A quantitative interpolation between the values of the portion of the image associated with the region of interest with the portions associated with the phantom materials allows a bone-equivalent value in $\mathrm{g} / \mathrm{cm} 3$ to be derived. The relationship between concentration of calcium hydroxyapatite and $\mathrm{HU}$ is linear [77]. In figure 6.8 a graph of the concentration of calcium hydroxyapatite of the materials in the phantom versus $\mathrm{HU}$ can be seen, together with the equation for the relationship between BMD and HU. The linear relationship between $\mathrm{BMD}$ and $\mathrm{Ct}$ number is:

$$
B M D=0,7719^{*} H U+10,3341
$$

\subsection{Bone mineral density measurement on the patella}

An algorithm was designed in Matlab that scaled the data and converted it from HU to BMD and display the bone mineral density distributions in the three segmentation planes [Appendix 2]. In figure 69 these distribution are calculated for a healthy subject. The BMD values are colour coded from blue to red depending from the density value: pixels with low BMD in blue, pixels with high BMD in red.

Can be noticed in the control patella a less dense area on the cortical bone facing the knee joint (patella back side) compared with the front side; this can be seen clearly in the axial and coronal plane. This probably is explained from the fact that the patella function is protecting the knee joint from external force and therefore the front side which is in general more exposed to these solicitations and to unexpected stress (e.g. fall or accident) develops stronger bone structure. Sectional BMD distribution on coronal, axial and sagittal plane are made in the patella from the three paraplegic patient's ongoing FES treatment. The bone mineral density changes can be evaluated and compared among five years monitoring. BMD distribution in the coronal, axial and sagittal plane are respectively shown in figure 70-72.

\section{Coronal view}

In patient 1 the density seems to reduce slightly from 2003 to 2006 (lesser red coded pixels) but in 2007 and 2008 the distribution appears a bit denser.

In patient 2 the density reduces sensibly until 2005; after that the situation remains basically unchanged.

Changes are also measured in patient 3 where on the coronal plane the density is generally not increasing but have negative peaks in 2005 and 2008 with cortical bone disappearing in large part from the surface.

\section{Axial view}

In patient 1 a reduction in mineral density is noticeable in 2005 and as well a sensible density increase in 2007. In patient 2 the situation remains unchanged in the outer part but decrease in the inner section.

Also in patient 3 the situation is rather stable during the monitored period without any positive or negative remarkable density changes.

\section{Sagittal view}

In patient 1 a loss in bone density is noticeable in 2005 and 2006 but like seen in the axial view the bone density increase in 2007. In patient 2 and 3 not major changes can be appreciated from the sagittal view.
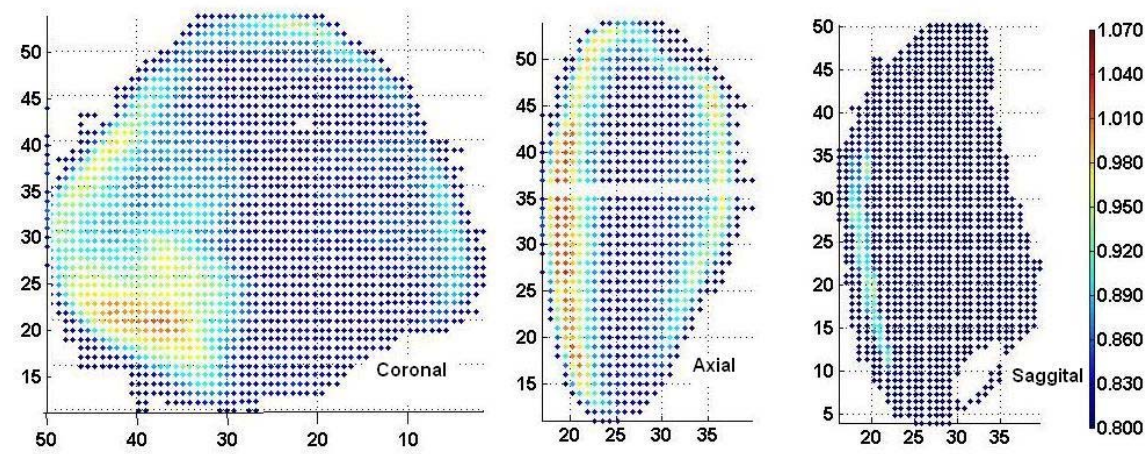

Fig 69 Patella coronal, axial and sagittal BMD distribution of a control subject 


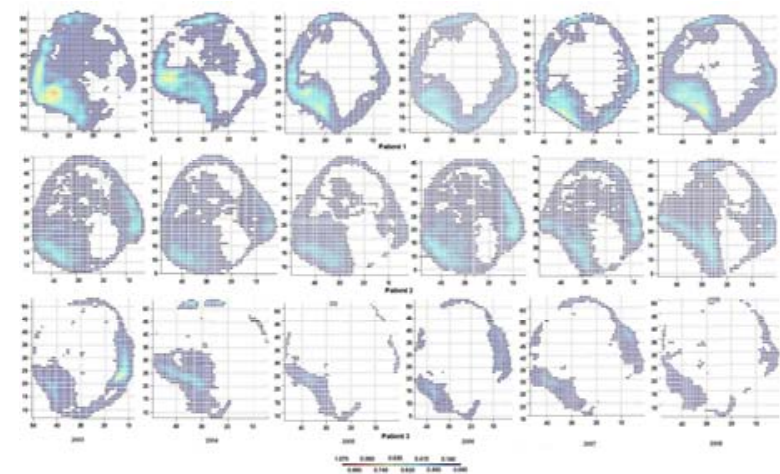

Fig 70 Patella coronal view: first row patient 1 (10 months denervated in 2003), second row patient 2 (4 years denervated in 2003) and third row patient 3 (7 years denervated in 2003

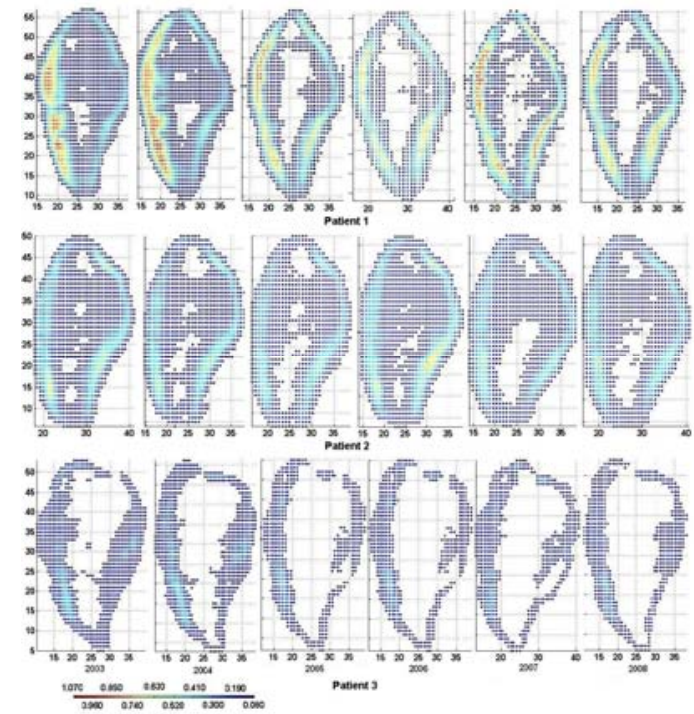

Fig 71 Patella axial view: first row patient 1, second row patient 2 and third row patient 3

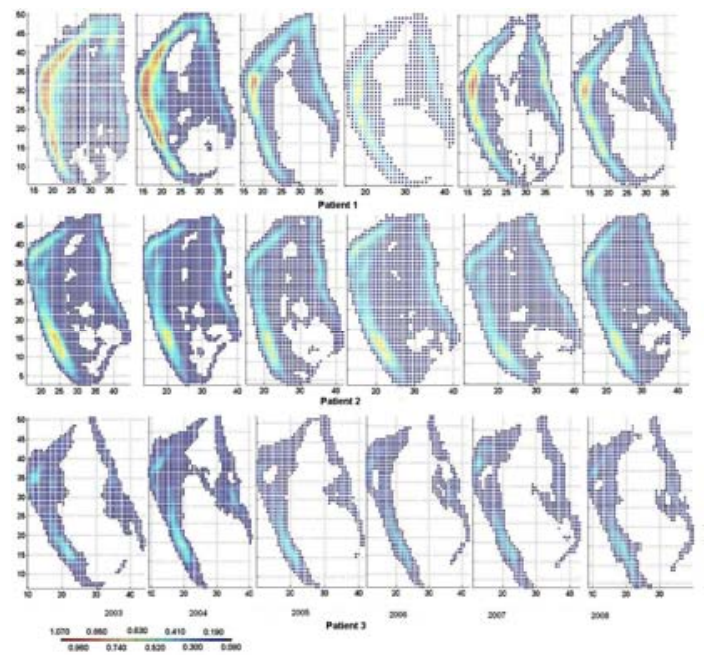

Fig 72 Patella sagittal view: first row patient 1, second row patient 2 and third row patient 3

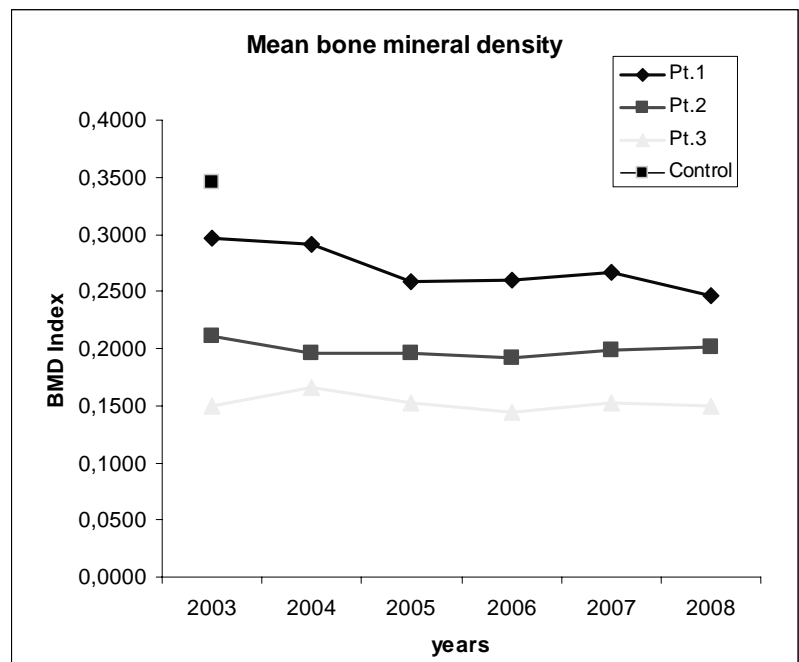

Fig 73 Mean bone mineral density trend

To quantify the overall bone mineral content in the patella, the mean bone mineral density value is calculated using the following formula:

MBMD $=\left(\frac{\sum B M D}{\text { length }}\right)$

Where

$\sum B M D=$ Sum of all BMD values in the data set

Length: number of pixels in the data set

The mean bone mineral density trends for the three patients are displayed in figure 73 .

The first impression from the results displayed in figure 73 is that mineral density isn't changing remarkably during the FES treatment. The three patellas remain more or less on their initial MBMD levels, though these levels are quite different because of the diverse initial conditions in 2003 (10 months denervation patient 1,4 years degeneration patient 2 and 7 year degeneration patient 3 ). In patient 2 which actually started the FES treatment in 2004 can be notice the trend changing before and after FES; in fact MBMD continue to decrease from 2003 to 2004 while after 2004 mineral loos is stopped and even increases a little bit.

The fact that FES treatment stop bone mineral loss can be demonstrated still more clearly analysing figure 74 and 75 .

In figure 74 the axial view of patella's from different subjects are compared: the bone mineral distribution in the 6 years denervated subject treated with FES is much better compared with both 4 and 7 years 


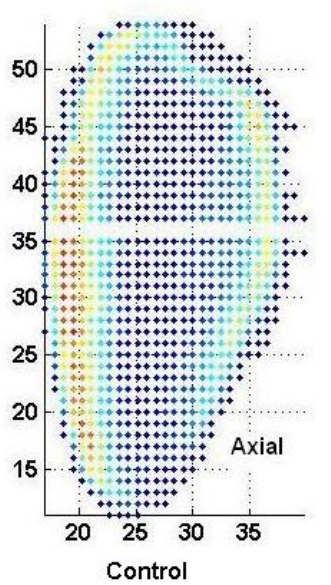

Mean BMD value: 0.281 gricm3
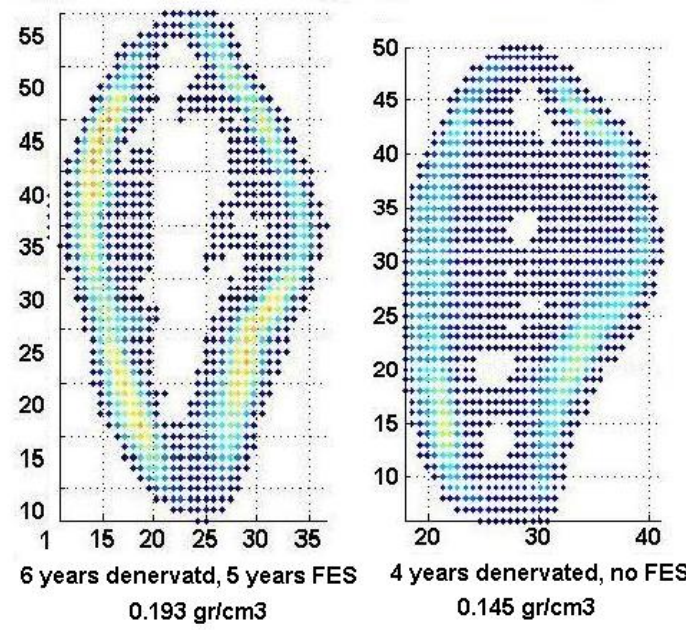

0.145 gricm 3

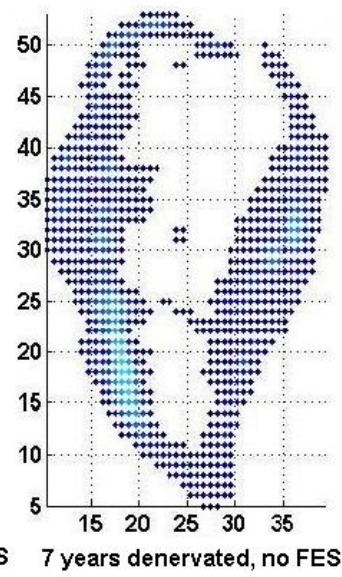

0.106 gricm 3

$$
\begin{array}{ccccccc}
1.070 & 0.850 & 0.630 & 0.410 & 0.190 \\
\hline 0.960 & 0.740 & 0.520 & 0.300 & 0.080
\end{array}
$$

Fig 74 Axial view and respective BMD mean value from four subjects with different initial clinical conditions and FES treatment

denervated subjects but not treated with FES. Indeed the BMD mean values are respectively $0.193,0.145$ and $0.106 \mathrm{gr} / \mathrm{cm} 3$.

In figure 75 the patellas from different subjects are 3-D modelled and visualized. The different BMD levels are mapped on the patella volume. From the same subject is shown in panel $\mathrm{B}$ and $\mathrm{C}$ respectively the situation after 10 months from denervation and then after 5 years of denervation with 4 years of FES. In panels D, $\mathrm{E}$ and $\mathrm{F}$ from the same subject are shown respectively

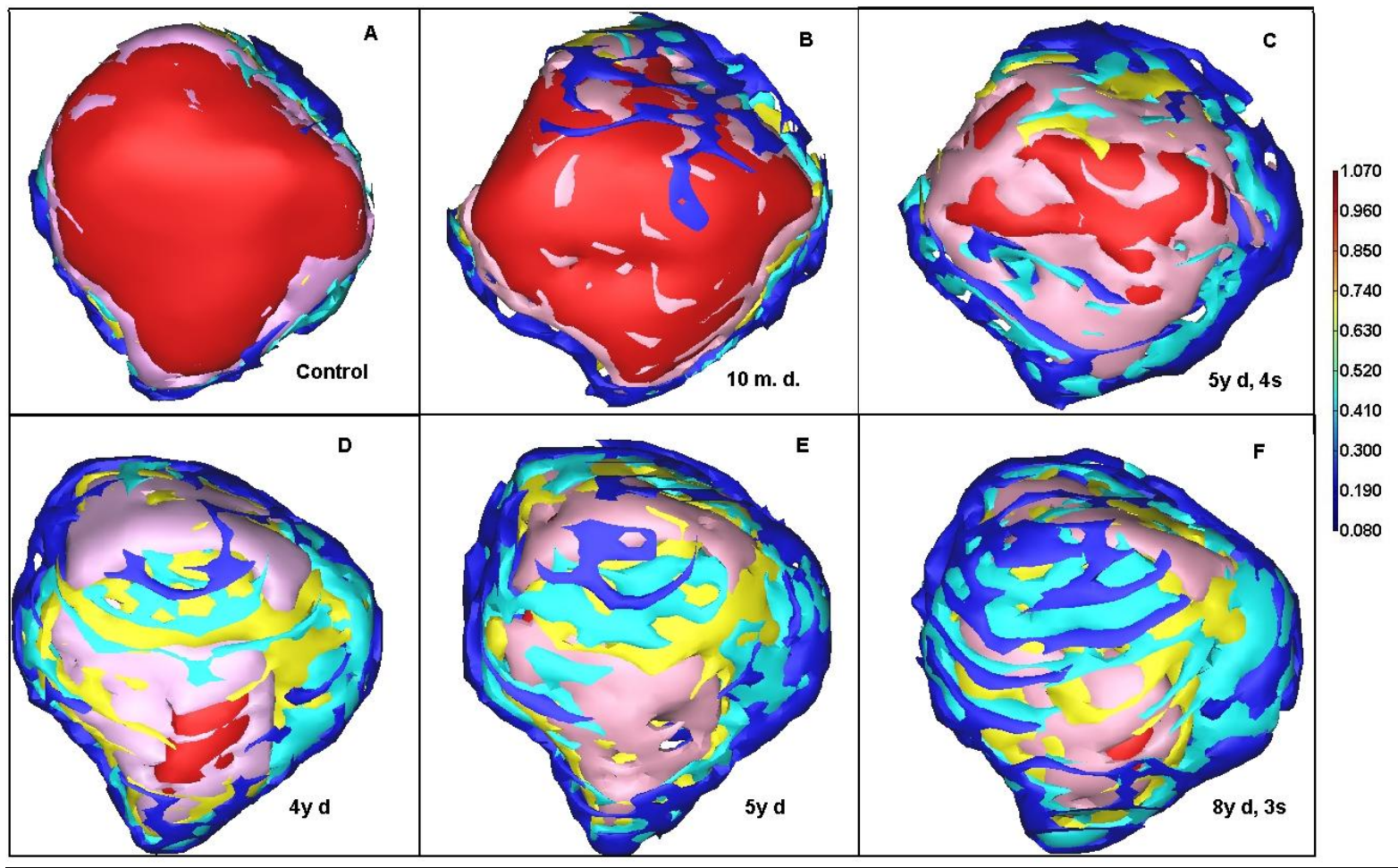

Fig 75 Bone Mineral Density mapping on the patella 3D volume for subject's with different initial clinical conditions and FES treatment: (A) control, (B) 10 months denervated, (C) 5 years denervated and 4 years FES, (D) 4 years denervated, (E) 5 years denervated, $(F) 8$ years denervated and 3 years FES 


\section{D Modelling and monitoring of denervated muscle under FES}

European Journal Translational Myology - Basic Applied Myology 2011; 21: 31-94

the patellas after 4 years denervation, 5 years denervation and 8 years denervation with 3 years of FES treatment.

Comparing $\mathrm{B}$ and $\mathrm{C}$ it is clear the developed bone loss in the patella even though the patient was treated with FES in mean time. On the other hand are also obvious the differences in BMD between the two patellas in $\mathrm{C}$ and $\mathrm{E}$ which have the same denervation time but in $\mathrm{C}$ the quadriceps muscle were treated with FES while in E not. Finally comparing $\mathrm{E}$ and $\mathrm{F}$ can also be appreciate a slightly improvement in BMD in the patella which muscle were stimulated for three years.

\section{DISCUSSION}

The reversibility from the degeneration status in long term denervated muscle has been largely demonstrated during many years of clinical trials and validated through many striking basic research work.

The present study uses a novel approach to further validate the clinical rehabilitation method and research correlations between different effects occurring in muscle and bone during the FES treatment. The study demonstrates qualitatively and quantitatively the structural changes occurring in muscle and bone due FES treatment. The main results and relationship are summarised graphically in figure 76 .

The methodology used in this work it is based on gathering medical images from helical CT-scans and employ modelling tools to process and develop data. On the reliability of the measurement provided in this work some comments are required. The modelling isn't a precise discipline and therefore several constraint and approximation are necessary and have been adopted in this work. Special attention was paid to the proper use of HU numbers for tissue discrimination and the employment of segmentation processes to separate different muscles.

The HU values are susceptible of variation from one data set to another and along the same scanning at different cross sections. Though calibration tools have been used to quantify $\mathrm{HU}$ oscillation and large $\mathrm{HU}$ interval to account them, the direct use of HU number remain unreliable parameter in the analysis of small variation. Patient position and scanning protocol should be further standardised to reduce and quantify HU number variability.

Segmentation processes are largely used in the study to represent muscle, bones and other tissues. The segmentation is mostly an automatic process, based on a defined computational strategy and aiming to discriminate elements in a complex image. The automatic approach work well in segmenting different tissues like muscle-fat, muscle-bone or corticaltrabecular bone, but it is not effective in the separation

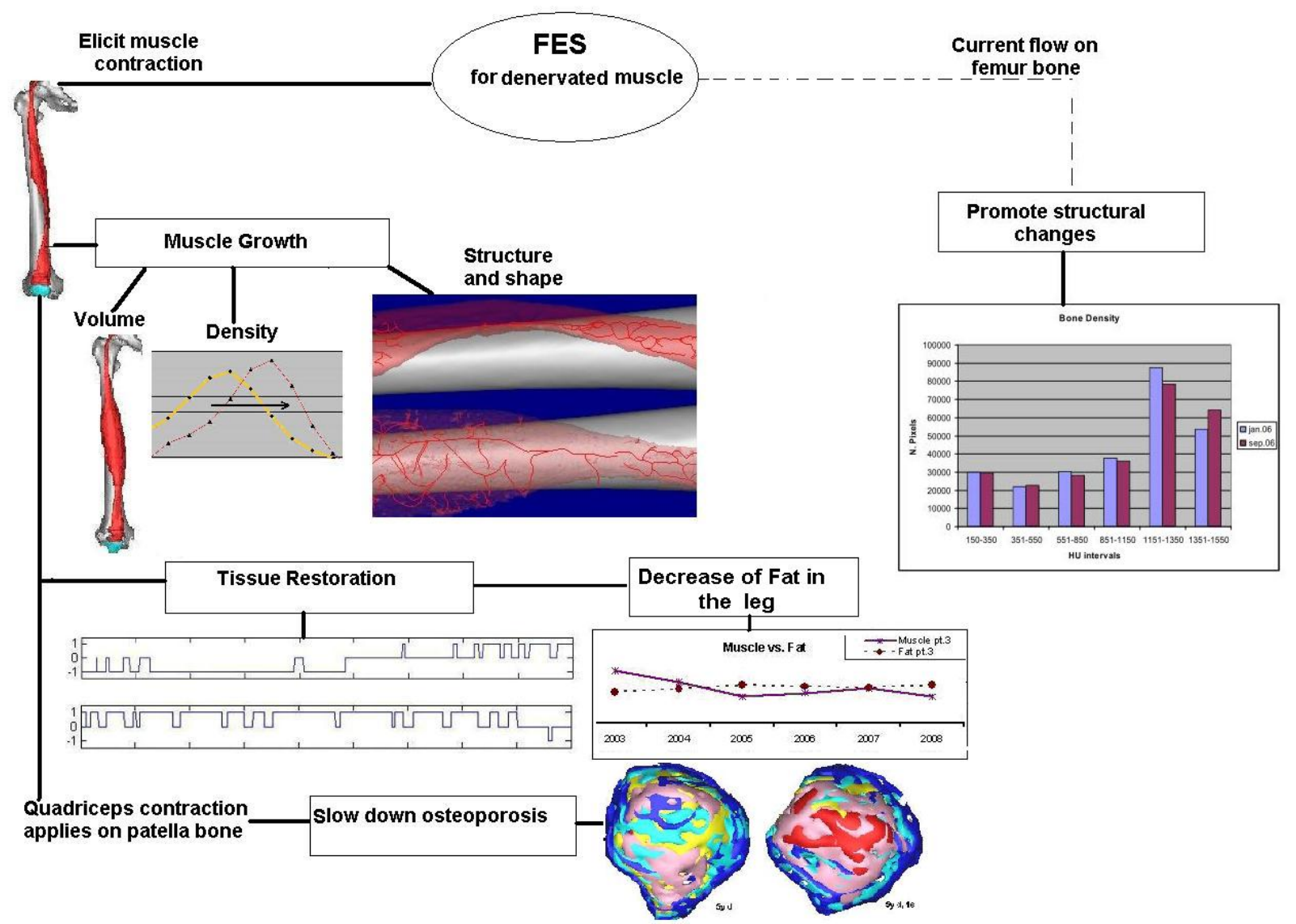

Fig 76 Graphical summary of the main results and relationships 


\section{D Modelling and monitoring of denervated muscle under FES}

European Journal Translational Myology - Basic Applied Myology 2011; 21: 31-94

of muscle bellies. Indeed the muscle-muscle segmentation it is usually performed manually using special editing tools; of course a good contrast on the medical image is required in order to distinguish one belly from another but in the degenerate muscle this maybe not be enough for a proper discrimination. This is the case for the segmentation of the quadriceps where the muscle bellies endings (proximal and distal parts) are not distinguishable on the cross section. The compromise used was to reduce the region of interest to a volume where the bellies are well defined (5. MODELLING AND ANALYSIS OF QUADRICEPS $M U S C L E)$. In this way the segmentation is rather precise and allows qualitative indications on the growth within the different quadriceps muscle. In the future an improved segmentation tools should be developed to enhance the tissue discrimination within muscles.

\subsection{Use of the results and future work}

The current flow elicits contraction in denervated muscle fibres allowing growth. The parameters that can directly quantify the growth in muscles are volume and density. This study demonstrates that also geometrical changes can be quantified and correlated to the restoration process. In fact the change in rectus femoris shape during grow suggest a relationship between restoration of contractile activity and local decrease of curvature index (4. MODELLING AND ANALYSIS OF RECTUS FEMORIS MUSCLE ). These findings could be used to contribute in the modelling of the denervated muscle behaviour: for example simulating different electrode geometry and activation pattern with the changes in muscle shape in order to find optimal electrode geometry and electrical parameters which optimise the muscle growth.

The restoration process which is measured locally analysing the tissue composition within the muscle belly demonstrate that the quadriceps muscle don't respond in the same way to the treatment. While remarkable grow and restoration is measured in some belly, changes in the others are minimal or not at all present. The reason for this difference in grow among quadriceps muscle must be researched in the patient leg morphology and in the electrode position. Indeed the adipose tissue surrounding the muscles can plays an important role in the treatment effectiveness avoiding the electrical stimulus to reach properly the muscle fiber. Beside the muscle position inside the leg can be different from a patient to another. A way to improve and distribute better the electrical stimulus along the muscles can be obtained by knowing exactly where the target muscle is located below the patient skin. This idea could be implemented using several $\mathrm{Ct}$ or MRI cross sections along the region of interest and projecting the stimulation area on the skin as it is showed in figure 77. Of course the surface on the skin which covers properly the target muscles will be different in every patient as displayed in the figure. Therefore the surface electrodes should be available in different size and positioned on the patient thigh according to the muscle-skin map.

The direct and indirect effects on the bone structure due FES treatment have been investigated.

In general, current flow influences the bone density in femur; these changes are small and the data from the three patients not statistically relevant. Anyway the data is important for future work since the bone loss treatment in SCI patient is an unsolved issue. For this reason to validate the positive FES action on the bone remodelling, the region of interest where the bone density is investigated should be designed ad hoc. Indeed the femur is too big to be monitored as one volume; changes of opposite signs from different locations can annul important local variation. In the future the bone should be monitored locally taking into account the current flow pattern produced by the surface electrode on the specific muscle geometry.

Structural changes in patella bone are measured as indirect effect of the electrical stimulation. Also in this case the changing and data aren't statistically relevant but the indications provided in this study very important. Indeed the patella in SCI patient is completely unloaded and loses density irretrievably with the time like other bones. The current flow doesn't play a role in the density variation since the

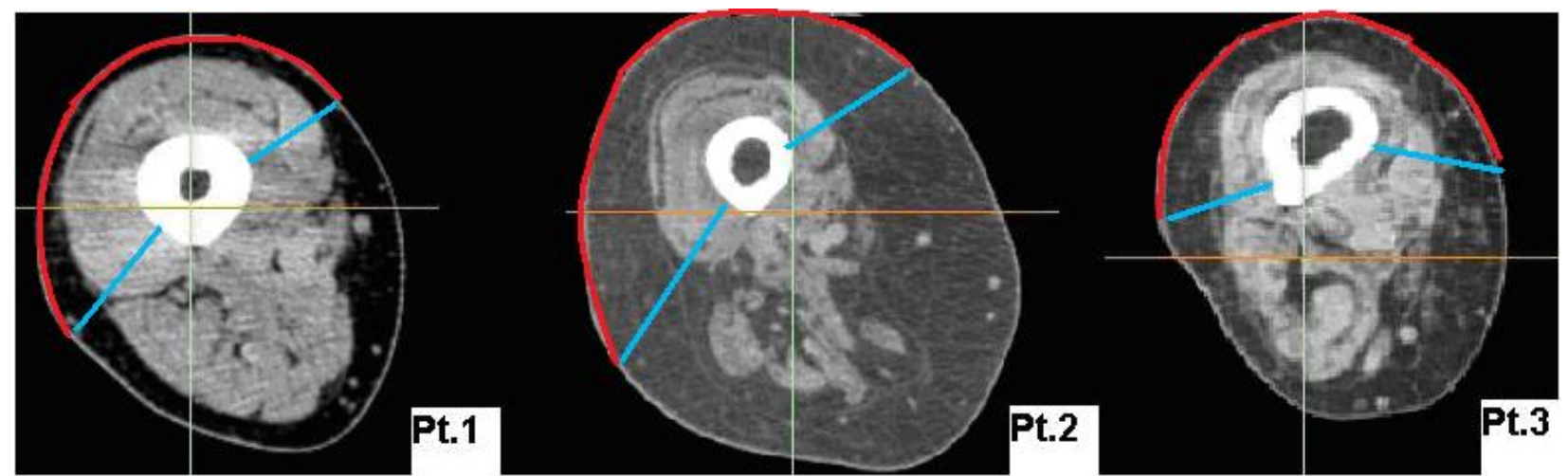

Fig 77 Projection of the quadriceps muscle on the skin (in red) showing the different surface electrode required to cover the properly the stimulation target 


\section{D Modelling and monitoring of denervated muscle under FES}

European Journal Translational Myology - Basic Applied Myology 2011; 21: 31-94

patella is far away from the stimulated area. The only possible factor that can explain the slowing down of bone loos is the action-force applied from the quadriceps muscle when they contract. This special model should be used to quantify and correlate forcedirection and bone remodelling

\section{Abbreviations}

$\mathrm{AP}$, action potential; $\mathrm{BMC}$, bone mineral content; BMD, bone mineral density; CT, computed tomography; DC, direct current; DDM, denervated degenerated muscle; DICOM, digital imaging and communications in medicine; ES, electrical stimulation; FES, functional electrical stimulation; HU, Hounsfield unit; LMN: lower motor neurone; MR,Magnetic resonance imaging ; PDA, personal digital assistant; PC, personal computer ; Pixel, picture element; QCT,quantitative computed tomography; ROI,region of interest SCI, spinal cord injury; Voxel,volume element; RM, root mean square error ; RP, Rapid Prototyping; UV, Ultraviolet; $\mu$, X-ray linear attenuation coefficient; I0, incident beam intensity; It, transmitted beam intensity; 3D, three dimensions

\section{Acknowledgements}

This work was possible because of the support and the cooperation of many people.

I would like to thank and express my appreciation to my reviewers: Prof. Rattay, Prof. Mayr and Dr. Helgason

The RISE clinical trial in Iceland has been possible thanks to the work of Sigrún Knútsdóttir, Vilborg Guðmundsdóttir, Páll Ingvarsson and Stefán Yngvason from the department of Rehabilitation Medicine Landspitali-University Hospital.

The PhD idea developed at Department for Research and Development, Division of Clinical Engineering in Landspitali; thanks to Brynjar Vatnsdal and Linda Birgisdóttir for their help in the computational work. Also thanks to Björg Guðjónsdóttir and Guðfinna Halldórsdóttir, with Linda all students of University of Reykjavik, for fruitful discussions and exchange of experience.

I appreciate the support I received from Landspitali during the study, for this I thank Porgeir Pálsson and Pórður Helgason.

Thanks to the employees at Centre of Biomedicine Technique and Physic, Medical University of Wien AKH for offering always welcome, help and friendship during my multiple visits. Particularly I wish to thanks Winfried Mayr, Thomas Mandl and Martin Reichel. This work was supported by RANNÍS the Icelandic Centre for Research, by the Science Fund of Landspítali, by the Students Innovation Fund (NSN) and by Health Technology Venue (HTV) which I greatly appreciate.

\section{Corresponding Author}

Paolo Gargiulo, Dept. Science, Education, Innovation, Landspitali University Hospital and Dept. Biomedical Engineering, University of Reykjavik, Iceland. E-mail: paologar@landspitali.is

\section{References}

[1] Moe JH, Post HW. Functional electrical stimulation for ambulation in hemiplegia. J Lancet 1962; 82: 285-288

[2] McNeal DR. 2000 Years of electrical stimulation. In Hambrecht and Reswick (eds): Functional Electrical Stimulation. Marcel Dekker Inc., New York, 1977;3-35.

[3] Gold GR. Cardiac pacing-from then to now. Medical Intsrumentation 1984; 18: 15-21.

[4] Greatbatch W. Origins of the implantable cardiac pacemaker. J Cardiovasc.Nurs. 1991; 5: 80-85.

[5] Møller M, Arnsbo P, Asklund M, Christensen PD, Gadsbøll N, Svendsen JH, Klarholt E, Kleist KE, Mortensen PT, Pietersen A, Simonsen EH, Thomsen PE, Vesterlund T, Wiggers R. Quality assessment of pacemaker implantations in Denmark. Europace 2002; 4(2):107-12.

[6] Cochlear implants in adults and children. NIH Consens.Statement 1995; 13: 1-30

[7] Liberson WT, Holmquest HJ, Scot D, DOW M. Functional electrotherapy: stimulation of the peroneal nerve synchronized with the swing phase of the gait of hemiplegic patients. Arch.Phys.Med.Rehabil. 1961; 42: 101-105.

[8] Popovic MR, Keller T, Pappas IP, Dietz V, Morari M. Surface-stimulation technology for grasping and walking neuroprosthesis. IEEE Eng Med Biol.Mag. 2001; 20: 82-93.115.

[9] Scott TR, Peckham PH, Keith MW. Upper extremity neuroprostheses using functional electrical stimulation. Baillieres Clin.Neurol. 1995; 4: 57-75.

[10] Glenn WW, Phelps ML, Elefteriades JA, Dentz B, Hogan JF. Twenty years of experience in phrenic nerve stimulation to pace the diaphragm. Pacing Clin.Electrophysiol. 1986; 9: 780-784.

[11] Carraro U, Catani C, Saggin L, Zrunek M, Scabolcs M, Gruber H, Streinzer W, Mayr $\mathrm{W}$, Thoma H: Isomyosin changes after functional electrostimulation of denervated sheep muscle. Muscle Nerve 11:1016-1028, 1988.

[12] Valencic V, Vodovnik L, Stefancic M, Jelnikar T: Improved motor response due to chronic electrical stimulation of denervated 


\section{D Modelling and monitoring of denervated muscle under FES}

European Journal Translational Myology - Basic Applied Myology 2011; 21: 31-94

tibialis anterior muscle in humans. Muscle Nerve 1986; 9: 612-617

[13] Lomo T, Westgaard RH, Hennig R, Gundersen $\mathrm{K}$ : The response of denervated muscle to longterm electrical stimulation. in Carraro U, Angelini C, (eds): Cell Biol-ogy and Clinical Management in Functional Electro Stimulation of Neurones and Muscles. Padova, Italy, CLEUP Editore, 1985, pp 81-90.

[14] Zrunek M, Bigenzahn W, Mayr W, Unger E, Feld-ner-Busztin H: A laryngeal pacemaker for inspiration controlled direct electrical stimulation of denervated posterior cricoarytaenoid muscle in sheep. Eur Arch Otorhinolaryngol 1991; 248 (8): 445-448.

[15] Kern et al. 1999 Kern H, Hofer C, Strohhofer M, Mayr W, Richter W, Stöhr H: Standing up with denervated muscles in humans using functional electrical stimulation. Artif Organs 1999; 23 (5): 447-452.

[16] Carraro U, Rossini K, Zanin ME, Rizzi C, Mayr $\mathrm{W}$, Kern H: Induced Myogenesis in LongTerm Permanent Denervation: Perspective Role in Functional Electrical Stimulation of Denervated Legs in Humans. Basic Appl Myol 2002; 12 (2): 53-63

[17] Ashley Z, Sutherland H, Lanmu“ller H, Russold MF, Unger E, Bijak M, Mayr W, Boncompagni S, Protasi F, Salmons S, Jarvis JC. Atrophy, but not necrosis, in rabbit skeletal muscle denervated for periods up to one year. Am J Physiol Cell Physiol 292: C440-C451, 2007; doi:10.1152/ajpcell.00085. 2006

[18] H Kern,C Hofer, M Modlin, W Mayr, V Vindigni, S Zampieri, $\mathrm{S}$ Boncompagni, $\mathrm{F}$ Protasi and U. Carraro Stable muscle atrophy in long-term paraplegics with complete upper motor neuron lesion from 3- to 20-year SCI, Spinal Cord (2008) 46, 293-304

[19] Simona Boncompagni, Helmut Kern, Katia Rossini, Christian Hofer, Winfried Mayr, Ugo Carraro. Structural differentiation of skeletal muscle fibers in the absence of innervation in humans, PNAS 2006

[20] Kern H, Hofer C, Modlin M, Forstner C, Raschka-Ho"gler D, Mayr W et al. Denervated muscles in humans: limitations and problems of currently used functional electrical stimulation training protocols. Artificial Organs 2002; 26: 216-218.

[21] Kern H, Salmons S, Mayr W, Rossini K, Carraro U (2005) Recovery of long-term denervated human muscles induced by electrical stimulation. Muscle Nerve 31:98-101
[22] Kern H, Boncompagni S, Rossini K, Mayr W, Fano ' G, Zanin ME et al.. Long-term denervation in humans causes degeneration of both contractile and excitation-contraction coupling apparatus that can be reversed by functional electrical stimulation (FES). A role for myofiber regeneration? J Neuropath Exp Neurol 2004; 63:919-931

[30] Modlin M, Forstner C, Hofer C, Mayr W, Richter W, Carraro U, Protasi F, Kern H Functional electrical stimulation of denervated muscles: first results of a clinical study. Artificial Organs 2005; 29: 203-206

[24] Hofer C, Mayr W, Stoehr H, Unger E, Kern $\mathrm{H}$ : A stimulator for functional activation of denervated muscles. Artif Organs 2002; 26 (3): 276-279.

[50] Winfried Mayr, Christian Hofer, Manfred Bijak, Dietmar Rafolt, Ewald Unger, Martin Reichel, Stefan Sauermann, Hermann Lanmueller and Helmut Kern(1), Functional Electrical Stimulation (FES) of Denervated Muscles: Existing and Prospective Technological Solutions Basic Appl Myol 12 (6): 287-290, 2002

[26] Hodgkin A.L. and Huxley A.F.. A quantitative discription of membrane current and ist application to conduction and excitation in nerve. J. Physiol. Lond. 117:500-544. 1952.

[27] Rattay F. Electrical nerve stimulation: theory, experiments and applications. Wien: SpringerVerlag, 1990.

[28] Reichel Martin, Funktinelle Elektrostimulation denervierter Skelettmuskulatur - Modellbildung und Simulation (Dissertation), Institut für Analysis und Technische Mathematik, TUWien 1999.

[29] Martinek Johannes, Analyse der Aktivierung von denervierten Muskelfasern im menschlichen Oberschenkel bei Funktioneller Elektrostimulation (Diplomarbeit), Institut für Analysis und Technische Mathematik, TUWien 2003.

[30] Thomas Mandl, Medical Image Segmentation for calculating effects of muscular anisotropy during Functional Electrical Stimulation (Diplomarbeit), Institut für Analysis und Technische Mathematik, TU-Wien 2005.

[31] M. Johannes, Stickler Y, Martin Reichel, Rattay F. A new approach to stimulate HodgkinHuxley like with Comsol Multiphysics (FEMLAB),. 9th Vienna international workshop on FES(2007) pag. 163-16

[32] Reichel M, Johannes M, Mandl T, Mayr W, Rattay F. Development of modelling FES in 


\section{D Modelling and monitoring of denervated muscle under FES}

European Journal Translational Myology - Basic Applied Myology 2011; 21: 31-94

denervated muscle. 9th Vienna international workshop on FES (2007) pag. 159-162

[33] Thomas Mandl, Martin Meyerspeer, Martin Reichel, Helmut Kern, Christian Hofer, Winfried Mayr, and Ewald Moser. Functional Electrical Stimulation of Long-term Denervated, Degenerated Human Skeletal Muscle: Estimating Activation Using T2Parameter Magnetic Resonance Imaging Methods Artificial Organs 2008; 32(8): 604608

[34] McCollough, CH, Morin, RL. The technical design and performance of ultrafast computed tomography. Radiol Clin North Am 1994; 32:521.

[35] www.gehealthcare.com

[36] The Unreliability of CT Numbers as Absolute Values. Clifford Levi, Joel E. Gray, Edwin C. McCullough, Robert, R. Hattery AJR 139:443447, September 1982

[37] Quasar multi-purpose body phantom user's guide. Modus medical devices Inc.page 15, 2003.

[38] Qct-3000 bone densitometry user's guide. Image Analysis, pages 2-6.

[39] McCullough EC, Morin RL. CT-number variability in thoracic geometry. AJR Am J Roentgenol 1983;141:135-140,

[40] Zhao B, Yankelevitz D, Reeves A, Henschke C. Two-dimensional multi-criterion segmentation of pulmonary nodules on helical CT images. Med Phys 1999; 26:889-895

[41] Carraro U, Rossini K, Mayr W, Kern H: Muscle fiber regeneration in human permanent lower motoneuron denervation: relevance to safety and effectiveness of FES-training, which induces muscle recovery in SCI subjects. Artif Organs 2005; 29: 187-191.

[42] Helgason T, Gargiulo P, Jóhannesdóttir F, Ingvarsson, $\mathrm{P}$, Knútsdóttir $\mathrm{S}$, Gudmundsdóttir V, Yngvason S: Monitoring Muscle Growth and Tissue Changes Induced by Electrical Stimulation of Denervated Degenerated Muscles with CT and Stereolithographic 3D Modeling. Artificial Organs 2005: 29; 440443.

[43] Kern H, Rossini K, Boncompagni S, Mayr W, Fanò G, Zanin ME, Podhorska-Okolow M, Protasi F, Carraro U: Long-term denervation in humans causes degeneration of both contractile and excitation-contraction coupling apparatus, which is reversible by functional electrical stimulation (FES): a role for myofiber regeneration? J Neuropath Exp Neurol 2004; 63: 919-931.
[44] Materialise company. Web site: http://www.materialise.com.

[45] Gargiulo P, Helgason T, Ingvarsson $P$, Knútsdóttir S, Gudmundsdóttir V, Yngvason $\mathrm{S}$. Morphological changes in denervated muscle treated with FES. Basic Appl Myol 2007; 17: 133-6

[46] Salmons S, Ashley Z, Sutherland H, Russold MF, Li F, Jarvis JC. Functional electrical stimulation of denervated muscles: basic issues. Artif Organs 2005;29:199-202.

[47] Silvia S. Blemker, Scott L. Delp. Rectus femoris and vastus intermedius fiber excursions predicted by three-dimensional muscle models. Journal of Biomechanics (2005).

[48] Szollar SM, Martin EME, Parthemore JG, Sartoris DJ, Deftos LJ, (1997) Demineralization in tetraplegic and paraplegic man over time, Spinal Cord, 35:223-228.

[49] Biering-Sørensen F, Bohr HH, Schaadt OP, (1990) Longitudinal study of bone mineral content in the lumbar spine, the forearm and the lower extremities after spinal cord injury, Europ J Clin Invest, 20:330-335.

[50] Wilmet E, Ismail AA, Heilporn A, Welraeds D, Bergmann P, (1995) Longitudinal study of the bone mineral content and of soft tissue composition after spinal cord section, Paraplegia, 33:674-677.

[51] Bauman WA, Spungen AM, Wang J, Pierson RNJr, Schwartz E, (1999) Continuous loss of bone during chronic immobilization: a monozygotic twin study, Osteoporos Int, 10:123-127.

[52] Chantraine A, Nusgens B, Lapiere CM, (1986) Bone remodeling during the development of osteoporosis in paraplegia, Calcif Tissue Int, 38:323-327.

[53] Finsen V, Indredavik B, Fougner KJ, (1992) Bone mineral and hormone status in paraplegics, Paraplegia, 30:343-347.

[54] www.beinvernd.is

[55] Kaplan PE, Roden W, Gilbert E, et al. Reduction of hypercalciuria in tetraplegia after weight-bearing and strengthening exercises. Paraplegia. 1981;19(5):289-93.

[56] Reiter AL, Volk A, Vollmar J, et al. Changes of basic bone turnover parameters in short-term and long-term patients with spinal cord injury. Eur Spine J. Jun 2007;16(6):771-6

[57] Garland DE, Adkins RH, Kushwaha V, et al. Risk factors for osteoporosis at the knee in the spinal cord injury population. J Spinal Cord Med. 2004;27(3):202-6 


\section{D Modelling and monitoring of denervated muscle under FES}

European Journal Translational Myology - Basic Applied Myology 2011; 21: 31-94

[58] Yilmaz B, Yasar E, Goktepe AS, et al. The relationship between basal metabolic rate and femur bone mineral density in men with traumatic spinal cord injury. Arch Phys Med Rehabil. Jun 2007;88(6):758-61

[59] Lind M, Bunger C. Factors stimulating bone formation. Eur Spine J 2001; 10 (Suppl 2): S102-S109 Rubin CT, McLeod KJ. Inhibition of osteopenia by biophysical intervention. In: Marcus R, Feldman D, Kelsey J (Editors), Osteoporosis.San Francisco: Academic Press; 1996.

[60] Hangartner TN, Rodgers MM, Glaser RM, Barre PS. Tibial bone density loss in spinal cord injured patients: effects of FES exercise. J Rehab Res Dev 1994; 31: 50-61.

[61] De Bell KK, Scremin AM, Perell KL, Kunkel CF. Effects of functional electrical stimulationinduced lower extremity cycling on bone density of spinal cord-injured patients. Am J Phys Med Rehab 1996; 75: 29-34

[62] Eser P, de Bruin ED, Telley I, Lechner HE, Knecht H,Stussi E. Effect of electrical stimulation-induced cycling on bone mineral density in spinal cord injured patients. Eur J Clin Invest 2003; 33: 412-419

[63] Dr. Carl T. Brighton, Gregory T. Tadduni, Stephen R. Goll, Solomon R. Treatment of denervation/disuse osteoporosis in the rat with a capacitively coupled electrical signal: Effects on bone formation and bone resorption Pollack 2 Journal of Orthopaedic Research 2005, Volume 6 Issue 5, Pages $676-684$.

[64] Young-hee Lee, Jung-ho Rah, Roh-wook Park and Chang-il Park. The effect of early therapeutic electrical stimulation on bone mineral density in the paralyzed limbs of the rabbit. Yonsei Medical Journal Vol. 42. No.2, pp 194-198, 2001.

[65] Bedell K. Effects of functional electrical stimulation-induced lower extremity cycling on bone density of spinal cord-injured patients ; American journal of physical medicine \& rehabilitation 1996, vol. 75, no1, pp. 29-34p

[66] Shih-Ching Chen. Increases in bone mineral density after functional electrical stimulation cycling exercises in spinal cord injured patients, Disability \& Rehabilitation, Volume 27, Issue 222005 , pages $1337-1341$.
[67] De Bell KK, Scremin AM, Perell KL, Kunkel CF. Effects of functional electrical stimulationinduced lower extremity cycling on bone density of spinal cord-injured patients. Am J Phys Med Rehab 1996; 75: 29-34.

[68] Mohr T, Podenphant J, Biering-Sorensen F, Galbo H, Thamsborg G, Kjaer M. Increased bone mineral density after prolonged electrically induced cycle training of paralysed limbs in spinal cord injured man. Calcif Tiss Int 1997; 61: 22-25.

[69] Eser P, de Bruin ED, Telley I, Lechner HE, Knecht H, Stussi E. Effect of electrical stimulation-induced cycling on bone mineral density in spinal cord injured patients. Eur J Clin Invest 2003; 33: 412-419

[70] JM Clark. Physiological effects of lower extremity functional electrical stimulation in early spinal cord injury: lack of efficacy to prevent bone loss. Spinal Cord (2007) 45, 78 85.

[71] T. Helgason, P. Gargiulo, P. Ingvarsson, S. Yngvason Comparision of femur bone density, muscle size and mechanical proprieties by denervated and degenerated thigh muscles" 9th Vienna International workshop on FES, September 2007, Vienna Austria p.46-49

[72] T.Helgason, P.Gargiulo, P. Ingvarsson, S. Yngvason "Comparing Muscle and Bone Density changes in Denervated and Degenrated Muscle Treated with Electrical Stimulation" 13th Annual International FES Society Conference, September 2008, Freiburg Germany p. 259-261

[73] Fjóla Jóhannesdóttir. BONE - Use it or lose it. Master thesis, University of Iceland 2006

[74] K. Donina and A. Yarusskaya. "Density of Bone," The Physics. Factbook, Ed. Glenn Elert, 2002

[75] Genant HK, Faulkner KG, Glfier CC. Measurement of bone mineral density: current status. Am J Med 1991;91:(Suppl 5B):49-53.

[76] Faulkner KG, Glüer CC, Grampp S, Genant HK. Cross-calibration of liquid and solid QCT calibration standards: corrections to the UCSF normative data. Osteoporos Int. 1993 Jan;3(1):36-42

[77] Cowin Stephan C. Bone Mechanics Handbook. CRC Press, Boca Raton, Florida, 2th edition, 2001.1. 


\section{D Modelling and monitoring of denervated muscle under FES}

European Journal Translational Myology - Basic Applied Myology 2011; 21: 31-94

\section{Appendix}

\section{Curvature ratio}

Table A : Curvature values, mean $\mathrm{C}$ values and ratio results for Patient 1 . Red numbers represents an increase in $\mathrm{C}$, black a decrease in $\mathrm{C}$ and green represents no change in curvature

Patient : 1

\begin{tabular}{|c|c|c|c|c|c|c|c|c|c|c|c|c|}
\hline \multirow[t]{2}{*}{ Segm. length [mm] } & \multicolumn{2}{|c|}{8,54} & \multicolumn{2}{|c|}{8,95} & \multicolumn{2}{|c|}{8,33} & \multicolumn{2}{|c|}{8,13} & \multicolumn{2}{|c|}{7,93} & \multicolumn{2}{|c|}{8,16} \\
\hline & 2003 & $03 / 03$ & 2004 & $04 / 03$ & 2005 & $05 / 03$ & 2006 & $06 / 03$ & 2007 & $07 / 03$ & 2008 & $08 / 03$ \\
\hline \multirow[t]{5}{*}{$\mathrm{C}$ values $[\mathrm{mm}-1]$ : } & \multicolumn{2}{|c|}{0,58} & \multicolumn{2}{|c|}{0,43} & \multicolumn{2}{|c|}{0,92} & \multicolumn{2}{|c|}{0,67} & \multicolumn{2}{|c|}{0,56} & \multicolumn{2}{|c|}{2,17} \\
\hline & \multicolumn{2}{|c|}{0,46} & \multicolumn{2}{|c|}{0,78} & \multicolumn{2}{|c|}{0,60} & \multicolumn{2}{|c|}{0,75} & & & & 60 \\
\hline & & & & & & 82 & & 30 & & & & 80 \\
\hline & & & & & & 24 & & 13 & & 46 & & 15 \\
\hline & & & & & & 43 & & 38 & & & & 26 \\
\hline Average: & 0,46 & 1,00 & 0,51 & 1,11 & 0,60 & 1,30 & 0,45 & 0,98 & 0,59 & 1,28 & 0,80 & 1,74 \\
\hline & & & & & & 98 & & & & & & 41 \\
\hline & & & & & & 51 & & 33 & & & & 79 \\
\hline & & & & & & 16 & & 27 & & 25 & & 49 \\
\hline & & & & & & 18 & & 13 & & & & 79 \\
\hline & & & & & & 23 & & 13 & & & & 21 \\
\hline & 0,30 & 1,00 & 0,21 & 0,70 & 0,41 & 1,37 & 0,23 & 0,77 & 0,44 & 1,47 & 0,54 & 1,80 \\
\hline & & & & & & 23 & & 43 & & & & 05 \\
\hline & & & & & & 05 & & 40 & & & & 04 \\
\hline & & & & & & 18 & & 10 & & & & 58 \\
\hline & & & & & & 52 & & 10 & & & & 67 \\
\hline & & & & & & 18 & & 05 & & & & 10 \\
\hline & 0,09 & 1,00 & 0,41 & 4,56 & 0,23 & 2,56 & 0,22 & 2,44 & 0,20 & 2,22 & 0,29 & 3,22 \\
\hline & & & & & & 23 & & 03 & & & & 71 \\
\hline & & & & & & 17 & & 08 & & & & 44 \\
\hline & & & & & & 75 & & 56 & & & & 02 \\
\hline & & & & & & 06 & & 08 & & & & 10 \\
\hline & & & & & & 11 & & 27 & & & & 07 \\
\hline & 0,29 & 1,00 & 0,09 & 0,31 & 0,26 & 0,90 & 0,20 & 0,69 & 0,16 & 0,55 & 0,27 & 0,93 \\
\hline & & & & & & 79 & & 33 & & & & 63 \\
\hline & & & & & & 31 & & 24 & & & & 52 \\
\hline & & & & & & 05 & & 56 & & & & 32 \\
\hline & & & & & & 09 & & 38 & & & & 39 \\
\hline & & & & & & 06 & & 68 & & & & 37 \\
\hline & 0,06 & 1,00 & 0,27 & 4,50 & 0,26 & 4,33 & 0,44 & 7,33 & 0,19 & 3,17 & 0,45 & 7,50 \\
\hline & & & & & & 01 & & 13 & & & & 01 \\
\hline & & & & & & 06 & & 08 & & & & 06 \\
\hline & & & & & & 17 & & 13 & & & & 14 \\
\hline & & & & & & 81 & & 07 & & & & 06 \\
\hline & & & & & & 22 & & & & & & 11 \\
\hline & 0,04 & 1,00 & 0,23 & 5,75 & 0,25 & 6,25 & 0,10 & 2,50 & 0,14 & 3,50 & 0,08 & 2,00 \\
\hline & & & & & & 05 & & 01 & & & & 10 \\
\hline & & & & & & 01 & & 54 & & & & 12 \\
\hline & & & & & & 01 & & 49 & & & & 09 \\
\hline & & & & & & 10 & & 31 & & & & 15 \\
\hline & & & & & & 17 & & 15 & & & & 12 \\
\hline & 0,31 & 1,00 & 0,18 & 0,58 & 0,07 & 0,23 & 0,30 & 0,97 & 0,06 & 0,19 & 0,12 & 0,39 \\
\hline & & & & & & 02 & & 09 & & & & 04 \\
\hline & & & & & & 16 & & 09 & & 28 & & 17 \\
\hline & & & & & & 05 & & 20 & & & & 08 \\
\hline & & & & & & 03 & & 07 & & 01 & & 03 \\
\hline & & & & & & 19 & & 12 & & & & 11 \\
\hline & 0.30 & 1.00 & 0.43 & 1.43 & 0.09 & 0.30 & 0.11 & 0.37 & 0.11 & 0.37 & 0.09 & 0.30 \\
\hline
\end{tabular}


3D Modelling and monitoring of denervated muscle under FES

European Journal Translational Myology - Basic Applied Myology 2011; 21: 31-94

\begin{tabular}{|c|c|c|c|c|c|c|c|c|c|c|c|}
\hline & & & & & & \multicolumn{2}{|c|}{0,05} & \multicolumn{2}{|c|}{0,15} & \multicolumn{2}{|c|}{0,05} \\
\hline & & & & & & \multicolumn{2}{|c|}{0,45} & \multicolumn{2}{|c|}{0,04} & \multicolumn{2}{|c|}{0,01} \\
\hline & & & & & & \multicolumn{2}{|c|}{0,29} & \multicolumn{2}{|c|}{0,03} & \multicolumn{2}{|c|}{0,27} \\
\hline & & & & & & \multirow{2}{*}{\multicolumn{2}{|c|}{$\begin{array}{l}0,12 \\
0,58\end{array}$}} & \multirow{2}{*}{\multicolumn{2}{|c|}{$\begin{array}{l}0,13 \\
0,27\end{array}$}} & \multicolumn{2}{|c|}{0,13} \\
\hline \multicolumn{2}{|c|}{0,09} & \multicolumn{2}{|c|}{0,57} & & & & & & & \multicolumn{2}{|c|}{0,18} \\
\hline 0,50 & 1,00 & 0,60 & 1,20 & 0,33 & 0,66 & 0,30 & 0,60 & 0,12 & 0,24 & 0,13 & 0,26 \\
\hline \multicolumn{2}{|c|}{0,71} & \multicolumn{2}{|c|}{0,59} & \multicolumn{2}{|c|}{0,38} & \multicolumn{2}{|c|}{0,35} & \multicolumn{2}{|c|}{0,68} & \multicolumn{2}{|c|}{0,68} \\
\hline \multicolumn{2}{|c|}{0,08} & \multicolumn{2}{|c|}{0,13} & & & \multicolumn{2}{|c|}{0,60} & \multicolumn{2}{|c|}{0,83} & \multicolumn{2}{|c|}{0,94} \\
\hline \multicolumn{2}{|c|}{0,20} & \multicolumn{2}{|c|}{0,39} & & & \multicolumn{2}{|c|}{0,58} & \multicolumn{2}{|c|}{0,74} & \multicolumn{2}{|c|}{0,22} \\
\hline \multicolumn{2}{|c|}{0,08} & \multicolumn{2}{|c|}{0,40} & & & \multicolumn{2}{|c|}{1,27} & \multicolumn{2}{|c|}{0,67} & \multicolumn{2}{|c|}{0,24} \\
\hline \multicolumn{2}{|c|}{0,18} & & & & & & & & & & \\
\hline 0,25 & 1,00 & 0,41 & 1,64 & 0,44 & 1,76 & 0,80 & 3,20 & 0,64 & 2,56 & 0,46 & 1,84 \\
\hline ). & & & & Pat & & & & & & & \\
\hline
\end{tabular}

Table B : Curvature values, mean C values and ratio results for Patient 2.

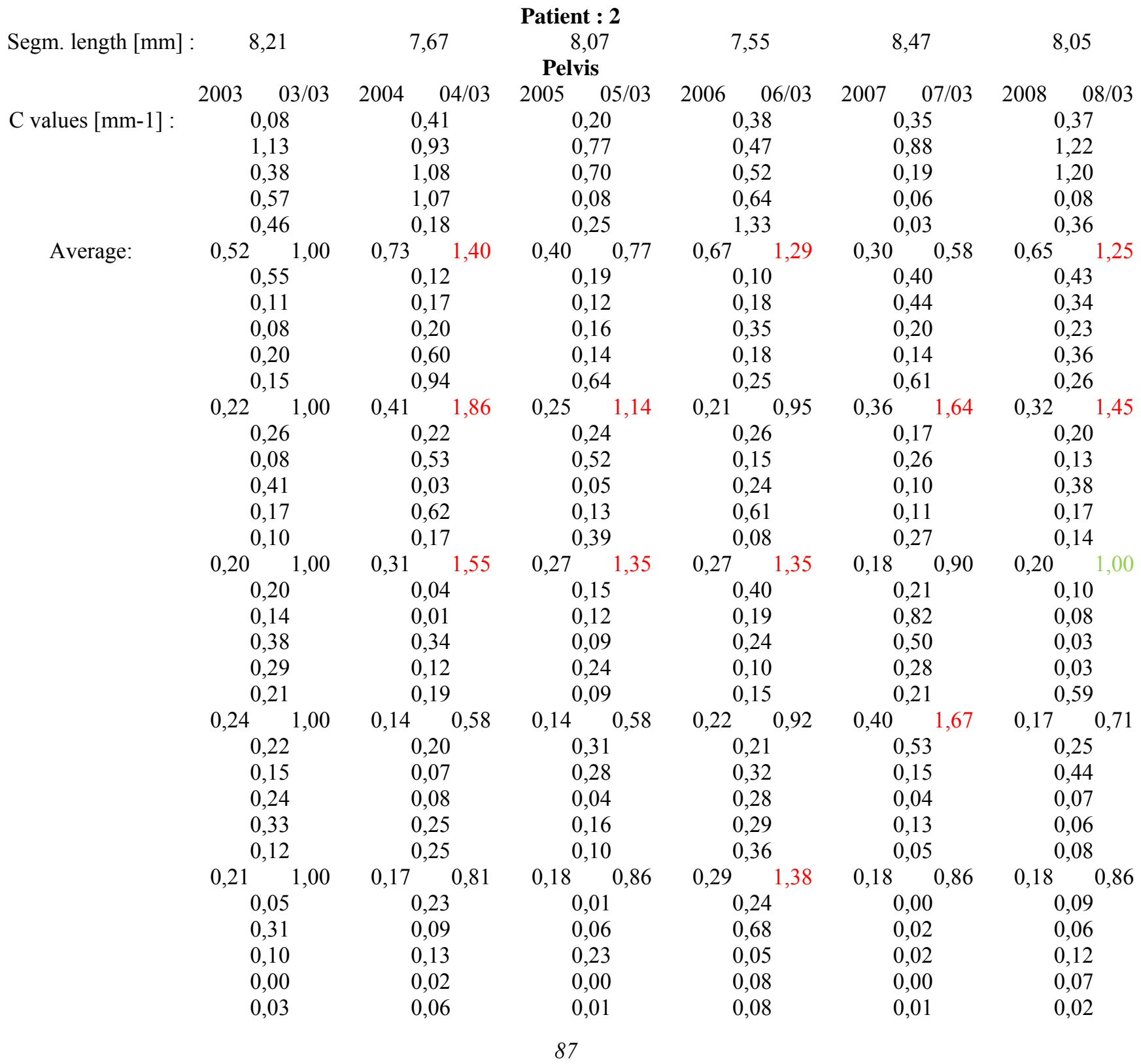


3D Modelling and monitoring of denervated muscle under FES

European Journal Translational Myology - Basic Applied Myology 2011; 21: 31-94

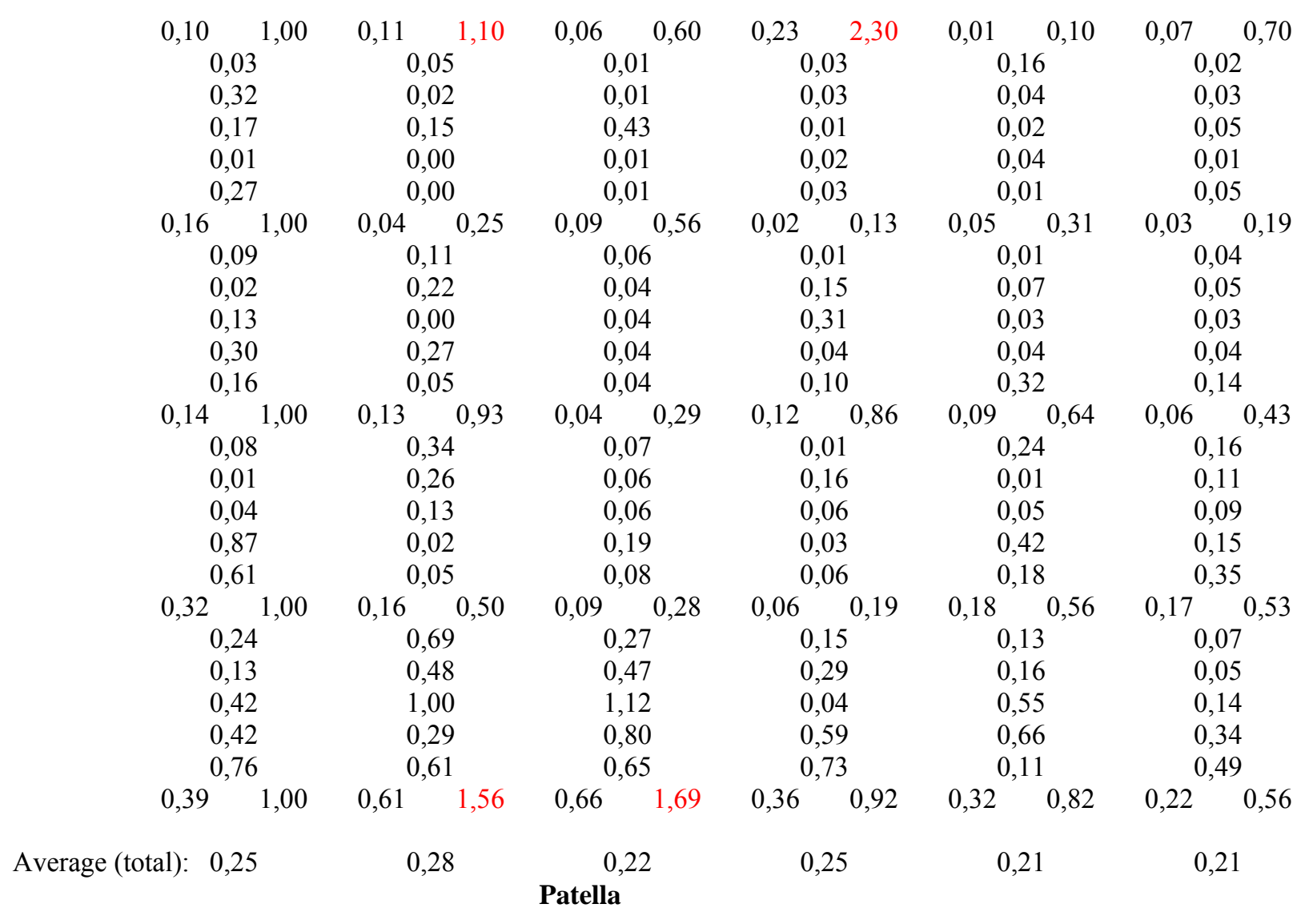


Table C: Curvature values, mean C values and ratio results for Patient 3.

\begin{tabular}{|c|c|c|c|c|c|c|c|c|c|c|}
\hline \multirow{2}{*}{ Segm. length [mm] } & \multicolumn{6}{|c|}{ Patient : 3} & \multirow{2}{*}{\multicolumn{2}{|c|}{8,42}} & \multirow{2}{*}{\multicolumn{2}{|c|}{8,41}} \\
\hline & \multicolumn{2}{|c|}{8,51} & $\begin{array}{r}8 \\
\text { Pel }\end{array}$ & & \multicolumn{2}{|c|}{8,28} & & & & \\
\hline & 2003 & 03/03 & 2004 & $04 / 03$ & 2005 & $05 / 03$ & 2006 & $06 / 03$ & 2007 & $07 / 03$ \\
\hline \multirow{5}{*}{$\mathrm{C}$ values $[\mathrm{mm}-1]:$} & \multicolumn{2}{|c|}{0,74} & \multicolumn{2}{|c|}{1,12} & \multicolumn{2}{|c|}{0,60} & \multicolumn{2}{|c|}{1,19} & \multicolumn{2}{|c|}{0,31} \\
\hline & \multicolumn{2}{|c|}{0,35} & \multicolumn{2}{|c|}{0,64} & \multicolumn{2}{|c|}{2,54} & \multicolumn{2}{|c|}{0,89} & & 39 \\
\hline & & 23 & & & & & & & & 30 \\
\hline & & ,44 & & & & & & & & 27 \\
\hline & & 16 & & & & & & & & 48 \\
\hline Average: & 0,38 & 1,00 & 0,62 & 1,63 & 1,00 & 2,63 & 0,55 & 1,45 & 0,55 & 1,45 \\
\hline & &, 30 & & & & & & & & 38 \\
\hline & & 07 & & & & & & & & 46 \\
\hline & &, 53 & & & & & & & & 30 \\
\hline & & 09 & & & & & & & & 32 \\
\hline & & 37 & & & & & & & & 77 \\
\hline & 0,27 & 1,00 & 0,30 & 1,11 & 0,43 & 1,59 & 0,28 & 1,04 & 0,45 & 1,67 \\
\hline & &, 12 & & & & & & & & 28 \\
\hline & & 39 & & & & & & & & 00 \\
\hline & & 42 & & & & & & & & 05 \\
\hline & & 07 & & & & & & & & 48 \\
\hline & & 30 & & & & & & & & 20 \\
\hline & 0,26 & 1,00 & 0,25 & 0,96 & 0,27 & 1,04 & 0,42 & 1,62 & 0,20 & 0,77 \\
\hline & &, 44 & & & & & & & & 11 \\
\hline & & 13 & & & & & & & & 18 \\
\hline & & 04 & & & & & & & & 51 \\
\hline & & 03 & & & & & & & & 15 \\
\hline & & 07 & & & & & & & & 36 \\
\hline & 0,14 & 1,00 & 0,17 & 1,21 & 0,12 & 0,86 & 0,16 & 1,14 & 0,26 & 1,86 \\
\hline & &, 15 & & & & & & & & 11 \\
\hline & & 14 & & & & & & & & 04 \\
\hline & & 03 & & & & & & & & 19 \\
\hline & & 04 & & & & & & & & 09 \\
\hline & & 10 & & & & & & & & 56 \\
\hline & 0,09 & 1,00 & 0,24 & 2,67 & 0,11 & 1,22 & 0,21 & 2,33 & 0,20 & 2,22 \\
\hline & &, 10 & & & & & & & & 27 \\
\hline & & 01 & & & & & & & & 16 \\
\hline & &, 02 & & & & & & & & 06 \\
\hline & & 12 & & & & & & & & 01 \\
\hline & & 01 & & & & & & & & 08 \\
\hline & 0,05 & 1,00 & 0,16 & 3,20 & 0,11 & 2,20 & 0,05 & 1,00 & 0,12 & 2,40 \\
\hline & & ,03 & & & & & & & & 07 \\
\hline & & 14 & & & & & & & & 09 \\
\hline & & 07 & & & & & & & & 10 \\
\hline & & 09 & & & & & & & & 03 \\
\hline & &, 07 & & & & & & & & 03 \\
\hline & 0,08 & 1,00 & 0,05 & 0,63 & 0,18 & 2,25 & 0,08 & 1,00 & 0,06 & 0,75 \\
\hline & &, 15 & & & & & & & & 01 \\
\hline & & 05 & & & & & & & & 10 \\
\hline & & 10 & & & & & & & & 02 \\
\hline & & 06 & & & & & & & & 17 \\
\hline & & 28 & & & & & & & & 02 \\
\hline & 0,13 & 1,00 & 0,08 & 0,62 & 0,10 & 0,77 & 0,13 & 1,00 & 0,06 & 0,46 \\
\hline & &, 60 & & & & & & & & 01 \\
\hline & & 10 & & & & & & & & 08 \\
\hline & & 35 & & & & & & & & 30 \\
\hline & & 47 & & & & & & & & 07 \\
\hline
\end{tabular}


3D Modelling and monitoring of denervated muscle under FES

European Journal Translational Myology - Basic Applied Myology 2011; 21: 31-94

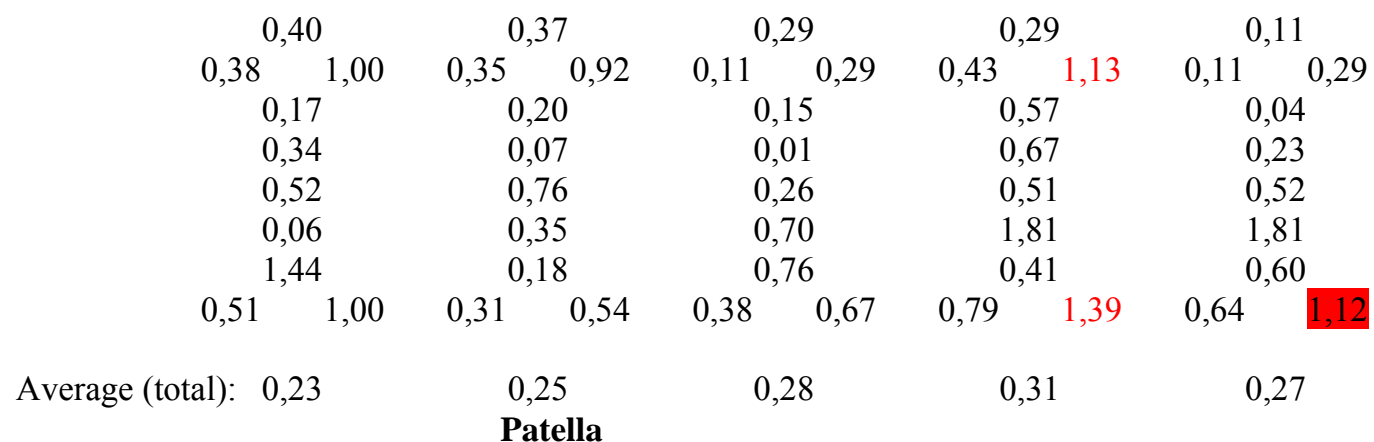

\section{BMD Conversion and visualization. (Matlab script)}

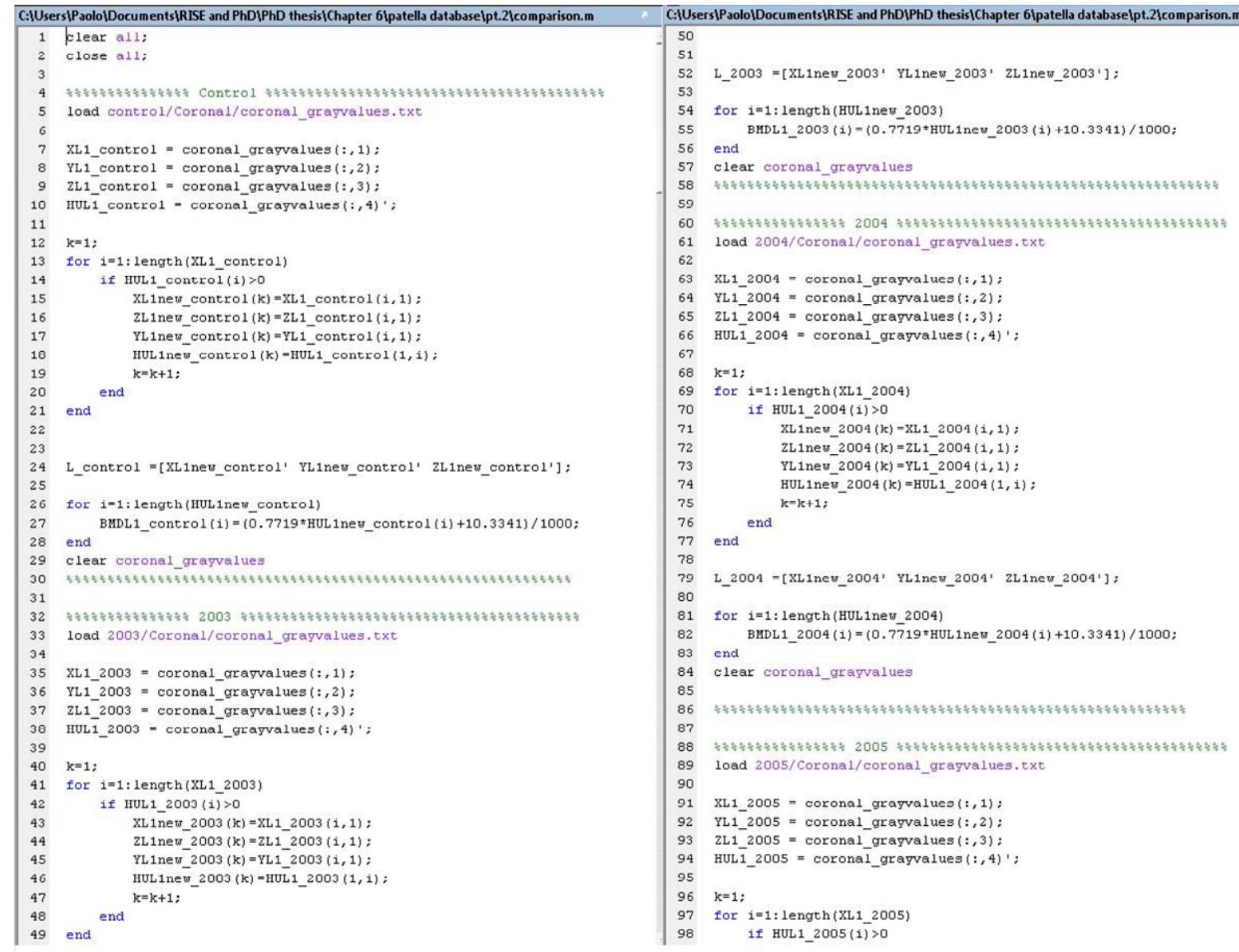


3D Modelling and monitoring of denervated muscle under FES

European Journal Translational Myology - Basic Applied Myology 2011; 21: 31-94
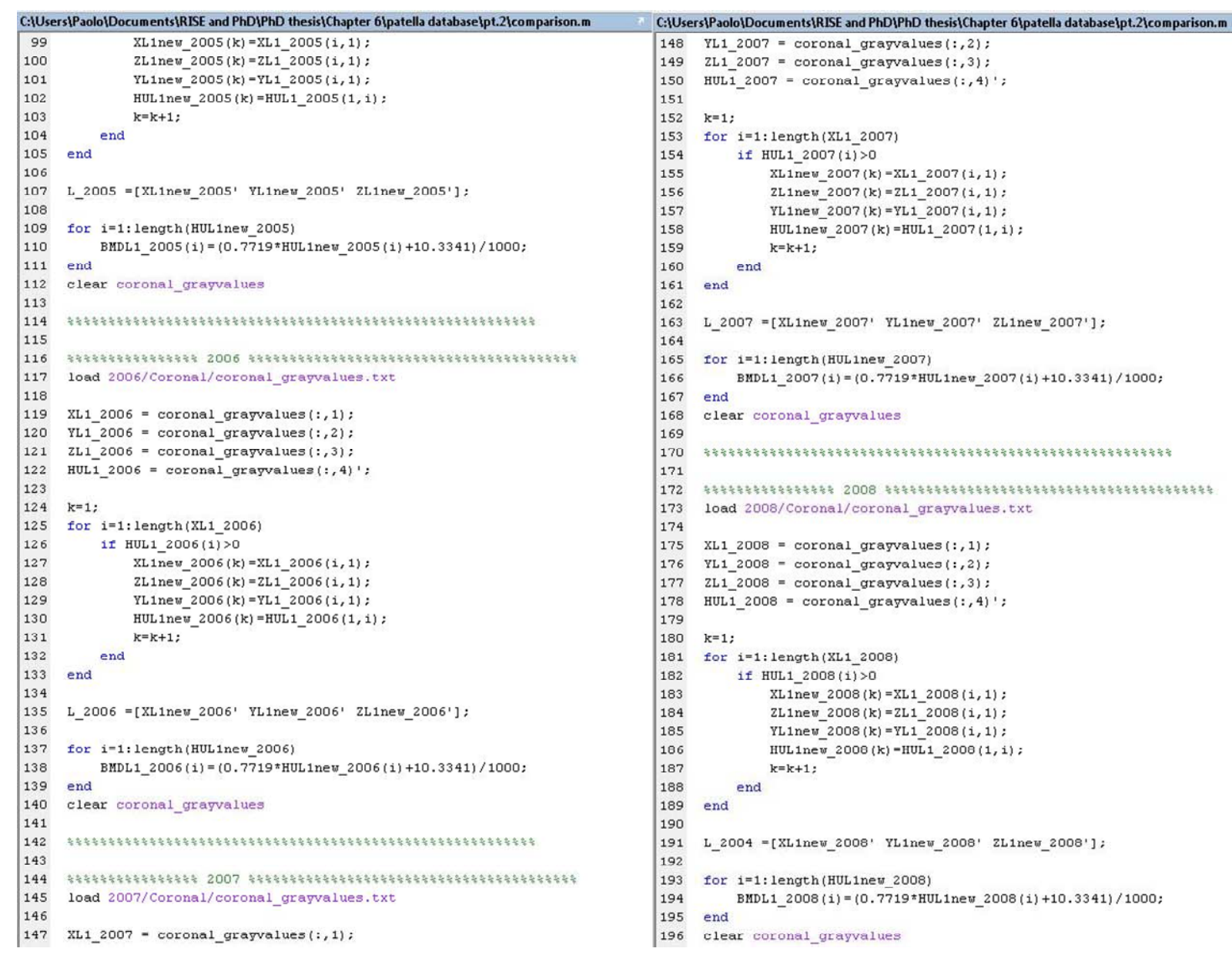


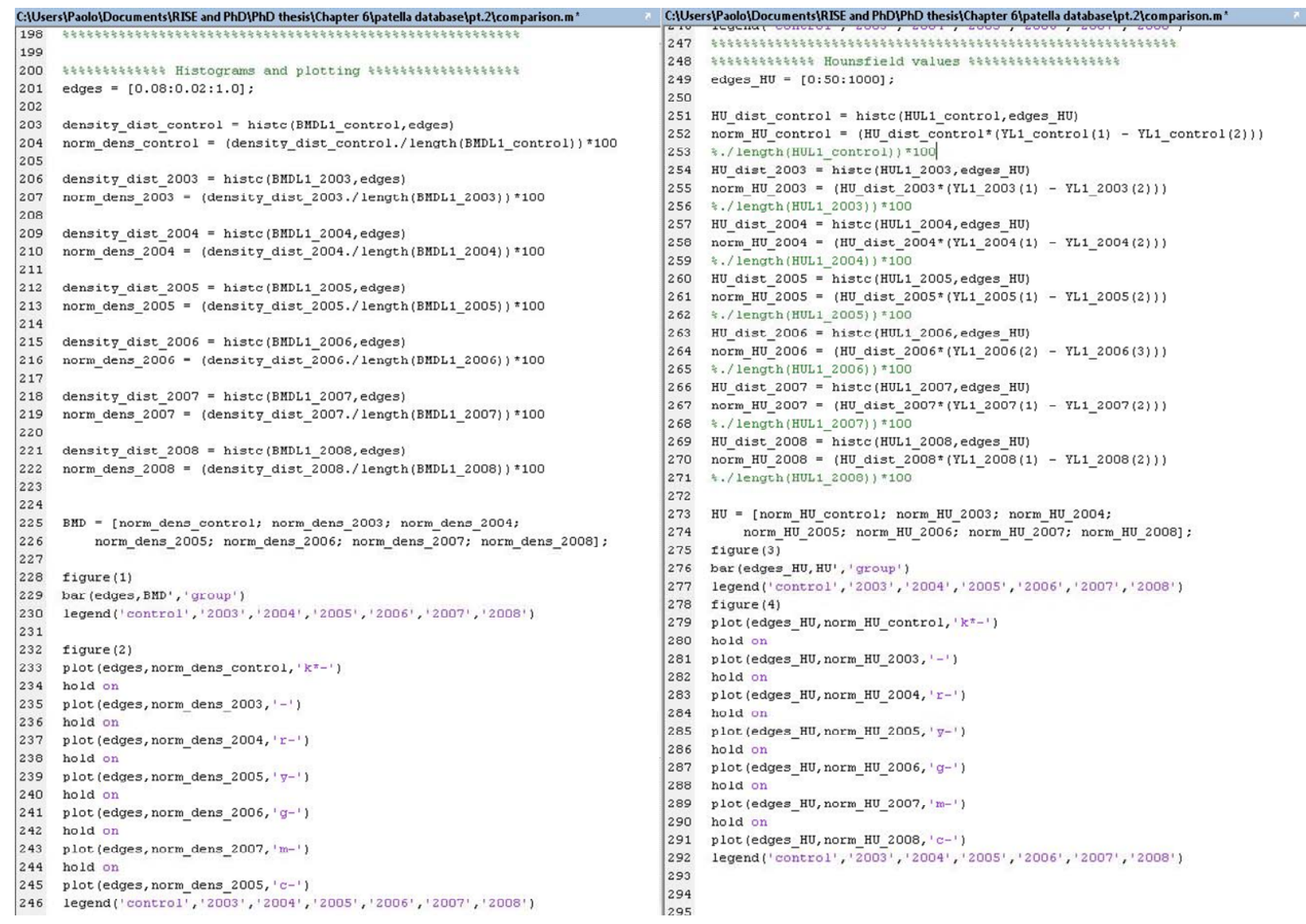

\section{Curriculum Vitae}

\section{Contact info:}

Name: Paolo Gargiulo

Id: $190573-2149$

Home Address: Vörðurstig 3, 220 Hafnarfjörður

Work Address: Department of Research and Development, Landspitali - University Hospital, LSH , Ármuli 1a, 105

Reykjavik,

E-mail: paologar@landspitali.is.

Phone Gsm: 8245384, Office: 5431533, Home: 5552919

\section{Main career events:}

19-05-1973 Born in Naples- Italy from Ciro Gargiulo and Elvira Primativo

1987 - 1992 Qualified at the Technical institute of informatics and electronic "F. Giordani" Naples, Italy

1992 - 2001 Graduated at the University of Naples "Federico II" Degree in Electronic engineers and Master in

Biomedical engineering

1999 - 2001 Master thesis at Research and Development department, University Hospital Iceland: "Research and experimentation of telemedicine applications for seamen in the North Atlantic"

Since 01/2002 Full position as Biomedical Engineer at Research and Development Department, Landspitali -

University Hospital of Iceland, LHS.

2002-2003 Design and development of the Icelandic Pacemaker database

Research project: The Role of longevity in the purchase of Pacemaker based on Icelandic Implant Statistics

2003-2004 Development project: Tele ECG: ECG recorder and transmission by Bluetooth 
2005-2006 Lecturer at the University of Reykjavik for the following courses:

Winter 2005 Lífupplýsingatækni for students of Tækniháskóli Íslands.

Autumn 2006 Physic Laboratory for the engineer students

10/2005 Computational work and modelling in the Icelandic part of the European project RISE. The RISE project is a part of the $5^{\text {th }}$ framework of the EU. The aim of the project is to develop a new rehabilitation method based on electrical stimulation for treatment of flaccid paraplegia.

09-10/06 Research work on modelling denervated muscle treated with FES at Centre for Biomedical Engineering and Physic Medical University of Vienna

10/2006 Registered at the Vienna University of Technology, TU Wien as PhD Student

06/2007 Grant from Rannis Icelandic research fund, to support the PhD work

07/2007 Co-author in the winning paper at Annual International Conference on Computer Guided Implantology and Advanced 3D Medical Image Processing Washington 1-3 June 2007: "Using Mimics to Monitor Changes in Bone Mineral Density of Femur during Electrical Stimulation Therapy of Denervated Degenerated Thigh Muscles" 01/2008 Teaching at biomedical engineering department - University of Reykjavik, the course "Artificial Organs and Prosthesis".

\section{Articles in peer-reviewed scholarly journals}

P. Gargiulo , B. Vatnsdal, P. Ingvarsson, S.Knútsdóttir, V. Gudmundsdóttir, S.Yngvason and T. Helgason.. Restoration of Muscle Volume and Shape Induced by Electrical Stimulation of Denervated Degenerated Muscles: Qualitative and Quantitative Measurement of Changes in Rectus Femoris Using Computer Tomography and Image Segmentation. Artificial Organs (2008) 32(8):609-613.

P. Gargiulo, T. Helgason, P. Ingvarsson, S. Knútsdóttir, V. Gudmundsdóttir, and S. Yngvason "Morphological changes in Rectus Femoris Muscle: Advanced Image Processing Technique and 3-Dimensional Visualization to Monitor Denervated and Degenerated Muscles Treated with Functional Electrical Stimulation.” Journal of Basic and Applied Myology, BAM 17 (3\&4):133-136, 2007

T. Helgason, P. Gargiulo, F. Jóhannesdóttir, P. Ingvarsson, S. Knútsdóttir, V. Gudmundsdóttir, S. Yngvason. "Monitoring muscle growth and tissue changes induced by electrical stimulation of denervated degenerated muscles with CT Stereolithographic 3-D modelling" Artificial Organs, Vol 29, Issue 6, pp. 440-443

\section{Articles, posters and abstracts in peer-reviewed conferences/ symposia/ proceedings}

P. Gargiulo, T. Helgason, P. Ingvarsson, S. Yngvason "Denervated and degenerated muscle stimulation: advanced image processing techniques and 3-dimensional rendering to monitor muscles restoration" 13th Annual International FES Society Conference, September 2008, Freiburg Germany p. 408-410

T. Helgason, P. Gargiulo, P. Ingvarsson, S. Yngvason "Comparing Muscle and Bone Density changes in Denervated and Degenrated Muscle Treated with Electrical Stimulation" 13th Annual International FES Society Conference, September 2008, Freiburg Germany p. 259-261

P. Gargiulo, T.Helgason, P. Ingvarsson, S. Yngvason "Denervated Degenerated Muscle growth influenced by FES: A novel monitoring methodology based on muscle segmentation " 9th Vienna International workshop on FES, September 2007, Vienna Austria p.50-54

T. Helgason, P. Gargiulo, P. Ingvarsson, S. Yngvason " Comparision of femur bone density, muscle size and mechanical proprieties by denervated and degenerated thigh muscles" 9th Vienna International workshop on FES, September 2007, Vienna Austria p.46-49

P. Gargiulo, T. Helgason P. Ingvarsson, S. Yngvason "Segmentation of muscle bellies: Monitoring of denervated degenerated muscle growth influenced by FES" $46^{\mathrm{TH}}$ Annual Scientific Meeting of ISCoS $10^{\text {th }}$ Biannual Congress of NoSCoS 27 - 30 June 2007, Reykjavík, Iceland. ISCoS- NoSCoS Proceeding p. 219

P. Gargiulo, T. Helgason, P. Ingvarsson, S. Yngvason " Segmentation of muscle bellies using MIMICS" MIMICS Innovation Awards 2007 "Annual International Conference on Computer Guided Implantology and Advanced 3D Medical Image Processing” Washington 1-3 June 2007. 


\section{D Modelling and monitoring of denervated muscle under FES}

European Journal Translational Myology - Basic Applied Myology 2011; 21: 31-94

T. Helgason, P. Gargiulo, P. Ingvarsson, S. Yngvason "Using Mimics to Monitor Changes in Bone Mineral Density of Femur during Electrical Stimulation Therapy of Denervated Degenerated Thigh Muscles" "Annual International Conference on Computer Guided Implantology and Advanced 3D Medical Image Processing" Washington 1-3 June 2007

Helgason T., Gargiulo P., Helgason K., Arnar D.O., Gottskalksson G. "A Pacemaker and ICD Register in Iceland“" IFMBE proceedings, Vol. 6, 2004.

Gargiulo, P.; Valdimarsdottir, M.; Palsson, T.; Helgason, T.: "Telemidicine in Iceland: Current Status, Challenges and Prospective“"Proceedings of Medicom and Health Telematics 2004, Ischia- Naples, Italy, Article nr. 363.

Helgason, T.; Gargiulo, P, Jóhannesdottir, F. Ingvarsson, P. Knutsdottir, S.; Gudmundsdottir, V.; Yngvason, S.: „Applications of Stereolithographie in Monitoring Muscle Growth Induced by Electrical Stimulation of Denervated Degenerated Muscles“, 8th Vienna International Workshop on Functional Electrical Stimulation, Proceedings, Austrian Society of Biomedical Engineering, 2004, p. 241-242.

Gargiulo, P.; Helgason, T.; Bifulco, P.; Bracale, M.: „Telemedicine applications for seamen in the North Atlantc",Proceedings of the International Federation for Medical \& Biological Engineering, Vol. 2, 2002, s.: 250-1.

Helgason, T.; Gargiulo, P.; Weihe, P.; Kristinsson, S.; Björgvinsson, JB.; Pálsson, T.; Jespersen, J.; „Telemedicine for the seafarer in the North Atlantic“", Nortelemed, $4^{\text {th }}$ Nordic Congress on Telemedicine/Norsk Telemed 2002, s.: 117. 